Portland State University

PDXScholar

Spring 5-26-2016

\title{
Event Detection Using Correlation within Arrays of Streaming PMU Data
}

Jordan Landford

Portland State University

Follow this and additional works at: https://pdxscholar.library.pdx.edu/open_access_etds

Part of the Power and Energy Commons

Let us know how access to this document benefits you.

\section{Recommended Citation}

Landford, Jordan, "Event Detection Using Correlation within Arrays of Streaming PMU Data" (2016). Dissertations and Theses. Paper 3031.

https://doi.org/10.15760/etd.3026

This Thesis is brought to you for free and open access. It has been accepted for inclusion in Dissertations and Theses by an authorized administrator of PDXScholar. Please contact us if we can make this document more accessible: pdxscholar@pdx.edu. 
Jordan Landford

A thesis submitted in partial fulfillment of the requirements for the degree of

\author{
Master of Science \\ in \\ Electrical and Computer Engineering
}

Thesis Committee:

Robert Bass, Chair

Melinda Holtzman

Jonathan Bird

Portland State University

2016 
(C) 2016 Jordan Landford 


\section{Abstract}

This thesis provides a synchrophasor data analysis methodology that leverages both statistical correlation techniques and a statistical distribution in order to identify data inconsistencies, as well as power system contingencies. This research utilizes archived Phasor Measurement Unit (PMU) data obtained from the Bonneville Power Administration in order to show that this methodology is not only feasible, but extremely useful for power systems monitoring, decision support, and planning purposes.

By analyzing positive sequence voltage angles between a pair of PMUs at two different substation locations, an historic record of correlation is established. From this record, a Rayleigh distribution of correlation coefficients is calculated. The statistical parameters of this Rayleigh distribution are used to infer occurrences of power system and data events.

To monitor an entire system, a simple solution would be observing each of these parameters for every PMU combination. One issue with this approach is that correlation of some PMU pairs may be redundant or yield little value to monitoring capabilities. Additionally, this approach quickly encounters scalability issues as each additional PMU adds considerably to computation - for example, if the system contains $n$ PMUs the amount of computations will be $\frac{n(n-1)}{2}$. System-wide monitoring of these parameters in this fashion is cumbersome and inefficient. 
To address these issues, an alternative scheme is proposed which involves monitoring only a subset of PMUs characterized by electrically coupled zones, or clusters, of PMUs. These clusters include both electrically-distant and electrically-near PMU sites. When monitored over an event, these yield statistical parameters sufficient for detecting event occurrences. This clustering scheme can be utilized to significantly decrease computation time and allocation of resources while maintaining optimal system observability.

Results from the statistical methods are presented for a select few case studies for both data and power system event detection. In addition, determination of cluster size and content is discussed in detail. Lastly, the viability of monitoring pertinent statistical parameters over various clustering schemes is demonstrated. 


\section{Dedication}

Dedicated to those who have supported and fostered my academic pursuit. These include my mother and her boyfriend, George, for financially supporting me during a majority of my undergraduate years. My common law wife, Mary Grace, for providing me with emotional support, helping me grow as a person, and taking care of me during times of sickness. Lynn Montoya and Sarah Elsasser at the PCC ROOTS organization for helping me figure out a plan early in my academic career. Dr. Melinda Holtzman for playing a large role in assisting me in obtaining the ORBest scholarship. Lastly, my advisor, Dr. Robert Bass, for providing such a great opportunity through this research project, the ORBest scholarship, and through his fantastic power engineering program at the Maseeh College of Engineering and Computer Science. 


\section{Acknowledgements}

A deep and sincere thank you to Tony Ferris at the Bonneville Power Administration,

Johanna Brickman from Oregon BEST, and Rachel Stansbury from the Maseeh College. 


\section{Contents}

$\begin{array}{ll}\text { Abstract } & \text { i }\end{array}$

Dedication

Acknowledgements $\quad$ iv

List of Tables

List of Figures $\quad$ viii

1 Problem Statement 1

$2 \quad$ Literature Review 4

2.1 Intermediate Work . . . . . . . . . . . . . . . . . 7

3 Background 9

3.1 Synchrophasor IEEE Standard . . . . . . . . . . . . . . . . . . . 9 9

3.1.1 Phasor Definition . . . . . . . . . . . . . . . . 10

3.1.2 Timetags and Synchronization . . . . . . . . . . . . . 10

3.1.3 Measurement Requirements . . . . . . . . . . . . . . . . 11

3.2 Sequence Components . . . . . . . . . . . . . . . . . 12

3.3 Dataset Characteristics ....................... 14

3.4 Network Topology . . . . . . . . . . . . . . . . . 15

4 Design Methodology 17

4.1 Correlation . . . . . . . . . . . . . . . . 17

4.2 Window Length . . . . . . . . . . . . . . . . . . . . . . . . . . . . . . . .

4.3 Correlation Visual . . . . . . . . . . . . . . . . . . 20

4.3.1 Historic Playback Engine . . . . . . . . . . . . . . . 21

4.3.2 Data Structure . . . . . . . . . . . . . . . 22

4.4 Electrical Distance . . . . . . . . . . . . . . . . . . . 24

4.5 Rayleigh Distribution . . . . . . . . . . . . . . . 25

4.5.1 Event Detection Metric . . . . . . . . . . . . . . 30

4.6 Data Events . . . . . . . . . . . . . . . . . . . . . . . . . . . . . . . . .

4.7 Power System Events . . . . . . . . . . . . . . . . . . . . . 40 
4.8 Cluster Size . . . . . . . . . . . . . . . . . . . . . . . . . . . . 42

4.9 Cluster Content . . . . . . . . . . . . . . . . . . . . . 44

$5 \quad$ Results \& Analysis $\quad 47$

5.1 Detecting Data Errors and Power System Events . . . . . . . . . . . . 49

5.2 Window Length . . . . . . . . . . . . . . . . 53

5.3 Rayleigh Characteristics . . . . . . . . . . . . . . . . 58

5.4 Cluster Scheme . . . . . . . . . . . . . . . . . . . . . . . . . . . . . . . . . . . . . . .

5.4 Analyzing Other Events . . . . . . . . . . . 70

$\begin{array}{llr}6 & \text { Discussion } & 86\end{array}$

$\begin{array}{llr}7 & \text { Conclusion } & 89\end{array}$

$\begin{array}{ll}\text { Bibliography } & 91\end{array}$

Appendix A: Python / MATLAB Code Repository 93 


\section{List of Tables}

4.1 Negative $r$ entries (in percent) of $\phi_{+} \ldots \ldots \ldots \ldots \ldots \ldots \ldots$

4.2 Negative $r$ entries (in percent) of $V_{+} \ldots \ldots \ldots \ldots \ldots \ldots \ldots \ldots \ldots$

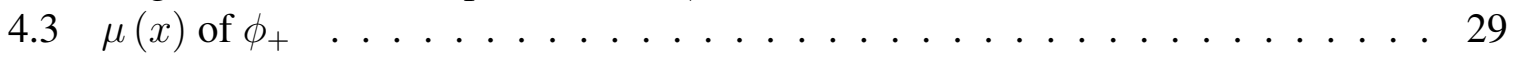

$4.4 \operatorname{var}(x)$ of $\phi_{+} \ldots \ldots \ldots \ldots \ldots \ldots$

4.5 Sweep of $\mathrm{K}$ values to determine normalized $x$ exceeding threshold and classified false-positives. . . . . . . . . . . . . . . . . 35

5.1 Quantifying the change in the spread of $x$ of the Rayleigh distribution during an event. . . . . . . . . . . . . . . . . . . . . . 664 


\section{List of Figures}

1.1 PMU Interface . . . . . . . . . . . . . . . . . . . . . . . . . 2

3.1 Phase and Symmetrical components . . . . . . . . . . . . . . . 13

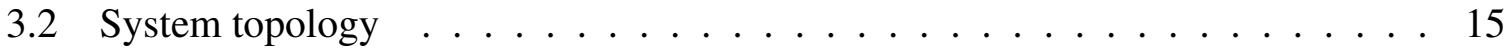

4.1 Time domain plot of $V_{+}$and $\phi_{+}$during event . . . . . . . . . . . . . . 19

4.2 Sample correlation visual . . . . . . . . . . . . . . . . . 21

4.3 Data structure of correlation visual . . . . . . . . . . . . . . 22

4.4 Case study PMU cluster . . . . . . . . . . . . . . . . . . . . . . . . . . 24

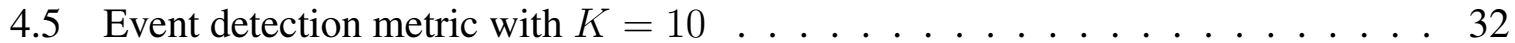

4.6 Event detection metric with $K=100 \quad \ldots \ldots$. . . . . . . . . . . . . 33

4.7 Event detection metric with $K=250 \quad \ldots \ldots \ldots \ldots$. . . . . . . . 34

4.8 Maximum false-positive threshold values for all PMUs . . . . . . . . . . . 36

4.9 Maximum true-negative threshold values for all PMUs . . . . . . . . . . . . 37

4.10 Visual structure characterizing data events . . . . . . . . . . . . . . . 39

4.11 Visual structure during an event . . . . . . . . . . . . . . 41

4.12 Exclude PMUs when MFPTV $>$ MTNTV . . . . . . . . . . . . . . 42

4.13 Cluster on PMUs with low MFPTV . . . . . . . . . . . . . . . . 43

4.14 Time domain plot comparison / impact of $r$ during event for all near cluster . . 45

4.15 Time domain plot comparison / impact of $r$ during event for all far cluster . . . 46

5.1 Time domain plot comparison / impact of $r$ during event for case study cluster . 48

5.2 Impact of $r$ during data drop $\ldots \ldots \ldots \ldots$. . . . . . . . . . . 50

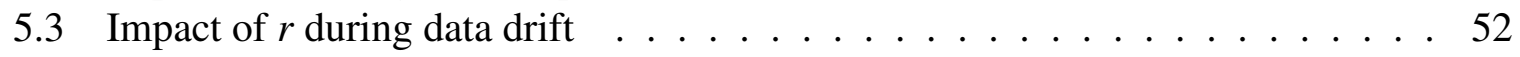

$5.4 \mu(x)$ of $\phi_{+} 13$ cycles into a lightning event $\ldots \ldots \ldots \ldots \ldots$

$5.5 \operatorname{var}(x)$ of $\phi_{+} 13$ cycles into a lightning event $\ldots \ldots \ldots \ldots \ldots$

5.6 Correlation visual of $\phi_{+} 13$ cycles into an event $\ldots \ldots \ldots \ldots$

5.7 Comparison of $\operatorname{var}(x)$ over $V_{+}$and $\phi_{+} \ldots \ldots \ldots \ldots \ldots \ldots$

5.8 Rayleigh distribution of $\phi_{+}$over 15 cycle window size before/during event occurrence . . . . . . . . . . . . . . . . . . 61

5.9 Rayleigh distribution of $\phi_{+}$over 30 cycle window size before/during event occurrence . . . . . . . . . . . . . . . . . . . . 6 62

5.10 Rayleigh distribution of $\phi_{+}$over 60 cycle window size before/during event occurrence . . . . . . . . . . . . . . . . 663

5.11 Event 1: Pre-event normalized $x \ldots \ldots \ldots 6$ 
5.12 Event 1: Normalized $x$ during an event $\ldots \ldots \ldots \ldots$. . . . . . . 68

5.13 Event 1: Normalized $x 40$ cycles into an event $\ldots \ldots \ldots \ldots$

5.14 Event 2: Time domain response 40 cycles into the event . . . . . . . . . 71

5.15 Time domain response over case study cluster for event $2 \ldots \ldots$. . . . . 72

5.16 Visual structure 13 cycles into event $2 \ldots \ldots \ldots$. . . . . . . . 73

5.17 Event 2: Normalized $x$ prior to the event $\ldots \ldots \ldots \ldots \ldots$. . . . . . 74

5.18 Event 2: Normalized $x$. . . . . . . . . . . . . . . . . . . . . . . . 75

5.19 Visual structure 40 cycles into event $2 \ldots \ldots$. . . . . . . . . . 76

5.20 Event 2: Normalized $x 40$ cycles into the event $\ldots \ldots \ldots \ldots$. . . . 77

5.21 Event 3: Time domain response 40 cycles into the event . . . . . . . . . . . 79

5.22 Time domain response over case study cluster for event $3 \ldots \ldots$. . . . . . 80

5.23 Visual structure 13 cycles into event $3 \ldots \ldots \ldots$. . . . . . . . . 81

5.24 Event 3: Normalized $x$ prior to the event $\ldots \ldots \ldots \ldots$. . . . . . . 82

5.25 Event 3: Normalized $x$ during the event $\ldots \ldots \ldots$. . . . . . 83

5.26 Event 3: Normalized $x 40$ cycles into the event $\ldots \ldots \ldots \ldots$

5.27 Visual structure 40 cycles into event $3 \ldots \ldots \ldots$ 


\section{Problem Statement}

Recently, electrical power grids have experienced an influx of renewable generation sources, flexible and dynamic loads, and increased cybersecurity concerns. Pressure from these issues places significant emphasis on accurately determining the current state of the grid. To this end, synchrophasor technology, or Phasor Measurement Units (PMUs), provide a viable solution through real-time grid monitoring. Briefly, PMUs are data collecting devices that capture highly granular, time-stamped phasor measurements of electrical waveforms from electrical power systems. This increased monitoring capability is leading to enhanced levels of observability, grid control, and decision support within grid operation centers. Currently, however, power operations do not yet rely solely on PMU data; real-time applications, such as state estimators and remedial action schema, currently do not utilize the benefits of these high fidelity measurements. The analysis method presented in this thesis offers a statistical-based monitoring scheme purposed for real-time detection of both data events and power flow contingencies with the objective of providing increased awareness to grid operations. 


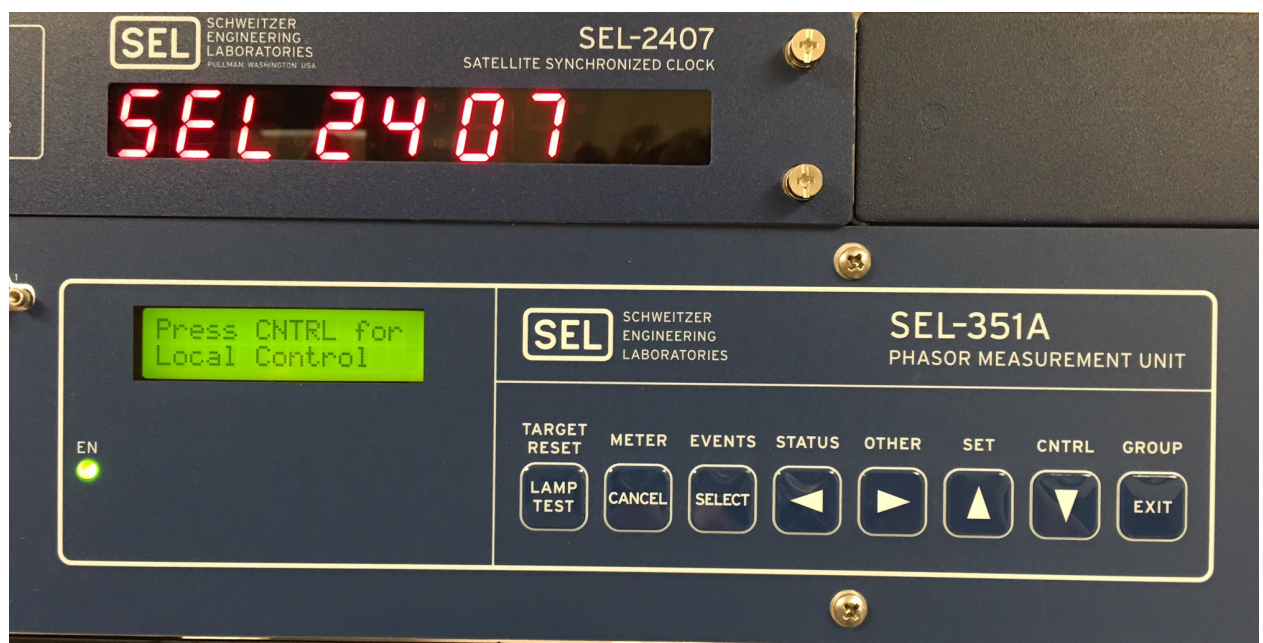

Figure 1.1: Actual Phasor Measurement Unit setup in PGE Power Engineering Lab.

Currently, Supervisory Control and Data Acquisition (SCADA) systems serve as the basis for grid monitoring. SCADA systems sample data every 1 to 4 seconds. Monitoring schema use these data and state estimators to determine power system voltages and power flows. PMU measurements are far superior to SCADA measurements in terms of resolution and accuracy as measurements can be taken at rates up to 120 phasor measurements per second, neglecting the need for state estimation. The high data measurement rate also provides insight into the transient nature of contingencies.

The methods proposed in this thesis use PMU data for detection of both data errors and power system events. The method is a statistically-based middle-ware algorithm intended to inform higher-level applications of abnormal system behavior. For instance, the algorithm could inform remedial action schemes that actuate autonomous control over a power system in response to power system events. Integrity of the data must be ensured in order to avoid insecure operations from occurring. Therefore, the purpose of the presented methods are two-fold: 1) preserve data integrity by timely detection of erroneous data and 2) establishing 
a metric for detecting occurrences of power system events at or near real-time. With this in mind, these methods aim to minimize computation time and allocation of computing resources, necessary characteristics to achieving real-time monitoring and control.

In this thesis, a monitoring stratagem based on a PMU site clustering scheme is introduced. Clustering schemes such as those described by Pierce, et al., have been shown to be highly informative for retrospective detection of previously unidentified events [1]. In our work, PMU clustering is done by selecting a 'sufficient' amount of PMU data streams to monitor in order to optimize monitoring over a particular area within a system while minimizing computational resources and computation time.

By selecting a subset of PMUs from this area, a cluster containing a combination of both electrically-near and electrically-far PMUs, a pairwise comparison between each PMU site's positive sequence phase angle is made using a linear statistical method to create a vector of correlation values. These correlation vectors are then used to define a Rayleigh distribution from which statistical parameters can be quantified. By monitoring clusters of near-far PMU sites and calculating these statistical parameters, metrics for identifying disturbances within this area of the system are established.

The capability of this clustering method, when used in analyzing pairwise positive sequence phase angles to determine statistically significant Rayleigh parameters for event detection, is demonstrated. In addition, its robustness in data and power system event detection is demonstrated with a 'window size' feature (discussed in detail in Chapter 4.2). 


\section{Literature Review}

Over the past few years, extensive research efforts have been made towards event detection using PMU-generated data. These methods can roughly be categorized as statistical algorithms or signal processing algorithms.

In regards to the latter, some efforts are based on Principal Component Analysis (PCA), a mathematical procedure that uses an orthogonal transformation to convert a set of observations of possibly correlated variables into a set of linearly uncorrelated variables called principal components. PCA is a useful statistical technique that is commonly used for finding patterns in data of high dimension. Ge, et al., defined simple rules for voltage and power event detection at the distribution level where voltage events include sags, swells, under/over voltage, and interruptions and power events include load drops and surges [2]. Conversely, rules can be made complex, as described by Xie, et al., in which they provide a dimensionality reduction analysis at the transmission level in order to significantly lower dimensional "signature" of the states in the overall power system to detect line and unit tripping [3].

Additional signal processing-based efforts use an analysis similar to Fourier, called Prony's method, in which a uniform signal is sampled to generate a series of damped complex exponentials. These enable estimation of frequency, amplitude, phase and damping 
components. Sant, et al., use Prony's method to characterize line switching and unit tripping for a handful of events [4].

Yet another type of signal processing technique includes a method for determining the statistical self-affinity of a signal, known as detrended fluctuation analysis (DFA), as discussed by Ashton, et al., in which analysis over system frequency is performed to determine values of fluctuation [5]. These values can then determine line and unit tripping occurrences.

The most relevant effort that closely resembles the methods proposed in this thesis was developed by Sohn, et al., [6]. This work uses both statistical and signal processing techniques, namely, linear analysis, residual modeling, and short-time Fourier transforms. Based on these methods, parameters, such as mean, variance, and correlation, were determined. For each of these parameters, which they refer to as indices, a set of decision rules are defined in order to provide indication of an event occurrence.

This work closely resembles the methods covered in this thesis in a few ways. They are conceptually similar in that statistical parameters are determined from statistical methods and are used to derive metrics for event detection. Additionally, the defined rules provide indication of an event occurrence in a similar fashion; an event will be declared if these parameters exceed some established threshold value. Another similarity is the challenge of determining an appropriate threshold value.

This work is also different than the methods proposed in this thesis in a few ways. First, the methods covered in this thesis are solely statistical-based. Second, the statistical methods 
are different; while this work does not explicitly state the statistical methods, it is assumed that they are not the same. Lastly, this work relies on a majority of the indices to give indication of the event. This thesis uses a single detection metric but indication of an event is provided by clustered PMUs.

In general, the most challenging issue with a statistical-based approach is determining a threshold value over statistical parameters. This has been a known issue for decades as discussed with earlier research found in [7]; "If the phenomenon is ill-understood or changes its behavior unpredictably, adapting the threshold such that event reporting is accurate becomes very difficult." This thesis work provides a methodical approach to addressing this challenge when establishing an appropriate threshold value. 


\subsection{Intermediate Work}

During the initial phase of this research a collaborative effort was made between Oregon State University (OSU), Washington State University Vancouver (WSUV), and Portland State University (PSU) to develop a middle-ware algorithm which would analyze PMU data and inform higher-level applications of power system event occurrences. Because higherlevel applications were to act on this information, data integrity needed to be ensured in order to avoid insecure operations being performed. This algorithm, entitled the Correlation Matrix Algorithm (CMA), streams all PMU data into a standard input (stdin) process in which an algorithm reads from to perform pairwise correlation between all PMUs. This correlation uses a linear statistical method known as Pearson correlation and is represented quantitatively in a visual structure which is updated on a per-single cycle basis for all PMU pairs. This work was published in the IEEE Technologies for Sustainability (SusTech) in 2014 [8] and will be discussed in further detail in this thesis.

In conjunction with this work, a data management methodology was developed to contend with the big data issues that arise due to the high fidelity rate of PMUs. This method, known bitmap indexing, scans raw PMU data values and 'bins' them so that they can be stored as binary vectors. In this fashion, when data comparisons need to be made, Boolean algebra is used to recall data specified by some criteria, greatly reducing retrieval times. Using Boolean algebra to retrieve data is shown to be much quicker and more effective than performing analytical comparisons. This work was also published in the IEEE Technologies for Sustainability (SusTech) in 2014 [9]. 
In addition to event detection, this work takes a cybersecurity perspective and investigates detection of spoofing attacks. These attacks, most notably used with the stuxnet virus [10], are becoming increasingly common. These attacks are typically prerequisite to nefarious activity; signals will be spoofed as legitimate in order to mask malicious intent. Using both correlation methods and support vector machine classifiers, a variety of spoofing attacks were created and injected into nominal data streams in order to evaluate the detection performance from these individual methods. Both methods show to be highly viable in detecting these types of attacks. This work was published in the IEEE Transactions in Smart Grid [11]. 


\section{Background}

This section provides background information on the various aspects related to the project. These include the synchrophasor IEEE standard to provide a brief insight into the capabilities of synchrophasor technology, a power system method known as sequence components to provide context on the data included in the dataset, details on the characteristics of the PMUgenerated dataset captured from a real-world power system, and a simplified topological representation of the synchrophasor network contained in the dataset.

\subsection{Synchrophasor IEEE Standard}

The concept of a synchronized power system phasor was first standardized with the standard IEEE 1344 [12]. This standard has since been replaced by the latest standard, IEEE C37.1182011 [13]. Among others, this standard explicitly defines phasor measurements so that measurement equipment can be readily interfaced with associated systems. The standard provides a method of quantifying measurements, and it defines quality test specifications. The latest version of the standard accomplishes this for the power system frequency, $f$, and its derivative, the rate of change of frequency (ROCOF). 


\subsubsection{Phasor Definition}

A phasor is a mathematical representation of a sinusoidal function that exists in the complex plane. Sinusoids of the form $x(t)=X_{m} \cos (\omega t+\phi)$, where $\phi$ is its instantaneous phase angle, are commonly represented in phasor representation as,

$$
\begin{aligned}
\mathbf{X} & =X_{r}+j X_{i}=\left(X_{m} / \sqrt{2}\right) e^{j \phi} \\
& =X e^{j \phi}
\end{aligned}
$$

where $X=X_{m} / \sqrt{2}$ is the root mean square (RMS) value of the signal $x(t)$. This representation of sinusoidal signals measured from power systems is adopted in this standard.

Under this definition, $\phi$ is the offset from a cosine function at the nominal system frequency synchronized to Universal Time Coordinated (UTC). Therefore, in order to determine $\phi$, UTC must be provided. Due to the precise timing requirements, as described in the following section, $\phi$ is determined with a high degree of accuracy.

\subsubsection{Timetags and Synchronization}

Phasor measurements are tagged with UTC time corresponding to the time of measurement. Timetags consist of a second-of-century (SOC) count, a fraction-of-second count, and a time of status value. UTC is the primary time standard by which the world regulates clocks and time. Phasor measurements coupled with UTC time stamps are referred to as 'synchrophasors,' a term that will be used henceforth. 
To provide sufficient time accuracy, systems must be capable of receiving time from a highly reliable source such as Global Position System (GPS). Measurements must be synchronized to UTC time with adequate sufficiency in order to meet accuracy requirements (described next in Chapter 3.1.3). For reference, a time error of $1 \mu$ s corresponds to a phase error of $0.022^{\circ}$ for a $60 \mathrm{~Hz}$ system. A phase error of $0.57^{\circ}$ will cause a $1 \%$ total vector error (TVE - detailed below). This corresponds to a maximum time error of $\pm 26 \mu \mathrm{s}$.

\subsubsection{Measurement Requirements}

PMUs must support data reporting at submultiples of nominal power system frequency but up to system frequency is preferred, 60 phasor measurements per second. Response times must stay in the specified accuracy zone corresponding to a $1 \%$ compliance level in the TVE.

Under the conditions where $X_{m}, \omega$, and $\phi$ are fixed, the total vector error (TVE) is defined to be an expression of the difference between a "perfect" sample of a theoretical synchrophasor and the estimate given by the unit under test at the same instant of time. The value is normalized and expressed as per unit of the theoretical phasor and is defined, as seen in the standard [13], by the following equation:

$$
T V E(n)=\sqrt{\frac{\left(\hat{X}_{r}(n)-X_{r}(n)\right)^{2}+\left(\hat{X}_{i}(n)-X_{i}(n)\right)^{2}}{\left(X_{r}(n)^{2}+X_{i}(n)^{2}\right)}}
$$

where $\hat{X}_{r}$ and $\hat{X}_{i}$ are measured values and $X_{r}$ and $X_{i}$ are theoretical values. Although this research does not directly deal with TVE, it is included here to demonstrate the accuracy and precision of the measurements taken. 


\subsection{Sequence Components}

To better comprehend the characteristics of the dataset used in this research, providing context on a method known as sequence components, is first warranted. The setting of this research is at the transmission level, with nominal voltages set to $500 \mathrm{kV}$. Transmission systems are commonly 3-phase. Each line, known as a phase component, e.g. $I_{A}, I_{B}$, and $I_{C}$ representing the line current phasors, are $120^{\circ}$ out of phase, with respect to each other. Systems that operate in this fashion are said to be balanced.

During fault conditions, such as a line-to-ground fault, the system becomes unbalanced. Analyzing phase components during these conditions is often difficult. To facilitate ease in analyzing the system during these conditions, a technique can be performed in which a set of unbalanced phase components can be transformed to sets of balanced symmetrical components.

Symmetrical components, denoted $I_{0}, I_{1}$, and $I_{2}$, constitute a set of balanced current phasors representing zero, positive, and negative sequence. This sequence indicates the rotational direction, in the phase plane, in which these phasors cross a reference point, typically at the $0^{\circ}$ point. Each phase component can be represented as a combination of zero, positive, and negative sequence phasors. Figure 3.1 shows the matrix representation of both phase and symmetrical components (left and right, respectively) of the line current phasors, with $\alpha$ representing a $+120^{\circ}$ phase shift. 


$$
\left[\begin{array}{c}
\mathrm{I}_{\mathrm{A}} \\
\mathrm{I}_{\mathrm{B}} \\
\mathrm{I}_{\mathrm{C}}
\end{array}\right]=\left[\begin{array}{ccc}
1 & 1 & 1 \\
1 & \alpha^{2} & \alpha \\
1 & \alpha & \alpha^{2}
\end{array}\right]\left[\begin{array}{l}
\mathrm{I}_{0} \\
\mathrm{I}_{1} \\
\mathrm{I}_{2}
\end{array}\right] \quad\left[\begin{array}{l}
\mathrm{I}_{0} \\
\mathrm{I}_{1} \\
\mathrm{I}_{2}
\end{array}\right]=\frac{1}{3}\left[\begin{array}{ccc}
1 & 1 & 1 \\
1 & \alpha & \alpha^{2} \\
1 & \alpha^{2} & \alpha
\end{array}\right]\left[\begin{array}{l}
\mathrm{I}_{\mathrm{A}} \\
\mathrm{I}_{\mathrm{B}} \\
\mathrm{I}_{\mathrm{C}}
\end{array}\right]
$$

Figure 3.1: (Left) Phase components and (Right) Symmetrical components of the line currents in phasor form. If a set of components is known, the other can be determined from a matrix transformation. $\alpha$ representing a $+120^{\circ}$ phase shift.

In general, positive sequence voltage data is most often considered in analysis of grid operations mainly because these values reflect the stability of active power flow on the grid. This is especially true when workable, though often approximate, power flow solutions are desired during nominal, balanced, 3-phase operation. This research involves analysis of positive sequence voltage data. 


\subsection{Dataset Characteristics}

This research uses synchrophasor datasets provided by the Bonneville Power Administration (BPA). These contain archived, operational data captured from within BPA's balancing area. These data conform to IEEE C37.118-2011 (described above in section 3.1) and consists of a single year's worth of data spanning August 2012 to August 2013 from twenty PMU sites. The data set size is $950 \mathrm{~GB}$. Each file in the set typically holds one to five minutes of data from each of the twenty separate PMU sites. It includes both positive sequence voltage magnitude $(\mathrm{V}+)$ and positive sequence voltage phase angle $\left(\phi_{+}\right)$.

For this work, analysis is centered around $\phi_{+}$. During nominal operations, $\phi_{+}$varies slowly in contrast to $V+$, which can experience sudden changes. Furthermore, $\phi_{+}$trends similarly between adjacent PMU sites whereas $V+$ can be at different levels along adjacent PMU sites and is also allowed to deviate $\pm 10 \%$ from its nominal voltage of $500 \mathrm{kV}$ (1 per unit [p.u.]). Because of these reasons, the methods presented are shown to be highly sensitive to sudden changes in $\phi_{+}$, making it the desirable parameter for use in the analysis. Justification of this will be reiterated in Chapter 5.3.

Each measurement is given in synchrophasor representation with its associated date/time stamp. The discretization rate between measurements is 16.7 milliseconds (60 phasor measurements/second). The phase angle $\phi_{+}$is a time-varying real number that oscillates within the range of $\pm 180^{\circ}$, whereas the voltage magnitude is a non-negative real number. 


\subsection{Network Topology}

In regards to the synchrophasor network topology, Figure 3.2 provides a general layout of PMU site locations. Although not all PMU sites shown are contained in the dataset (dotted busses are not included) they are incorporated for the sake of completeness.

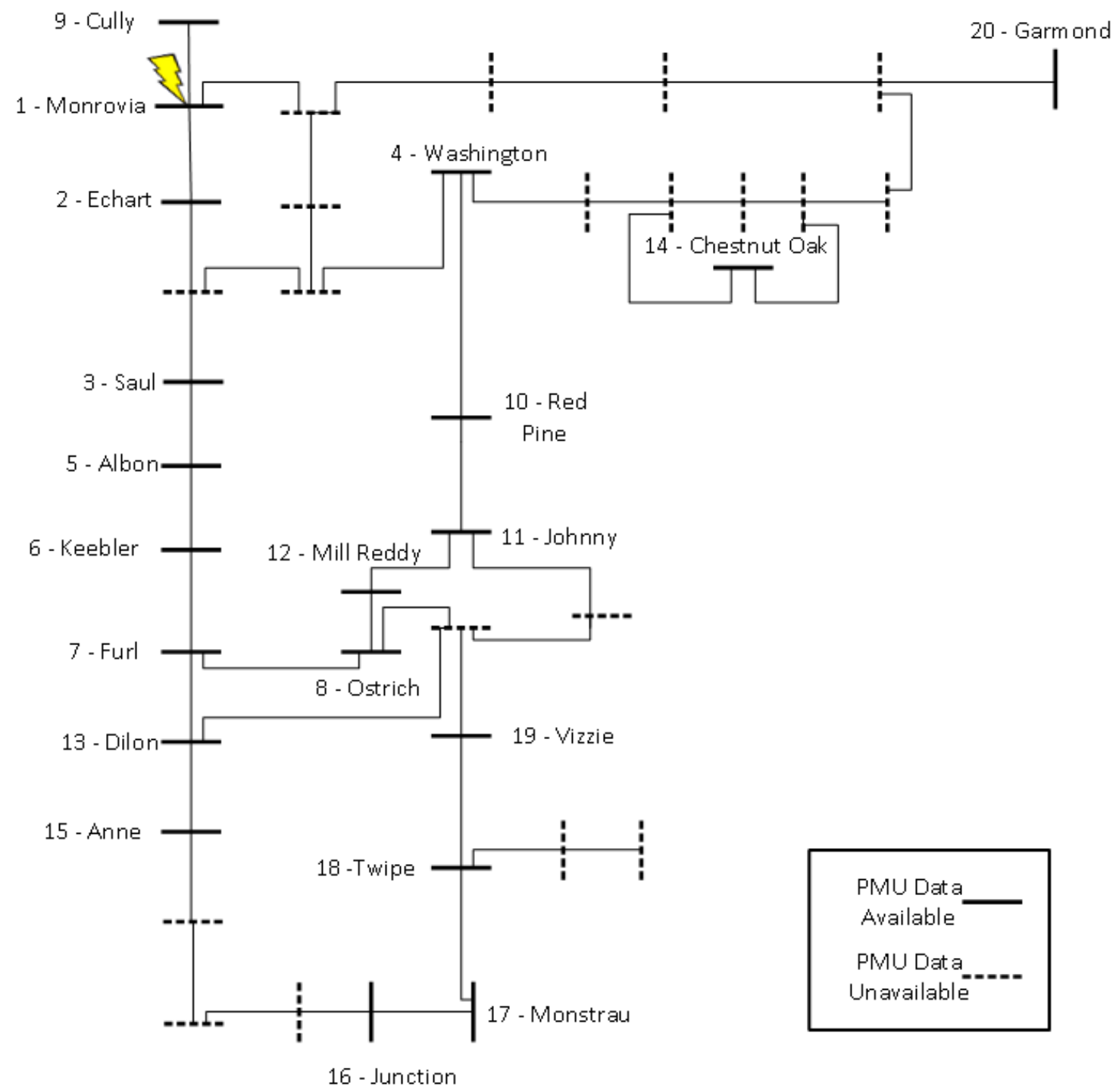

Figure 3.2: Synchrophasor network topology represented as a one-line diagram. PMU sites found in the dataset are indicated by solid busses. A lightning strike occurs near PMU site Monrovia and will be presented as a case study. Numbers near site names represent the next closest site to the lightning strike in terms of electrical distance (discussed in further detail in Chapter 4.4). 
Our algorithm is tested against power system events that occurred within this system during the August 2012 to August 2013 time period. Instances of lightning strike occurrences have been recorded in the northwest region of Figure 3.2 near PMU site Monrovia. Each instance led to an occurrence of a line-to-ground fault. Numbers near site names represent the next closest site to the fault in terms of electrical distance (a concept covered in Chapter 4.4). These are the power system events that serve as test cases presented in Chapter 5. 


\section{Design Methodology}

\subsection{Correlation}

Correlation is a common statistical method used to determine if relationships exist between two continuous variables. The method presented here utilizes a linear statistical method known as Pearson correlation. To assess the linear relationship between variables, the Pearson correlation coefficient (PCC), $r$, from recent works [14], is determined based on the following equation:

$$
\begin{aligned}
r & =\frac{\operatorname{cov}(X, Y)}{\sigma_{X} \sigma_{Y}} \\
& =\frac{\Sigma(X Y)-\frac{\Sigma X \Sigma Y}{N}}{\sqrt{\left(\Sigma\left(X^{2}\right)-\frac{(\Sigma X)^{2}}{N}\right) \times\left(\Sigma\left(Y^{2}\right)-\frac{(\Sigma Y)^{2}}{N}\right)}}
\end{aligned}
$$

where cov is the covariance, $\sigma_{X}$ is the standard deviation of $\mathrm{X}, \sigma_{Y}$ is the standard deviation of $\mathrm{Y}$, and $\mathrm{X}$ and $\mathrm{Y}$ are two independent continuous variables of size $\mathrm{N}$.

The output of $r$ is such that $-1 \leq r \leq 1$ where $r=-1$ represents a perfectly negative linear relationship, $r=1$ represents a perfectly positive linear relationship, and $r=0$ represents an inconclusive relationship. It has been established that $|r| \geq 0.9$ can be interpreted as being 'very highly' correlated, $0.7 \geq|r|<0.9$ as 'highly' correlated, $0.5 \geq|r|<0.7$ as 'moderately' correlated, and $0.3 \geq|r|<0.5$ as having 'low' correlation. 


\subsection{Window Length}

From Equation 4.1, the independent continuous variables, $\mathrm{X}$ and $\mathrm{Y}$, represent vectors of data from particularly selected PMU sites. These vectors can be either positive sequence voltage magnitude, $V_{+}$, or phase angle $\phi_{+}$. Since $\mathrm{X}$ and $\mathrm{Y}$ are of size $\mathrm{N}$, altering the size over a variety of range values can serve as a feature enabling increased robustness and speed in detection of both data and power system events due to the fact that the cardinality of $\mathrm{N}$ represents the 'window size' of correlation when determining $r$.

Due to the transient nature of power system events, the window size must be selected to be small enough to detect an event in a timely manner and large enough to capture the dynamics of the event. In Chapter 5 we show that varying the window size increases robustness at detecting different types of data events and in detecting actual power system events that occur within real systems, but at the cost of increased computational intensity.

In addition, entries in both $\mathrm{X}$ and $\mathrm{Y}$ are updated on a cycle-by-cycle basis permitting $\mathrm{N}$ to serve as a 'sliding window' over $V_{+}$and $\phi_{+}$data. Figure 4.1 shows both $V_{+}$and $\phi_{+}$data during an event and demonstrates both sliding window and window size concepts. 

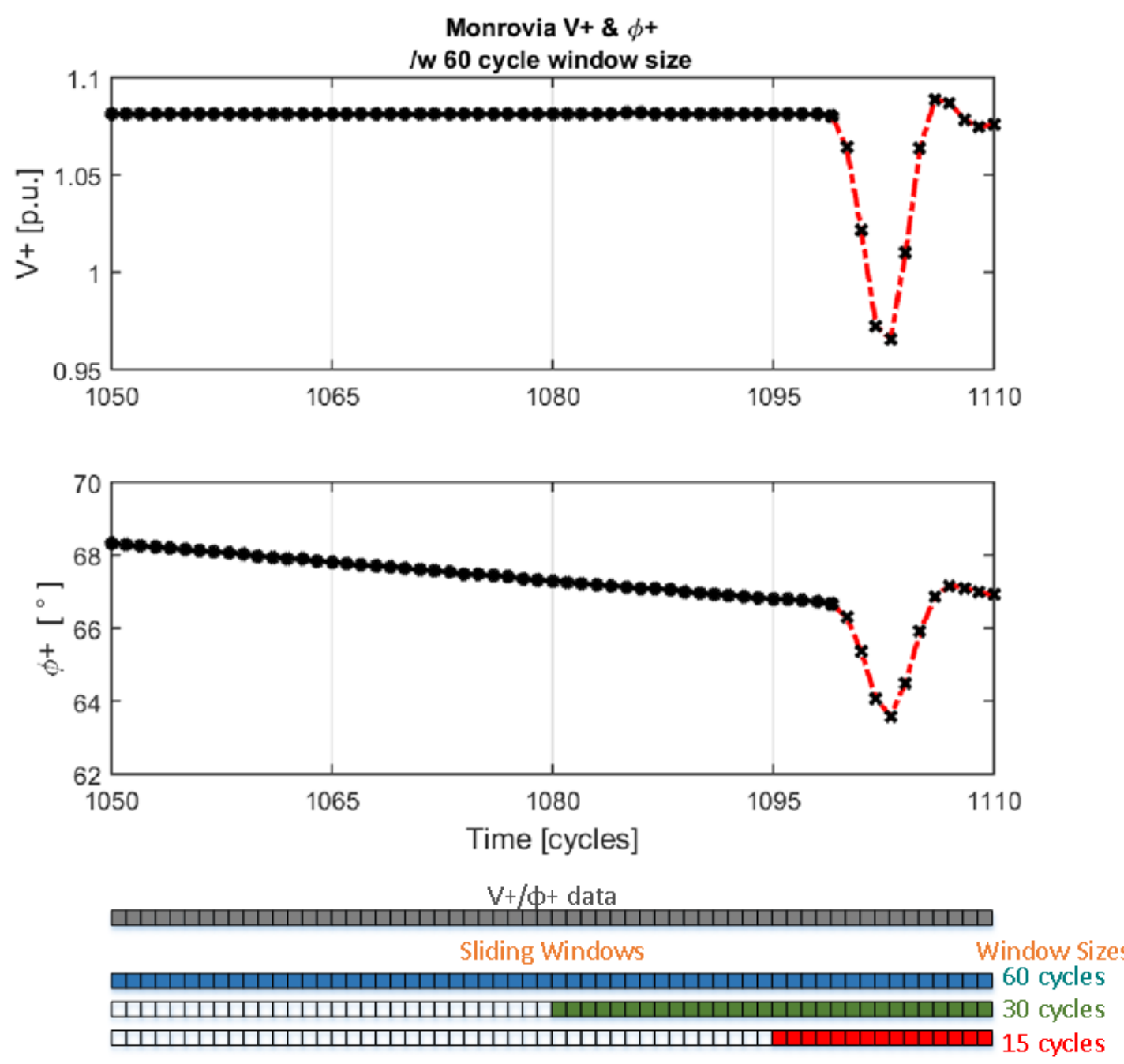

Figure 4.1: Time domain plot of $V_{+}$and $\phi_{+}$data over a 60 cycle window size during a case study event. Sliding windows with associated window size are depicted at the bottom of the figure. Sliding windows of size $\mathrm{N}$ determine the value of $r$. 


\subsection{Correlation Visual}

During the early phases of this research, a collaborative effort was made between Oregon State University (OSU), Washington State University Vancouver (WSUV), and Portland State University (PSU) to develop an event detection algorithm dubbed Correlation Matrix Algorithm (CMA). The CMA facilitates the use of real-time PMU data for detecting and flagging data and event-related issues. Another result of this collaboration was the creation of an engine that simulates real-time streaming of PMU data. This data stream serves as input into our correlation methods, and provides a visual representation of system-wide correlation, as shown in Figure 4.2.

To explain Figure 4.2, let us be explicit of its features. Each coordinate square represents a pairwise correlation value (indicated by the color scale on the right) between 'FROM' and 'TO' PMU numbers. The structure takes an upper triangular shape due to the pairwise nature of correlation. The sign of the correlation is indicated in the center of each coordinate square as either $\mathrm{a}+$, or - symbol. When correlation is inconclusive, a $\emptyset$ symbol will be present and the coordinate square will be blacked out. The title, at the top of the figure, indicates the attribute $\left(V_{+}\right.$or $\left.\phi_{+}\right)$, window size, and time of latest entry (in seconds) being analyzed. The sections below provide a brief overview of this work. 


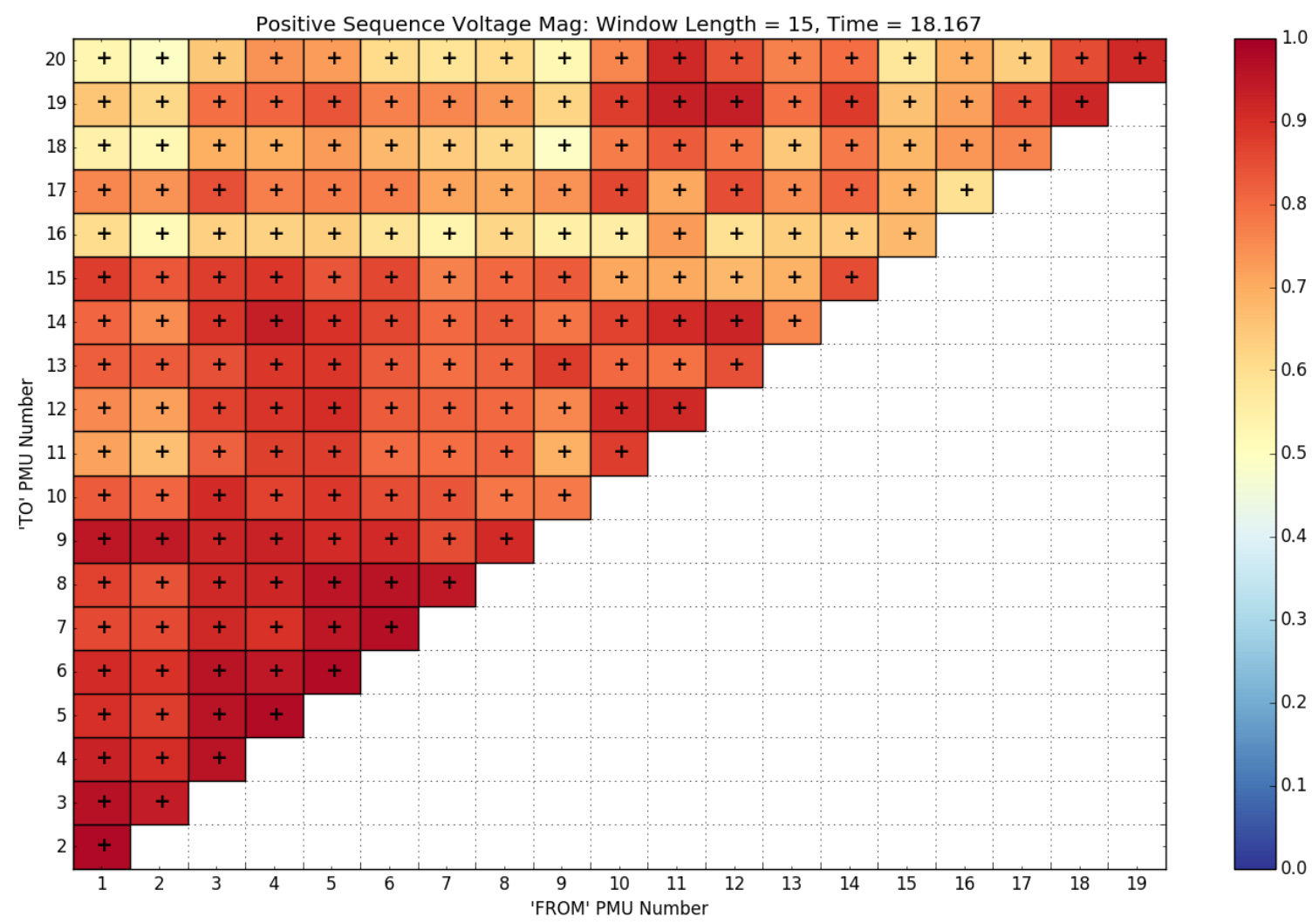

Figure 4.2: Visual representation of system-wide correlation utilizing a 15 cycle window size over nominal $V_{+}$ data (void of any data/event-related issues) for a system containing 20 PMUs.

\subsubsection{Historic Playback Engine}

This collaboration resulted in co-development of a real-time, data-playback engine that analyzes characteristics of the dataset. The engine streams archived PMU data as input into the CMA effectively linking raw power system data and our correlation methodology.

By understanding the file traits, recorded power system attributes, data discretization rate, and topological layout of the PMUs (depicted in Figure 3.2), a Phasor Data Concentrator $(P D C)$ engine was created. Often, PMUs located at different points around the grid are grouped by zones, and consequently their data streams are multiplexed to a single data 
logging point. This central data entry point is what is commonly referred to as a PDC. The PDC engine serves a similar purpose by replicating a single multiplexed data stream based on the archived operational PMU data, thus simulating the real-time power system operation.

\subsubsection{Data Structure}

As positive sequence voltage data is processed in the time-domain by the PDC engine, the data are written into the working memory of the correlation algorithm. In an effort to minimize computational complexity, a custom data structure was developed to quickly append new data, reference data already stored, and account for multiple characteristics such as time, magnitude, phase, and correlation coefficients for each of the 20 PMUs. This versatile data structure is depicted in Figure 4.3.

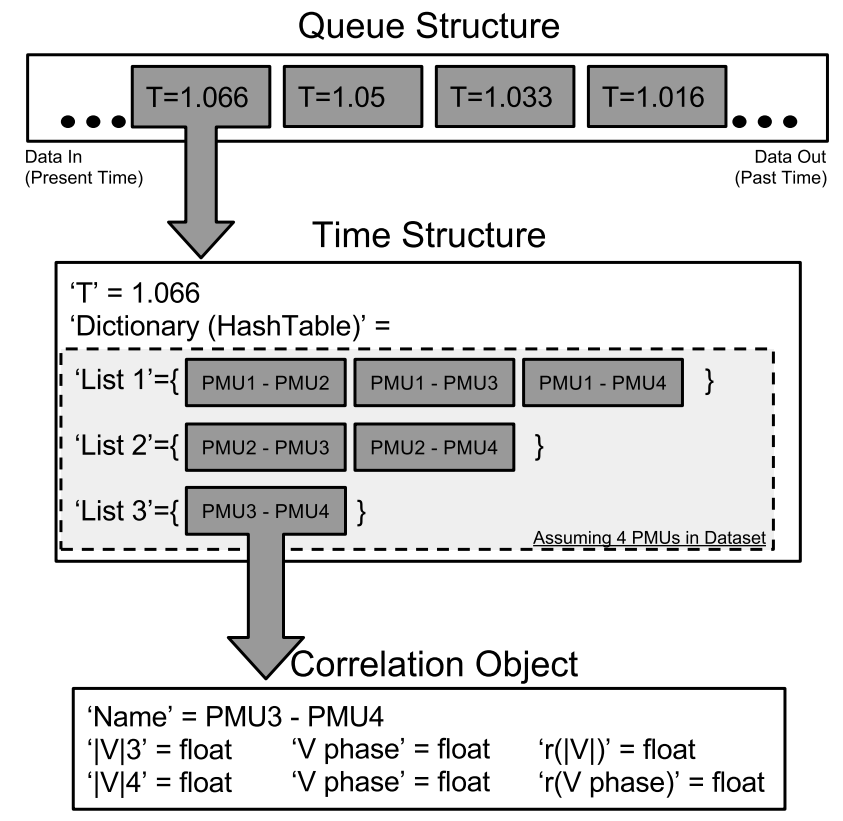

Figure 4.3: The 3-tiered data structure used to store PMU data for the PDC engine. (Figure created by Rich Meier at OSU) 
As seen in Figure 4.3, the lowest layer of the data management system holds the actual values read in from the PDC feeder as well as the calculated correlation coefficients (referred to as "Correlation Objects"). These Correlation Objects are dynamically created based on the number of multiplexed PMUs. This reduces redundancy of data in the correlation technique since Correlation Objects represent a combination of two PMUs. The next layer of the management system is made up of Correlation Object combinations at a specific time stamp (referred to as "Time Structure"). This creates a triangular matrix of Correlation Objects that reference both a unique time as well as the PMU data and correlation value at that particular time. Finally, at the highest management level, each Time Structure is stored in a queue of dynamic length. The PMU and correlation data can effectively be monitored in the time domain for any desired window size. 


\subsection{Electrical Distance}

The concept of electrical distance is applied when deciding which PMUs to cluster. Electrical distance give a sense for the electrical proximity between substations, as opposed to physical distance. As such, two electrically close substations will experience similar responses to a nearby system event, whereas two substations that are electrically-far from each other may not exhibit similar responses to an event. The concept of electrical distance has been applied to multiple different scenarios, such as multi-objective power network partitioning, identifying structural vulnerabilities, and evaluating marginal loss factors [15-17].

Recall earlier that the Northwest region of Figure 3.2 experienced lightning strike occurrences near PMU site Monrovia. Figure 4.4 focuses on this region. All available PMUs in this region are labeled in ascending order in terms of electrical distance with respect to site Monrovia and will represent a PMU cluster for a particular case study in Chapter 5.

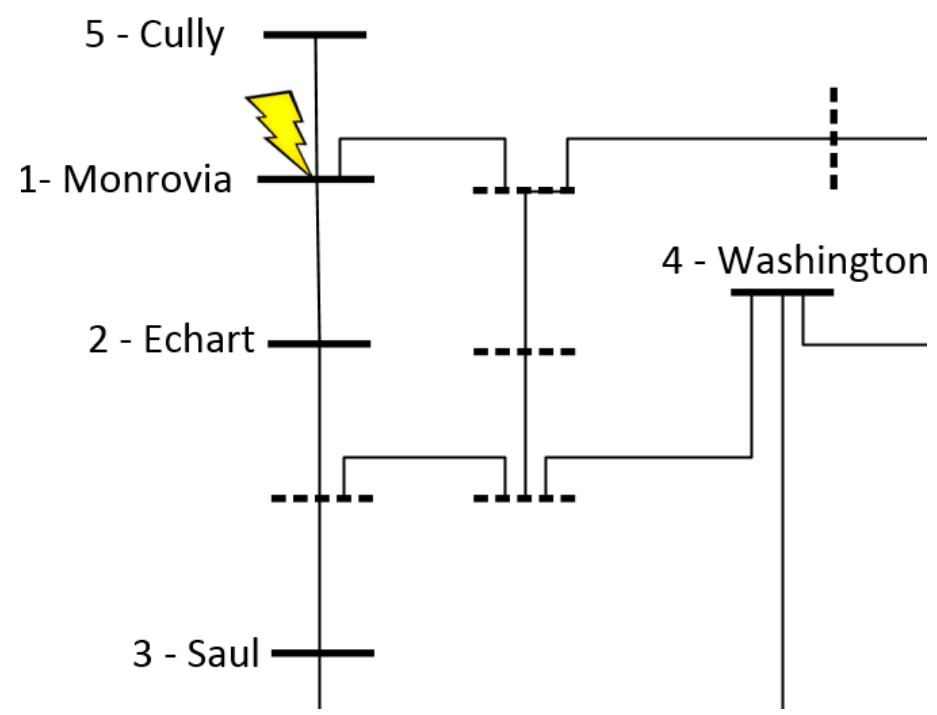

Figure 4.4: One-line of subset PMUs. Numbers indicate next electrically closest site to event (ascending). This particular PMU cluster serves as a case study in Chapter 5. 


\subsection{Rayleigh Distribution}

The distribution to be utilized is a continuous probability distribution known as the Rayleigh distribution, given by recent works [18], is defined as

$$
f(x ; \sigma)=\frac{x}{\sigma^{2}} e^{\left(-\frac{x^{2}}{2 \sigma^{2}}\right)} \mid x \geq 0, \sigma>0
$$

where $x$ is the Rayleigh distribution parameter and $\sigma$ is the scale parameter with

$$
\sigma=\sqrt{\frac{1}{2 n} \sum_{i=1}^{n} x_{i}^{2}}
$$

Applications of this distribution can be found in a variety of areas including communications, image processing, and physical sciences, among others [19-21]. Considering the probability distribution function, selection of this particular distribution was based on the range of values the random variable will be expected to take given it will consist mostly of 'very highly' (positive) correlated $r$ values.

However, the range of the Pearson correlation coefficient, $r$, is such that $r \in[-1,1]$. Noting that very few of the PMU $r$ values are negatively correlated, fewer than $1 \%$ for $\phi_{+}$ (10\% for $V_{+}$), we consider just the magnitude of $r$, for which $|r| \in[0,1]$, or, rather more namely, its inverse,

$$
y=\frac{1}{|r|}
$$

for which $y \in[1, \infty)$. By defining $y$ such that $y=x+1$, the Rayleigh distribution variable can be formally defined as, 


$$
x=y-1=\frac{1}{|r|}-1
$$

for which $x \in[0, \infty)$.

To follow up on the assumption that solely the magnitude of $r$ can be considered, Table 4.1 shows, for the case study PMU cluster, the amount of negative $r$ entries (in percentage) of $\phi_{+}$over a single minute, 1, 2, and 24 hours of nominal data (void of any data/event-related issues) with 15, 30, and 60 cycle window sizes. It can be observed that there are very few occurrences of negative $r$ values of $\phi_{+}$, less than $0.33 \%$ at worst (Washington, 30 cycles, 1 minute - seen in bold). Since the amount of negative $r$ entries of $\phi_{+}$is disproportionate, these entries can be discarded when analyzing $|r|$ of $\phi_{+}$.

Considering $V_{+}$, Table 4.2 shows the amount of negative $r$ entries when applying the same treatment. It can be observed that up to $6.44 \%$ (Washington, 15 cycles, 24 hours - seen in bold) of $r$ entries for $V_{+}$are negatively correlated. Therefore, the assumption that $|r|$ can solely be considered needs to be revisited for analysis of $V_{+}$. This assumption is reiterated and justified for $\phi_{+}$in Chapter 5.3. 
Table 4.1: Negative $r$ entries (in percent) of $\phi_{+}$for case study PMU cluster over a single minute, 1, 2, and 24 hours of nominal data with 15,30 , and 60 cycle window sizes.

\begin{tabular}{l|cccc}
\hline 15 cycles & Echart & Saul & Washington & Cully \\
\hline 1 minute & 0.00 & 0.08 & 0.14 & 0.08 \\
1 hour & 0.02 & 0.16 & 0.19 & 0.17 \\
12 hours & 0.01 & 0.15 & 0.17 & 0.12 \\
24 hours & 0.01 & 0.16 & 0.17 & 0.1 \\
\cline { 1 - 1 } 30 cycles & & & & \\
\cline { 1 - 1 } 1 minute & 0.00 & 0.00 & $\mathbf{0 . 3 3}$ & 0.17 \\
1 hour & 0.00 & 0.08 & 0.15 & 0.12 \\
12 hours & 0.01 & 0.10 & 0.13 & 0.10 \\
24 hours & 0.01 & 0.10 & 0.14 & 0.08 \\
\cline { 1 - 1 } 60 cycles & & & & \\
\cline { 1 - 1 } 1 minute & 0.00 & 0.00 & 0.00 & 0.00 \\
1 hour & 0.00 & 0.04 & 0.07 & 0.05 \\
12 hours & 0.00 & 0.03 & 0.06 & 0.04 \\
24 hours & 0.00 & 0.04 & 0.06 & 0.03
\end{tabular}

Table 4.2: Negative $r$ entries (in percent) of $V_{+}$for case study PMU cluster over a single minute, 1, 2, and 24 hours of nominal data with 15,30 , and 60 cycle window sizes.

\begin{tabular}{l|cccc}
\hline 15 cycles & Echart & Saul & Washington & Cully \\
\hline 1 minute & 0.08 & 1.00 & 1.03 & 1.75 \\
1 hour & 0.51 & 4.11 & 4.47 & 2.72 \\
12 hours & 0.99 & 5.81 & 6.03 & 2.60 \\
24 hours & 1.14 & 5.95 & $\mathbf{6 . 4 4}$ & 2.55 \\
\cline { 1 - 1 } 30 cycles & & & & \\
\cline { 1 - 1 } 1 minute & 0.00 & 0.03 & 0.72 & 0.89 \\
1 hour & 0.04 & 2.46 & 3.31 & 1.55 \\
12 hours & 0.11 & 3.54 & 4.10 & 1.37 \\
24 hours & 0.13 & 3.36 & 4.05 & 1.17 \\
\cline { 1 - 1 } 60 cycles & & & & \\
\cline { 1 - 1 } 1 minute & 0.00 & 0.00 & 0.06 & 0.14 \\
1 hour & 0.00 & 1.22 & 3.16 & 0.92 \\
12 hours & 0.01 & 2.02 & 3.43 & 0.86 \\
24 hours & 0.01 & 1.76 & 2.93 & 0.67
\end{tabular}

Refocusing on characteristics of the Rayleigh distribution, in addition to the Rayleigh random variable $x$, two pertinent statistical parameters of this distribution are the mean and 
variance, from recent works [18], are defined as,

$$
\mu(x)=\sigma \sqrt{\frac{\pi}{2}}
$$

and

$$
\operatorname{var}(x)=\frac{4-\pi}{2} \sigma^{2}
$$

For electrically close PMUs in steady-state, the expected value of $|r|$ is very close to one, since under steady-state conditions the voltage magnitude and phase angle profiles trend nearly identically. Therefore, deviations from $|r|=1$, are very rare. As such, the expected value for $x$ is near zero. This is, in fact, what we observe; observing $\mu(x)$ and $\operatorname{var}(x)$ for PMU pairs included in the case study, it can be seen that there is little variation from steady-state values. Table 4.3 and 4.4 show, for the case study PMU cluster, calculated $\mu(x)$ and $\operatorname{var}(x)$ over a single minute, 1, 2, and 24 hours of nominal data with 15, 30, and 60 cycle window sizes. $V_{+}$is not given similar treatment and justification for doing so will be provided in Chapter 5.3. 
Table 4.3: $\mu(x)$ of $\phi_{+}$for case study PMU cluster over 15, 30, and 60 cycle window size.

\begin{tabular}{|c|c|c|c|c|}
\hline 15 cycles & Echart & Saul & Washington & Cully \\
\hline 1 minute & $1.20 \mathrm{E}-02$ & $9.40 \mathrm{E}-03$ & $1.30 \mathrm{E}-02$ & $6.70 \mathrm{E}-03$ \\
\hline 1 hour & 4.00E-03 & $5.00 \mathrm{E}-03$ & $6.20 \mathrm{E}-03$ & 4.70E-03 \\
\hline 12 hours & $3.40 \mathrm{E}-03$ & 4.80E-03 & $6.00 \mathrm{E}-03$ & 4.40E-03 \\
\hline 24 hours & $3.10 \mathrm{E}-03$ & $4.30 \mathrm{E}-03$ & $5.60 \mathrm{E}-03$ & 4.10E-03 \\
\hline 30 cycles & & & & \\
\hline 1 minute & $1.3 \mathrm{E}-02$ & $1.2 \mathrm{E}-02$ & 7.0E-03 & 7.3E-03 \\
\hline 1 hour & $2.6 \mathrm{E}-03$ & 4.9E-03 & $6.1 \mathrm{E}-03$ & 4.1E-03 \\
\hline 12 hours & $2.1 \mathrm{E}-03$ & 4.2E-03 & $5.7 \mathrm{E}-03$ & $3.8 \mathrm{E}-03$ \\
\hline 24 hours & $2.0 \mathrm{E}-03$ & $4.0 \mathrm{E}-03$ & $5.4 \mathrm{E}-03$ & $3.5 \mathrm{E}-03$ \\
\hline 60 cycles & & & & \\
\hline 1 minute & $6.1 \mathrm{E}-03$ & 8.3E-03 & $9.0 \mathrm{E}-03$ & $1.3 \mathrm{E}-02$ \\
\hline 1 hour & $1.2 \mathrm{E}-03$ & $3.1 \mathrm{E}-03$ & $5.7 \mathrm{E}-03$ & $3.9 \mathrm{E}-03$ \\
\hline 12 hours & $1.1 \mathrm{E}-03$ & $3.4 \mathrm{E}-03$ & $6.2 \mathrm{E}-03$ & $3.0 \mathrm{E}-03$ \\
\hline 24 hours & $1.1 \mathrm{E}-03$ & $3.2 \mathrm{E}-03$ & $6.1 \mathrm{E}-03$ & $2.9 \mathrm{E}-03$ \\
\hline
\end{tabular}

Table 4.4: $\operatorname{var}(x)$ of $\phi_{+}$for case study PMU cluster over 15, 30, and 60 cycle window size.

\begin{tabular}{|c|c|c|c|c|}
\hline 15 cycles & Echart & Saul & Washington & Cully \\
\hline 1 minute & $1.50 \mathrm{E}-03$ & $2.70 \mathrm{E}-03$ & $2.30 \mathrm{E}-03$ & $1.90 \mathrm{E}-03$ \\
\hline 1 hour & $5.90 \mathrm{E}-04$ & $1.30 \mathrm{E}-03$ & 2.30E-03 & $1.30 \mathrm{E}-03$ \\
\hline 12 hours & $5.60 \mathrm{E}-04$ & $1.40 \mathrm{E}-03$ & $2.10 \mathrm{E}-03$ & $1.20 \mathrm{E}-03$ \\
\hline 24 hours & $5.40 \mathrm{E}-04$ & $1.40 \mathrm{E}-03$ & $2.10 \mathrm{I}$ & $1.10 \mathrm{E}-03$ \\
\hline 30 cycles & & & & \\
\hline 1 minute & 2.30E-03 & 4.40E-03 & 8.00E-03 & $1.30 \mathrm{E}-03$ \\
\hline 1 hour & $3.80 \mathrm{E}-04$ & $1.40 \mathrm{E}-03$ & $2.20 \mathrm{E}-03$ & $1.20 \mathrm{E}-03$ \\
\hline 12 hours & $2.60 \mathrm{E}$ & 1.001 & & $9.70 \mathrm{E}-04$ \\
\hline 24 hours & $2.70 \mathrm{E}-04$ & $1.00 \mathrm{E}-03$ & 2.20E-03 & $8.10 \mathrm{E}-04$ \\
\hline 60 cycles & & & & \\
\hline 1 minute & 2.60E-04 & $9.50 \mathrm{E}-03$ & 1.60E-03 & $1.20 \mathrm{E}-03$ \\
\hline 1 hour & $3.20 \mathrm{E}-05$ & $6.40 \mathrm{E}-04$ & 2.10E-03 & $5.20 \mathrm{E}-04$ \\
\hline 12 hours & $8.00 \mathrm{E}-05$ & $5.40 \mathrm{E}-04$ & 2.60E-03 & $5.90 \mathrm{E}-04$ \\
\hline 24 hours & $8.90 \mathrm{E}-05$ & $5.40 \mathrm{E}-04$ & $2.60 \mathrm{E}-03$ & $5.20 \mathrm{E}-04$ \\
\hline
\end{tabular}

It is expected that a disproportionate number of $x=\infty(r=0)$ values from our PMU dataset do not conform within a Rayleigh distribution. These are related to data drops, data 
drifts and other very strong data-related decorrelation events. As such, these values are neglected when fitting the Rayleigh $\sigma$ to the Rayleigh distribution.

\subsubsection{Event Detection Metric}

For $x$ values that conform to the distribution, these are used to derive metrics for power system event detection. Specifically, monitoring $x$ for deviations outside a multiple, $\mathrm{K}$, of the variance can be used as a metric for detecting events defined as

$$
x>\mu(x)+K \operatorname{var}(x)
$$

where $\mathrm{K}$ is an integer multiple. Because $x$ is a fractional number, typically $x<1 \times 10^{-3}$ when in steady-state as shown at the top portion of Table 4.3, Equation 4.8 is normalized by the mean. From Table 4.4, $\mu(x)$ is approximately $1 \times 10^{-4}$. Normalizing Equation 4.8 then becomes

$$
\frac{x}{\mu(x)}>1+K \frac{\operatorname{var}(x)}{\mu(x)}
$$

where values of $\frac{x}{\mu(x)}$ will be, by definition, approximately equal to 1 , and $\frac{\operatorname{var}(x)}{\mu(x)}$ will be approximately $\frac{1}{10}$ (from Table 4.3 and 4.4), both during steady-state. When normalized, $1+K \frac{\operatorname{var}(x)}{\mu(x)}$ is used as the event detection threshold value and Equation 4.9 is the event detection threshold inequality.

To establish this detection threshold value, a setup time over nominal data is first required. To determine a single $r$ value a window size of raw data is analyzed. Recall from Equation 4.3 that $\sigma$ is determined over a window size of $r$ values. Therefore, to determine a valid $\sigma$, a window size of $r$ values are determined which corresponds to two window sizes 
of raw data analyzed. Lastly, to establish steady-state values for $\sigma$, a single minute (3600 cycles) in total of raw data is analyzed before establishing the $\frac{\operatorname{var}(x)}{\mu(x)}$ component.

To show the viability of this detection metric, over a full minute of data containing an event, the normalized $x$ is plotted and the threshold value imposed for a particular $\mathrm{K}$ value and site pair. Prior-event and post-event data is indicated with a dotted blue marker, a dotted red marker indicates the event occurrence (starting at cycle 1100 with a duration of approximately 10 seconds), and the established threshold value indicated with a solid black horizontal line in Figures 4.5, 4.6, and 4.7. Any normalized $x$ which exceeds the threshold value is differentiated with a red 'o' marker. Furthermore, any normalized $x$ which exceeds the threshold value prior and post event lightning strike will be classified as a false-positive and differentiated with a blue ' + ' marker.

To demonstrate this metric over a 60 cycle window size, PMU site Cully is selected from the case study cluster and a set of $\mathrm{K}$ values chosen for testing purposes. Letting $K=10,100$, and 250, from the legend in Figures 4.5, 4.6, and 4.7, the number of normalized $x$ values exceeding the threshold value is 1217,141 , and 6 (respectively) while the false positives are 1019, 106, and 0 . While $K=250$ preserves event detection and includes no false-positives, this value does not represent the optimal $\mathrm{K}$ as it is higher than necessary, adversely affecting event detection. 


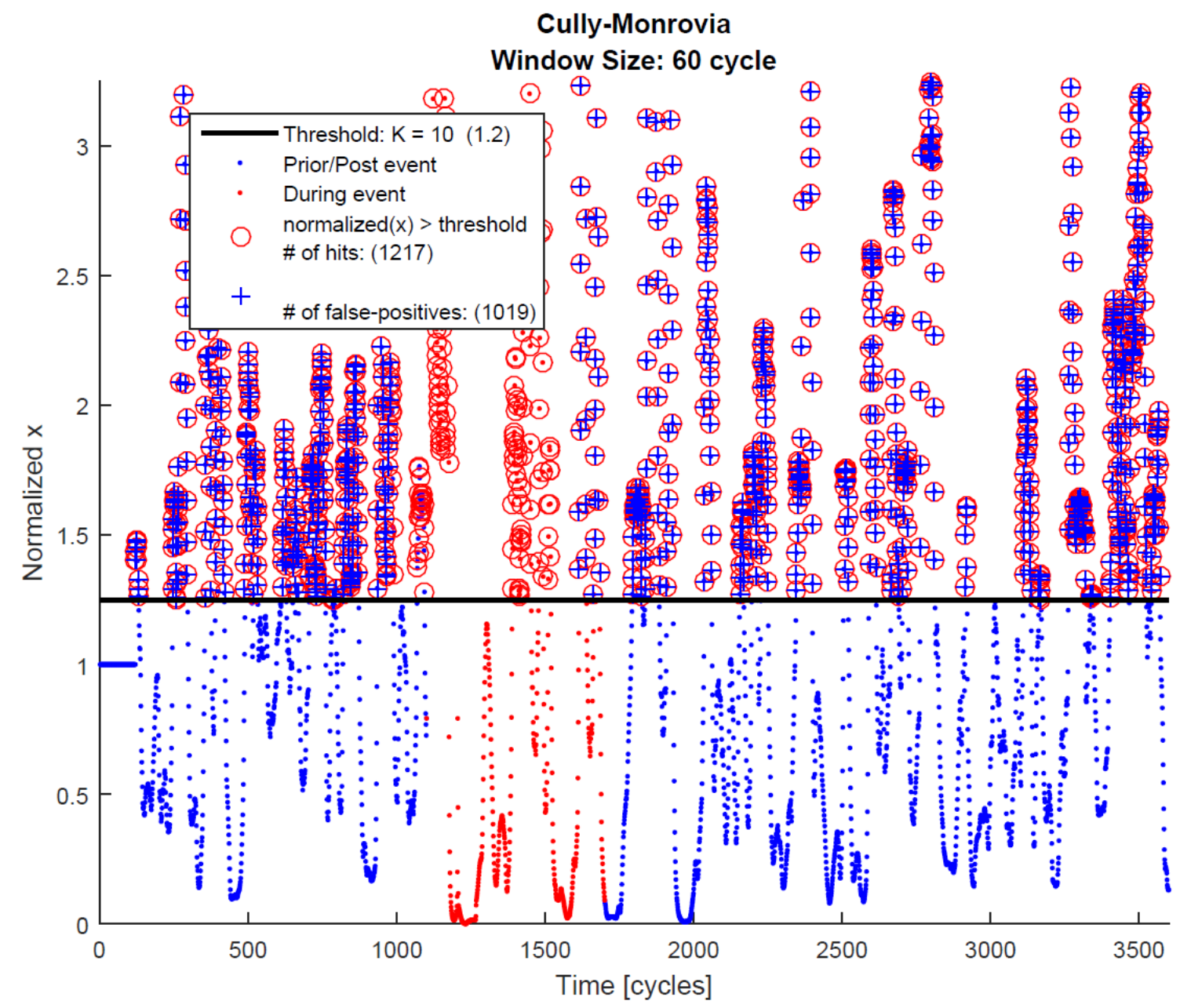

Figure 4.5: Plot of normalized $x$ of $\phi_{+}$over a 60 cycle Ksweep size with threshold value such that $K=10$. The amount of data presented represents approximately 60 seconds of raw data prior/post and including lightning strike occurrence as shown in blue and red dot markers, respectively. Normalized $x$ which exceeds the threshold (shown as solid black horizontal line) are indicated by red 'o' markers. The first row in the legend shows the $\mathrm{K}$ value which establishes the threshold value (shown in parenthetical). The last two row entries in the legend displays the amount of normalized $x$ exceeding the threshold and the classified false-positives (1217, 1019 respectively). 


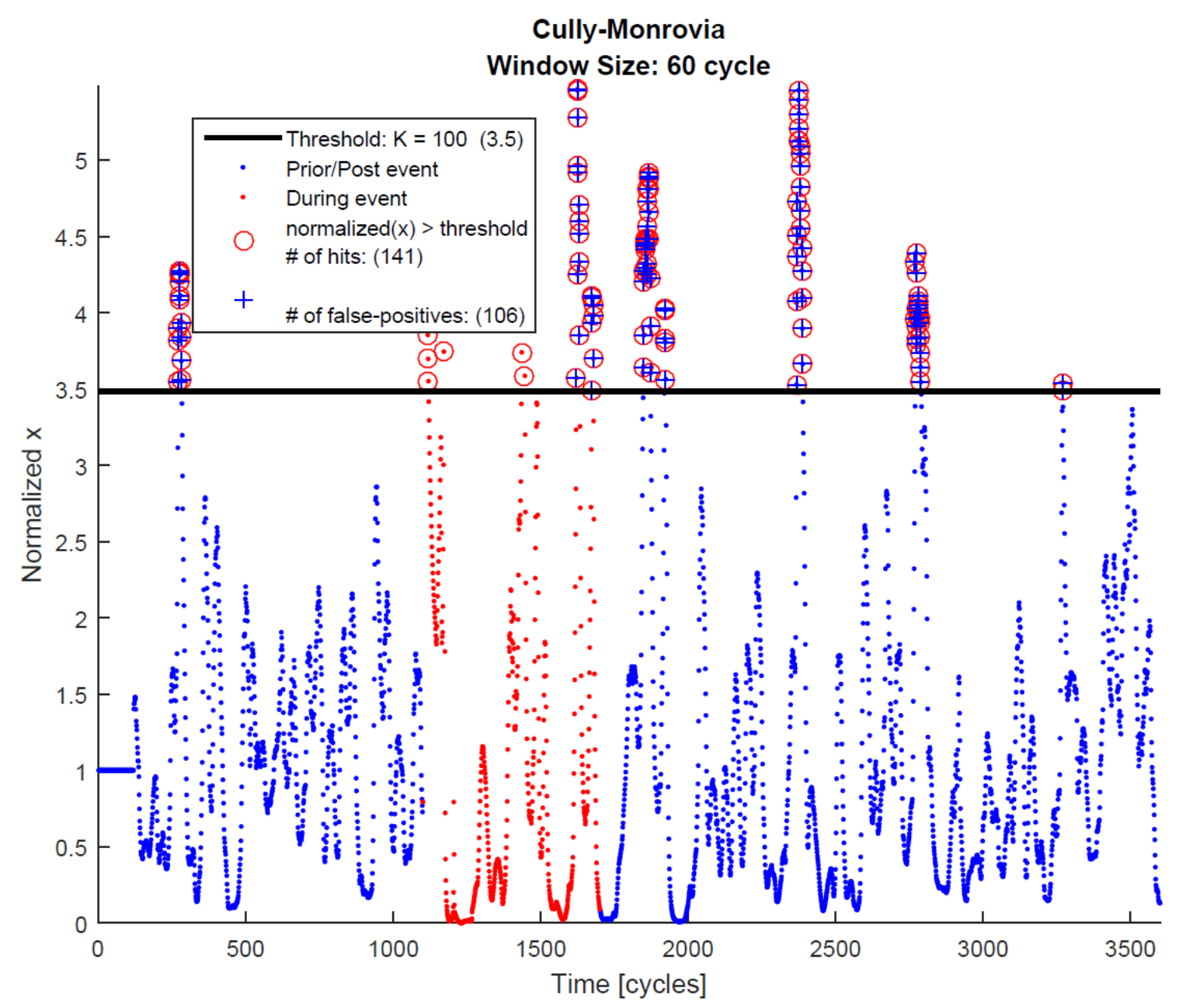

Figure 4.6: Plot of normalized $x$ of $\phi_{+}$over a 60 cycle window size with threshold value such that $K=100$. With this K value, 141 entries exceed the threshold value with 106 false-positives (some entries not shown), implying that this $\mathrm{K}$ is too sensitive, flagging many false-positives. 


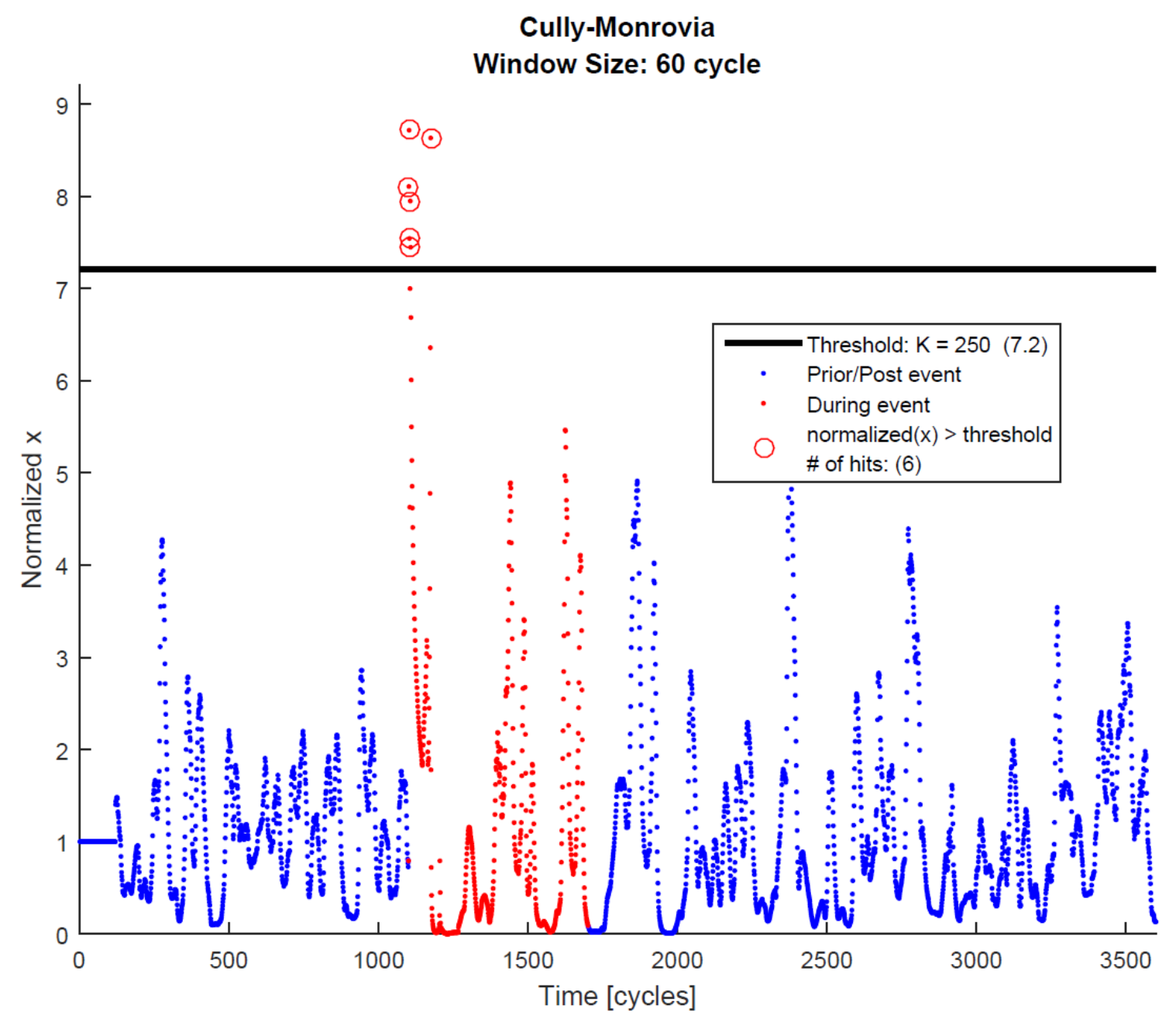

Figure 4.7: Plot of normalized $x$ of $\phi_{+}$over a 60 cycle window size with threshold value such that $K=250$. With this $\mathrm{K}$ value, six entries exceed the threshold value (some entries not shown) with no false-positives, implying that this $\mathrm{K}$ value securely detects this lightning strike occurrence. It is not, however, the optimal $\mathrm{K}$ as it is higher than necessary, adversely affecting event detection.

By sweeping through a range of $\mathrm{K}$ values, the sensitivity of the threshold value to event detection and false-positive classification is investigated. Table 4.5 shows this sweep over a range of $\mathrm{K}$ values. As expected, a lower $\mathrm{K}$ flags for more false-positives than a higher $\mathrm{K}$. Noting this sensitivity, the problem now becomes selecting an optimal $\mathrm{K}$ which minimizes false-positives while adequately preserving detection capabilities. 
Table 4.5: Sweep of $\mathrm{K}$ values to determine amount of normalized $x$ exceeding threshold and classified false-positives (the latter shown in parentheses).

\begin{tabular}{|c|c|c|c|c|}
\hline $\begin{array}{c}\mathrm{K} \\
\text { (based on } \\
15 \text { cycles) }\end{array}$ & Echart & Saul & Washington & Cully \\
\hline 1 & $1804(1531)$ & $1692(1411)$ & $1698(1424)$ & $1715(1474)$ \\
\hline 5 & 1498 (1261) & 1325 (1084) & 1349 (1119) & 1311 (1102) \\
\hline 10 & 1149 (939) & $956(755)$ & $918(734)$ & $913(742)$ \\
\hline 25 & $471(335)$ & $283(201)$ & $237(145)$ & $249(150)$ \\
\hline 50 & $119(63)$ & $39(21)$ & $29(13)$ & $39(14)$ \\
\hline 100 & $3(2)$ & $0(0)$ & $0(0)$ & $0(0)$ \\
\hline $\begin{array}{c}\mathrm{K} \\
\text { (based on } \\
30 \text { cycles) }\end{array}$ & & & & \\
\hline 1 & $1742(1471)$ & 1560 (1269) & $1532(1276)$ & 1537 (1317) \\
\hline 5 & 1603 (1340) & $1247(993)$ & $1022(815)$ & $1038(859)$ \\
\hline 10 & $1451(1204)$ & $929(721)$ & $641(465)$ & $658(539)$ \\
\hline 25 & $1033(845)$ & $386(269)$ & $129(76)$ & 177 (106) \\
\hline 50 & $590(451)$ & $80(47)$ & $10(3)$ & $25(8)$ \\
\hline 100 & 202 (116) & $6(1)$ & $0(0)$ & $0(0)$ \\
\hline $\begin{array}{c}\mathrm{K} \\
\text { (based on } \\
60 \text { cycles) }\end{array}$ & & & & \\
\hline 1 & $1617(1411)$ & $1445(1165)$ & 1387 (1167) & 1519 (1277) \\
\hline 5 & 1549 (1348) & 1134 (921) & $1183(985)$ & 1380 (1159) \\
\hline 10 & 1479 (1282) & $841(674)$ & 1007 (833) & 1217 (1019) \\
\hline 25 & 1291 (1104) & 274 (199) & $609(487)$ & 837 (678) \\
\hline 50 & $947(788)$ & $86(60)$ & $221(173)$ & 398 (310) \\
\hline 100 & $474(366)$ & $3(0)$ & $22(11)$ & $141(106)$ \\
\hline
\end{tabular}

For this particular event, in order to eliminate the event detection threshold inequality (given in Equation 4.9) from evaluating true due to false-positives, the threshold must be set higher than the maximum normalized $x$ value pre-event or post-event. This value, referred to from here on as the maximum false-positive threshold value (MFPTV), is determined over data void of any data-related events, for all PMUs paired with Monrovia over a 60 cycle window size. Figure 4.8 indicates these values with a blue ' + ' marker. For each PMU pair, 
taking the ceiling of the MFPTV signifies the lowest allowable value the threshold should be set to in order avoid erroneously flagging normalized $x$ values as indicative of a power system event occurrence.

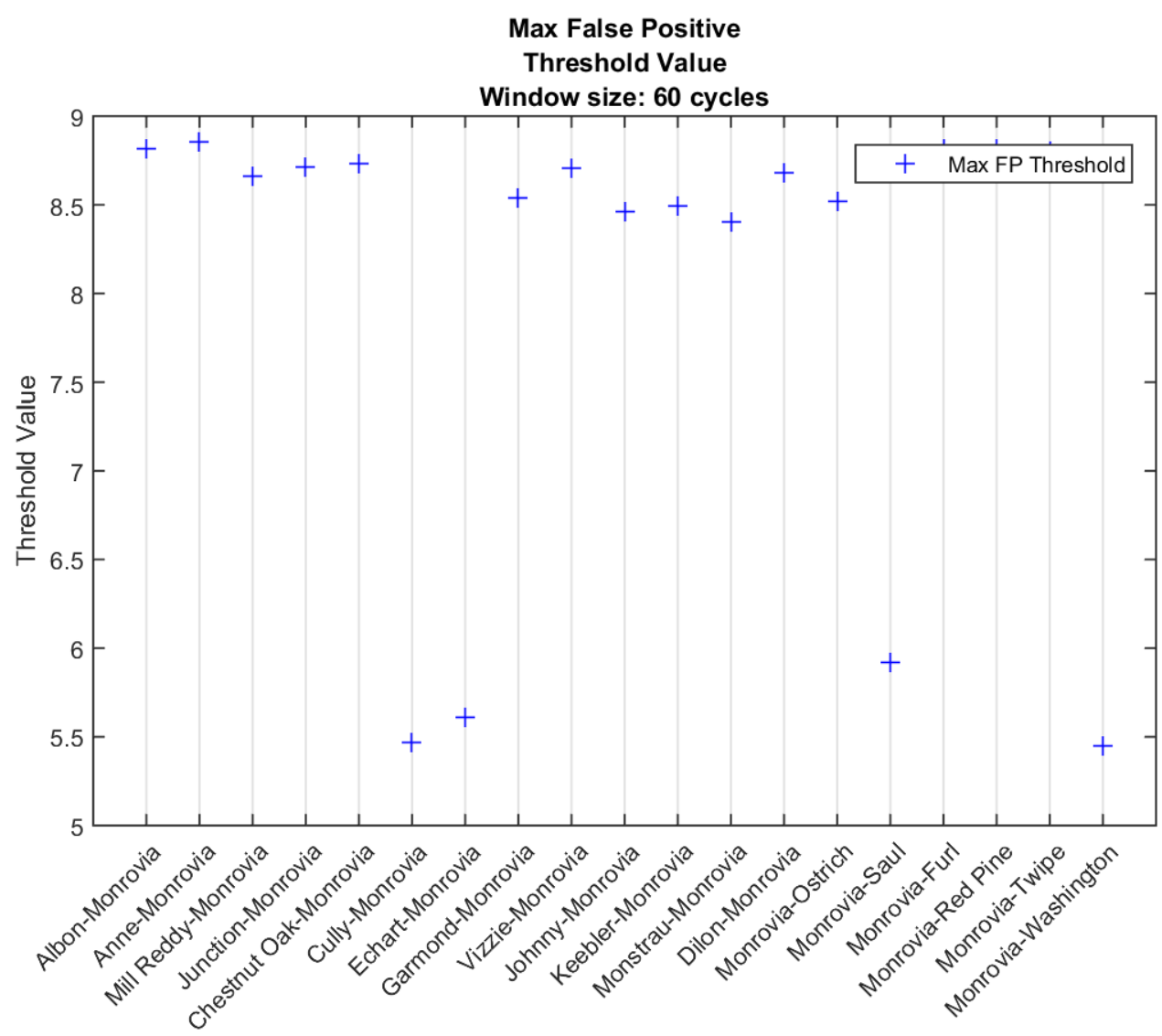

Figure 4.8: Maximum false-positive threshold value (MFPTV) for all PMUs paired with Monrovia during a minute of data containing the lightning strike occurrence. For a particular PMU, in order to avoid having the event detection threshold inequality evaluate true during normalized $x$ values not associated with the event, the threshold should be set slightly higher than the value indicated with a blue ' + ' marker. These values were determined over data void of any data-related issues, and, when analyzing normalized $x$ over a 60 cycle window size.

In a similar fashion, in order to preserve event detection, the event detection threshold inequality must evaluate true at least once during the event. This value, referred to from here on as the maximum true-negative threshold value (MTNTV), is determined for all PMUs paired with Monrovia over a 60 cycle window size. Figure 4.9 indicates these values with a 


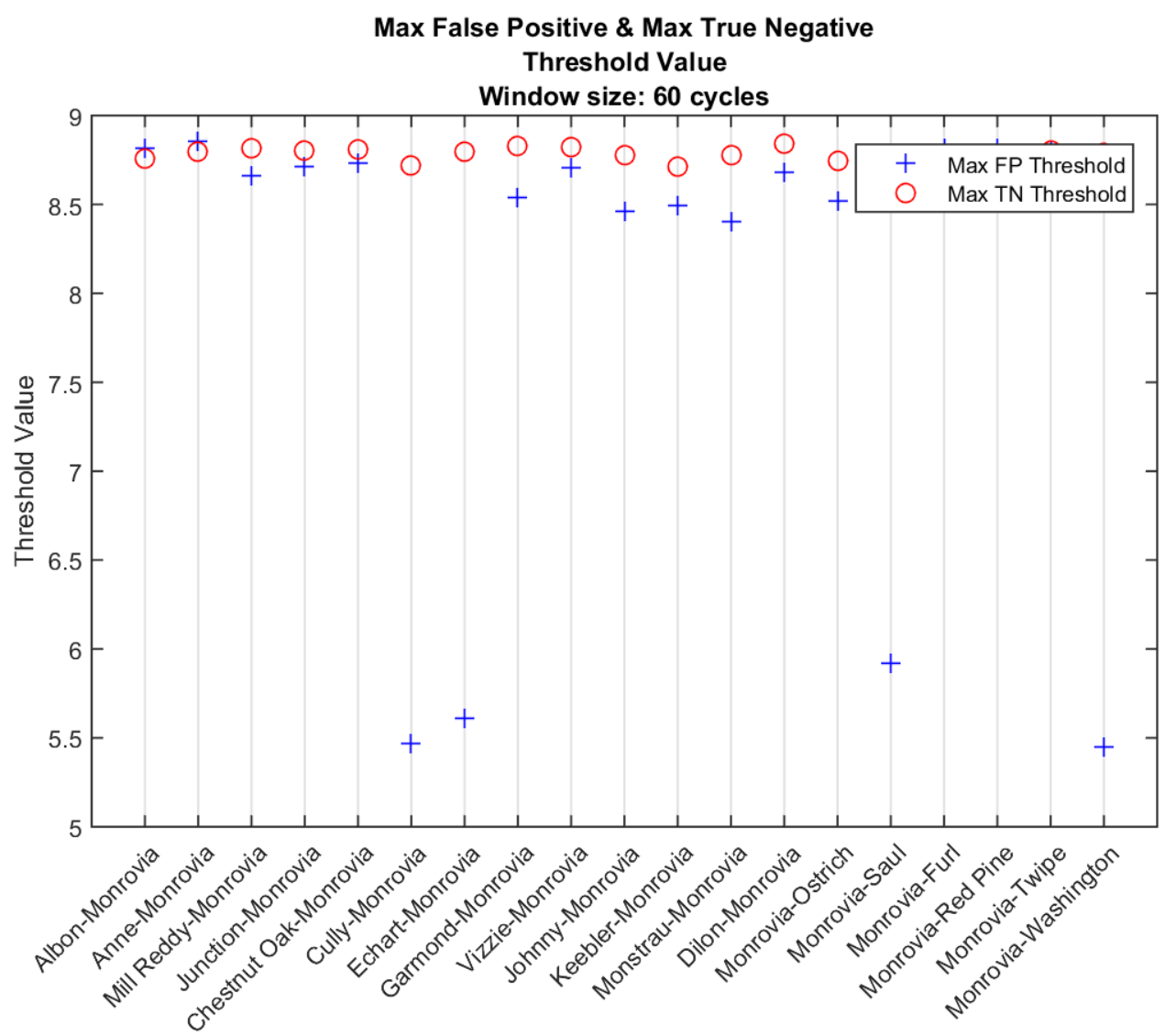

Figure 4.9: Maximum true-negative threshold value (MTNTV - shown with red 'o' markers) imposed on maximum false-positive threshold value (MFPTV - shown with blue '+' markers) for all PMUs during a minute of data containing an event. For a particular PMU, in order to preserve event detection, the threshold should not exceed this value.

red 'o' marker and imposes them on Figure 4.8. For each PMU pair, the MTNTV signifies the largest value the threshold can be set to in order to detect normalized $x$ values during the event occurrence.

The MFPTV and MTNTV will be used in Chapter 4.8 to justify which PMUs to cluster. 


\subsection{Data Events}

Given that higher-level applications may become reliant on PMU-generated data, it is of absolute importance to ensure and maintain integrity of incoming data. In this respect, computation of $r$ can be impacted by occurrences of two known types of data events. These include data 'drop outs' in which data streams will produce a ' 0 ' value when deemed unreliable, and 'stuck at' data issues in which case a PMU produces a repeated value. The latter occurs when a PMU losses synchronicity with the GPS system. These data issues have been dubbed as data drops and data drifts (respectively). These issues must be contended with in order to avoid insecure operations from occurring due to misinformation being operated on erroneously. A metric to handle these known issues has been devised and is discussed in Chapter 5.1.

To explain how correlation is impacted when data issues are encountered, the visual structure can be observed. Figure 4.10 provides a correlation visual for $\phi_{+}$when data issues are being read into the PDC engine. Characteristics of data drop issues include entire rows and columns showing inconclusive correlation $(r=0)$. Similarly, data drift issues can be characterized by blacked out rows and columns with a null symbol. This occurs when the standard deviation of either one or another PMU, $\sigma_{X}$ or $\sigma_{Y}$, goes to zero due to repeated values being reported. With $\sigma_{X}$ and $\sigma_{Y}$ in the denominator of the Pearson correlation coefficient, Equation 4.1, $r$ becomes undefined. 

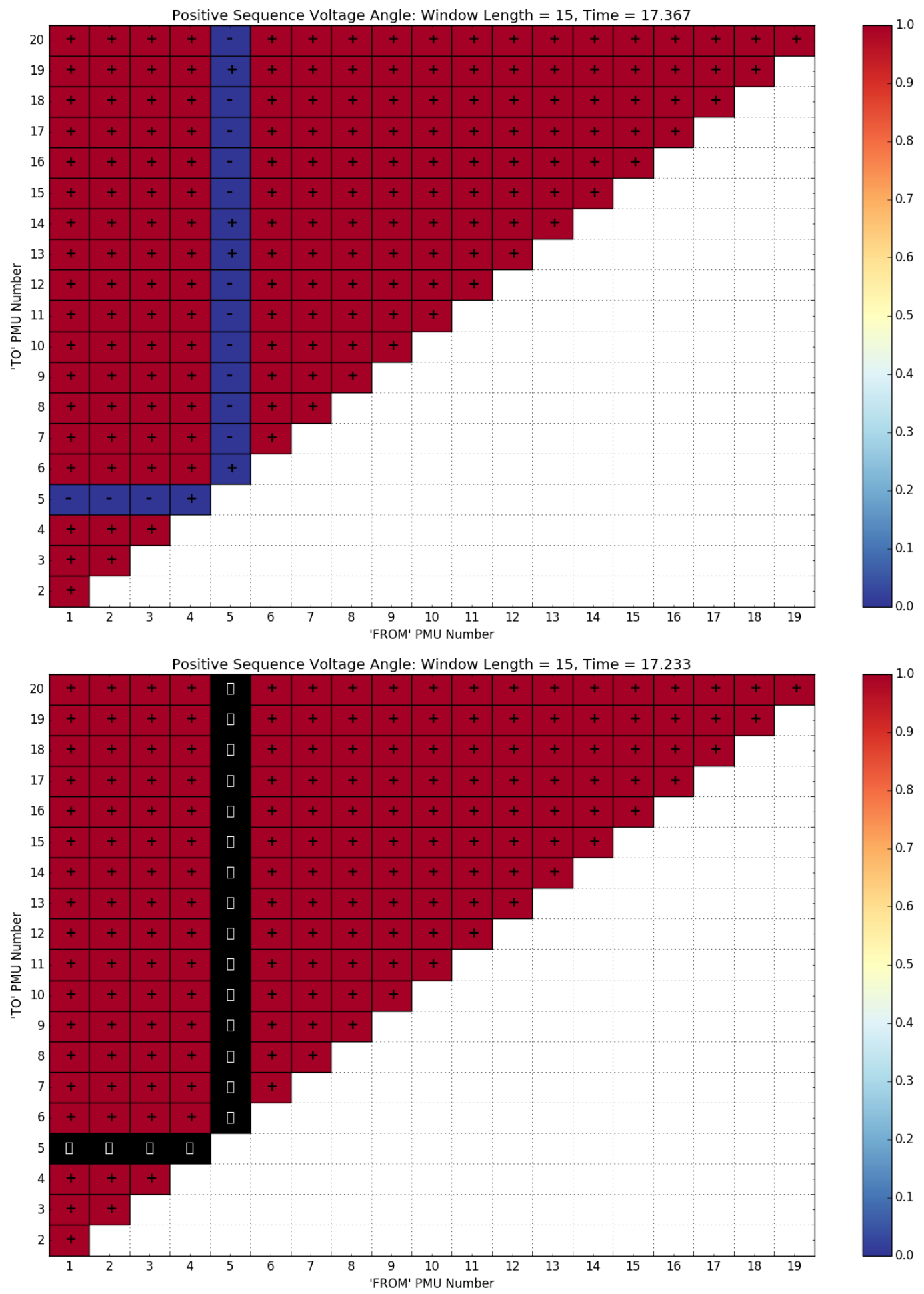

Figure 4.10: Visual structure of correlation coefficients during a data drop (upper) and data drift (lower). Correlation coefficients portray signature characteristics of data event occurrences. Characteristic of data drops, entire rows and columns of inconclusive correlation $(r=0)$ as shown in the upper figure due to a data drop at PMU Number 5. Characteristic of data drifts, entire blacked out rows and columns shown in the lower figure due to a data drift at the same PMU Number. 


\subsection{Power System Events}

In regards to power system events, $r$ has been observed to become decorrelated regressing from a 'very highly' correlated state to a lower state of correlation. The severity of decorrelation depends on two factors; electrical proximity and window size. For instance, electrically close sites regress to 'moderately' correlated within a time frame approximately equal to

$\frac{1}{3}$ of the window size when using 15 and 30 cycle window sizes and approximately $\frac{1}{2}$ of the window size when using a 60 cycle window size. Comparing with electrically-far sites, correlation regression of $r$ to 'moderately' correlated happens in approximately $\frac{1}{2}$ of the window size when using 30 and 60 cycle window sizes but will never leave a 'very highly' state when using a window size of 15 cycles.

To explain how correlation is impacted during an event, the visual structure can again be used. Figure 4.11 provides a correlation visual of $\phi_{+}$over a 30 cycle window size, qualitatively highlighting an event occurrence. Characteristics of this include varying levels of poorly correlated rows and columns. Most notable here is PMU Number 1, representing PMU site Monrovia, slowly becoming decorrelated with the rest of the system, thereby indicating a sudden change in $\phi_{+}$. In addition, PMU Number 2, representing PMU site Echart, exhibits similar behavior but to a lesser extent. This indicates that the event happened between these two sites. It can be seen that the organization of PMUs will produce electrically coherent zones. As a result, the visualization will naturally cluster, thus benefiting ease of analysis. This observation will be capitalized on by strengthening the case for which PMUs are selected to be included in the clustering scheme. 


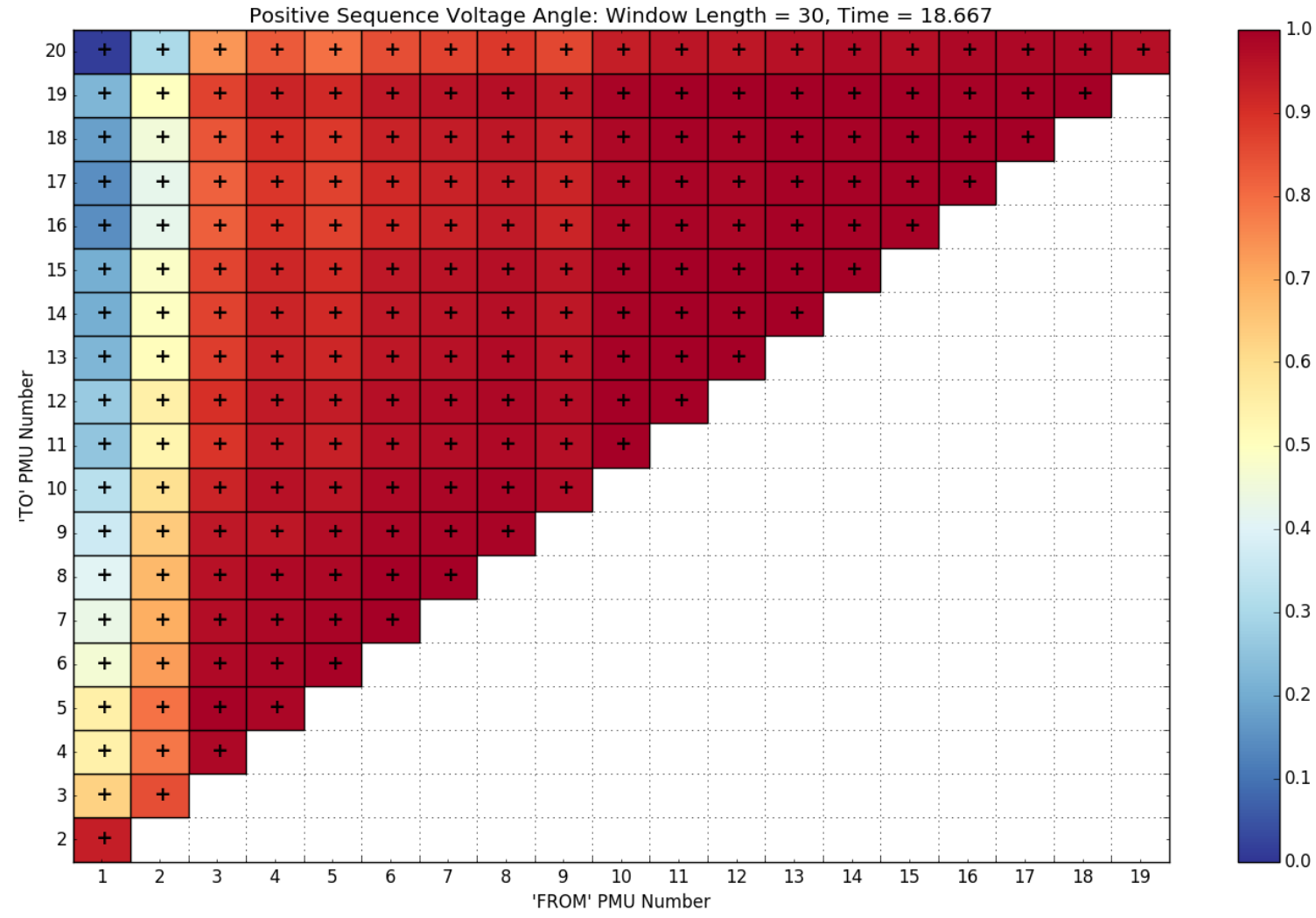

Figure 4.11: Visual structure during an event. Correlation with the sites experiencing an event most strongly (in this instance PMU Number 1 - Monrovia and PMU Number 2 - Echart) have become decoupled, indicating an event has occurred between these sites. Characteristics of an event are rows and columns exhibiting varying levels of poor correlation. When sites are arranged by electrical distance (w.r.t. the event), a gradient of correlation ( $r$ decreases as electrical distance increases) emerges within the visual structure. 


\subsection{Cluster Size}

To choose which PMUs are ideal for monitoring, the MFPTV and MTNTV are used to eliminate unnecessary PMUs and select those that are least likely to flag false-positives. It can be observed from Figure 4.9 that there exist some MFPTV > MTNTV. This implies that this PMU pair will always report an event due to a false-positive. As such, these PMU pairs should be excluded from being clustered. Figure 4.12 excludes these PMUs in order to observe which remaining PMU pairs may suitable for clustering.

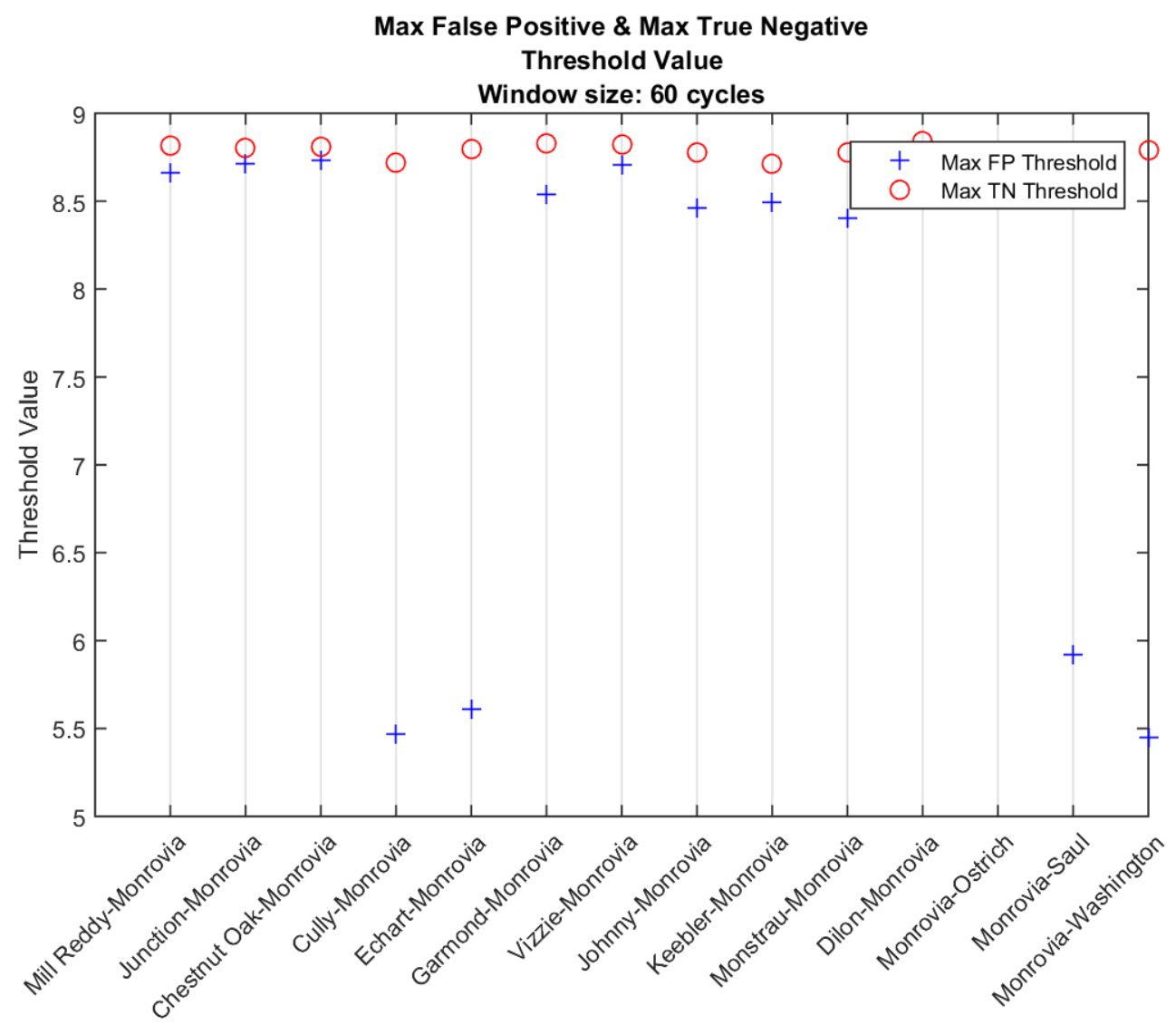

Figure 4.12: PMUs with MFPTV > MTNTV, over a 60 cycle window size, are excluded as these will always be flagged by false-positives.

While the number of PMUs have been reduced, even more can be excluded from being 
clustered. Some PMU pairs exhibit small MFPTV. These are most suitable for monitoring as they decrease the probability of flagging for false-positives and better preserve event detection. Figure 4.13 focuses on these PMUs. Since large window sizes will be used to detect events and small window sizes to confirm they are not data-related, this approach to determining which PMUs to cluster is only performed for a large window size. It should be noted that these PMUs represent the case-study cluster and, due to the reasons above, justify their inclusion into the cluster.

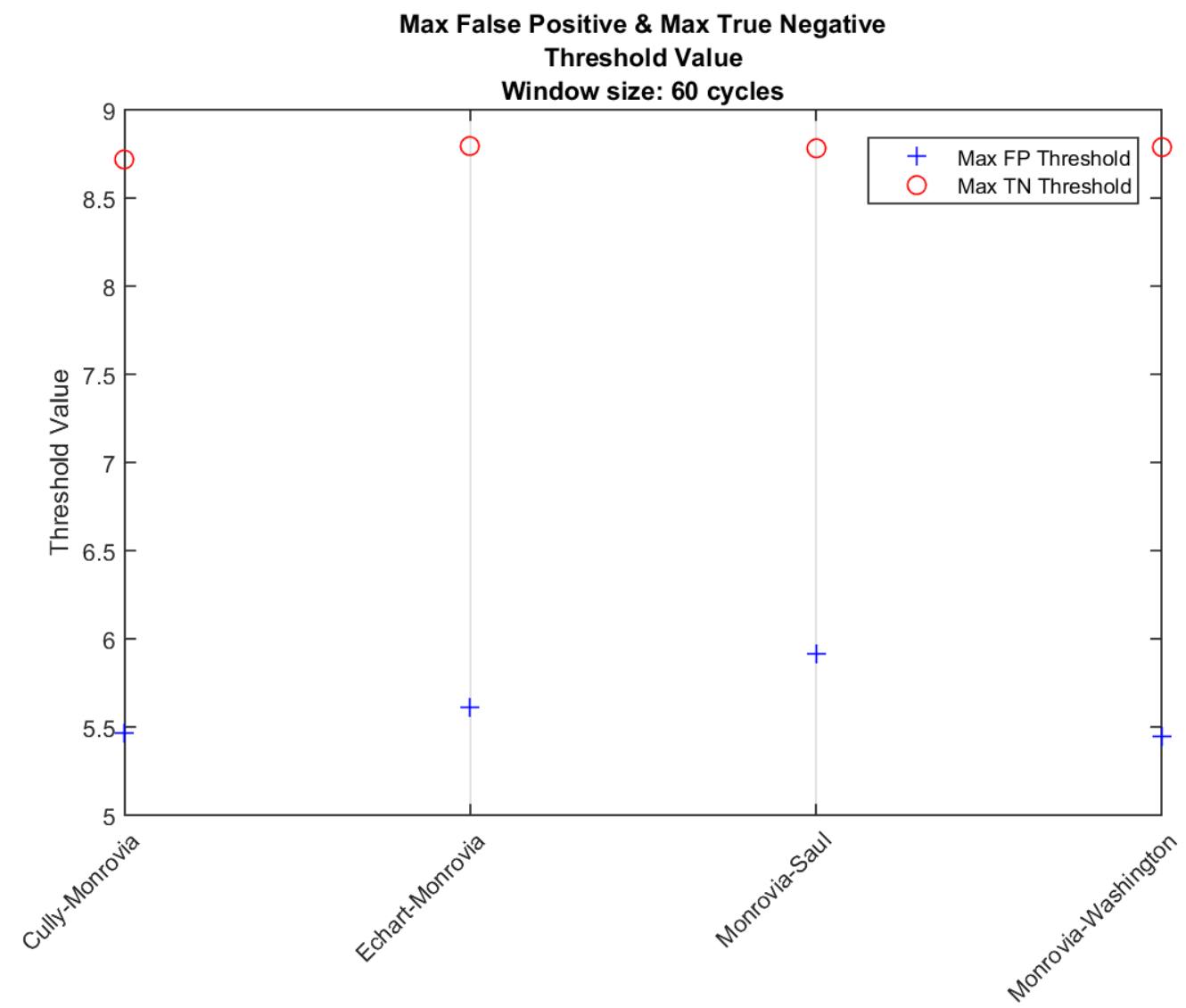

Figure 4.13: PMUs with MTNTV > MFPTV and with lowest MFPTV are shown. These PMUs represent those found in the case-study cluster. 


\subsection{Cluster Content}

As a recap, a cluster is comprised of a subset of PMU sites containing a combination of electrically near and far sites with respect to the site experiencing the event. Clusters containing only 'near' PMU sites, such as the one shown in Figure 4.14, may not be sufficient for properly detecting the event as $r$ will remain highly correlated given the similar responses that adjacent sites will exhibit. Additionally, clusters possessing all far PMU sites, such as the one shown in Figure 4.15, will led to inadequate event detection as correlation will vary having some strongly and weakly correlated $r$.

To address this, clusters are comprised of a combination of both near and far sites. This clustering scheme will be investigated and justified in Chapter 5. This will be shown to be most successful at detecting events; far PMU sites begin to decouple easily due to large electrical distance allowing for detection of event occurrences while near PMU sites can confirm that the event has occurred based on its near $r$ value since they experience nearly the same degree of impact. 

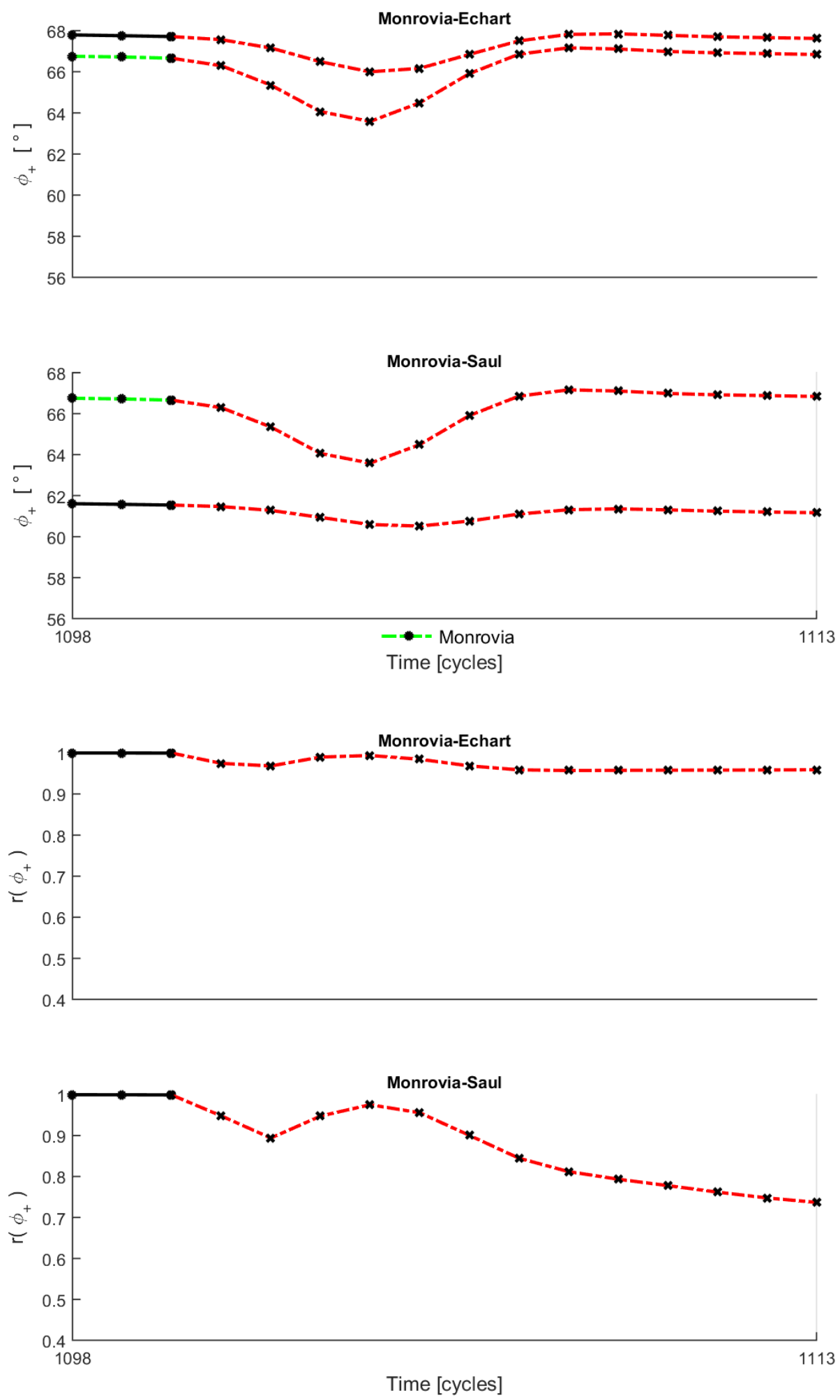

Figure 4.14: The response to an event of an all near cluster containing two electrically close PMU sites. (Top) Phase angle comparison. (Bottom) Impact on $r$ with an all near-cluster. Notable is site Echart located so electrically close to Monrovia that correlation is not adversely affected. 

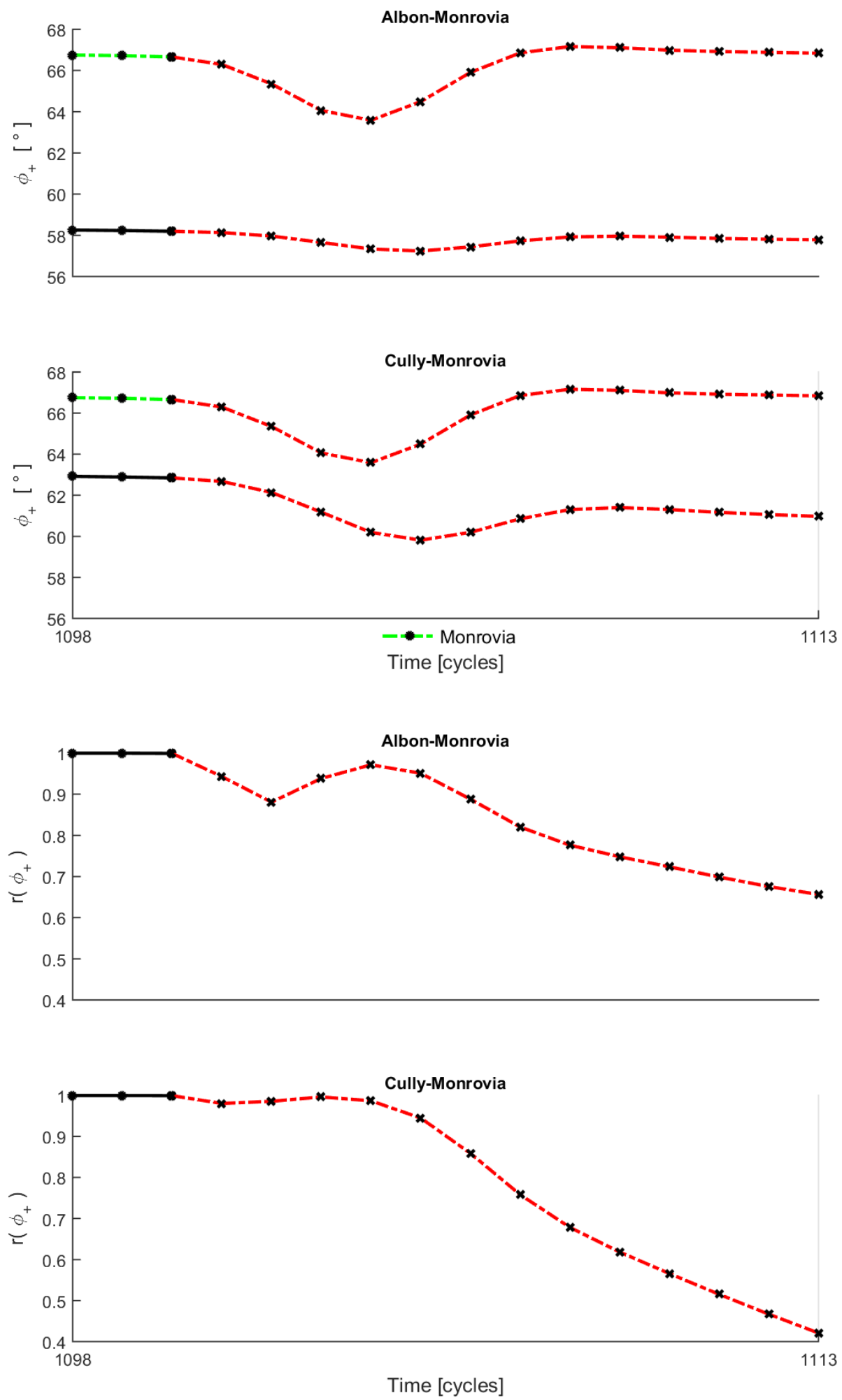

Figure 4.15: The response to an event of an all far cluster containing two electrically-far PMU sites. (Top) Phase angle comparison. (Bottom) Varying values of $r$ with an all far-cluster. 


\section{$5 \quad$ Results \& Analysis}

To facilitate in development of an event detection algorithm, BPA has provided an event log summarizing locations, times, and causes of all known events that occurred during this time frame. The amount of events capturing during this time totals a count of five. Of these five events, three of them were lightning strike occurrences at or near site Monrovia. The other two events, one due to foreign trouble and the other a lightning strike occurrence, occurs at PMUs which were not included in the dataset. Due to the limited availability of event data, this section focuses on the three lightning strike occurrences near PMU site Monrovia.

Analysis in this section is centered around the particular case study cluster discussed in Chapter 4.8 and depicted in Figure 4.4. The $V_{+}$and $\phi_{+}$time domain profiles of this PMU cluster are shown in Figure 5.1 over a 15 cycle window size with the event differentiated from nominal data (shown by green-dotted, o-marked portions) by red-dotted, $\mathrm{x}$-marked portions. The cluster has been arranged by ascending order of electrical distance (relative to the event). This window size is selected to best depict the subtle transient nature of the disturbance. 

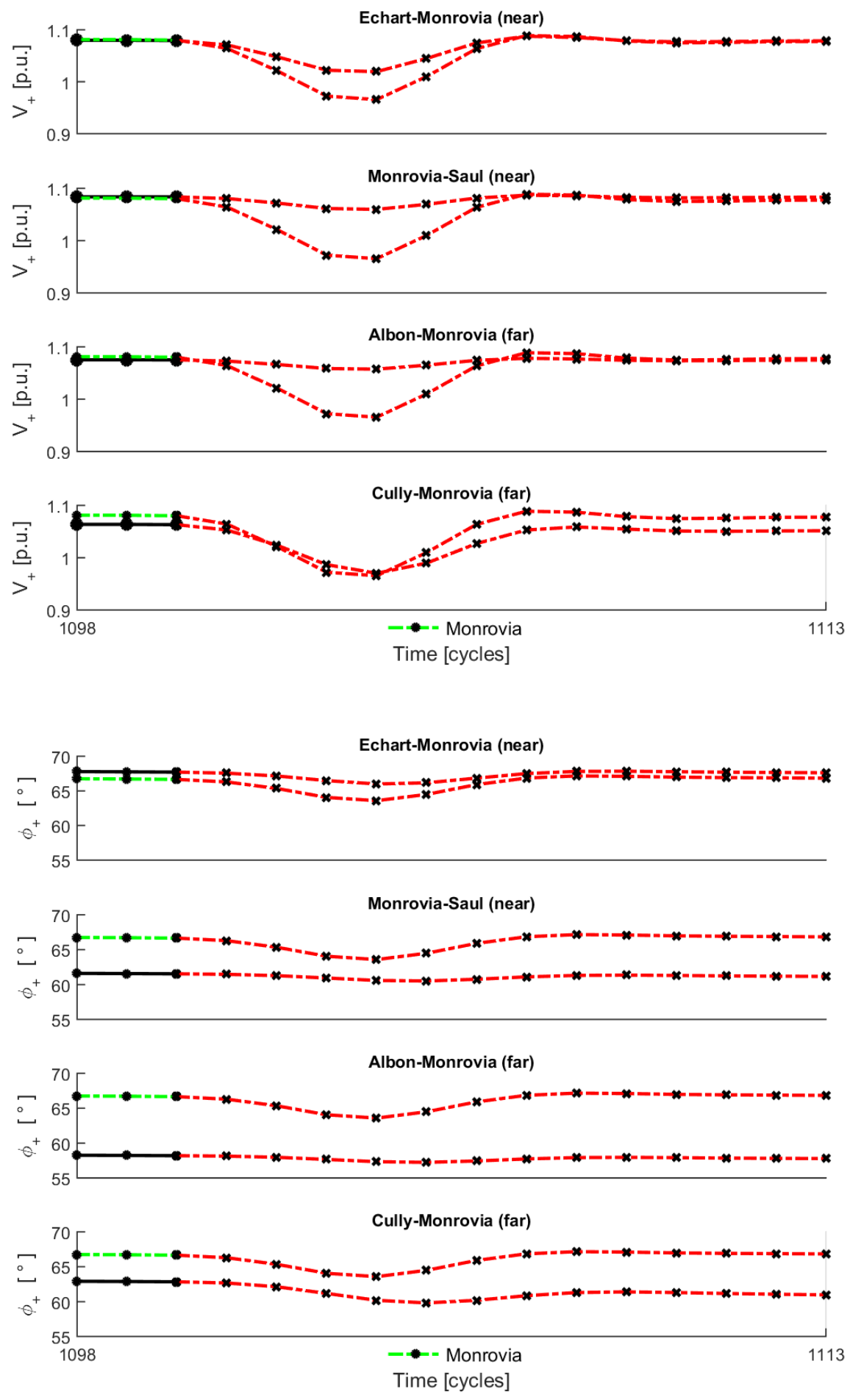

Figure 5.1: Time domain plot of $V_{+}$and $\phi_{+}$during lightning strike as captured by case study PMUs. 


\subsection{Detecting Data Errors and Power System Events}

As mentioned previously, integrity of PMU data must be preserved in order to ensure reliable operations are performed.

Modifying the Monrovia data stream to contain a 3-cycle data drop (shown at top of Figure 5.2), the adverse impact at the correlation layer produced by such an occurrence is depicted on the bottom of Figure 5.2 and demonstrates the capability of detecting this type of data error.

Additionally, when PMUs consecutively produce a constant value (i.e. a data drift), correlation is also adversely affected. To exemplify this type of data event, 20 cycles of data were duplicated within the Monrovia data steam. Figure 5.3 demonstrates the impact on correlation when observed with 15, 30, and 60 cycle window sizes (from top to bottom, respectively). When observing $r$ over a 15 cycle window size, Monrovia quickly becomes decorrelated from the rest of the system transitioning from 'very highly' to 'low' correlation over nearly a single window size. After an entire window size, correlation becomes undefined (shown by the absence of $r_{1035}$ ). Correlation remains 'very highly' correlated for the larger window sizes. However, correlation barely deviates from $r=1$ when approximately $\frac{1}{4}$ of the entries are constant for a 60 cycle window size.

Recall the denominator term of Equation 4.1 will be zero when either $\mathrm{X}$ or $\mathrm{Y}$ does not vary over the window size, $\mathrm{N}$, resulting in $r$ to be undefined. This is the case when observing the absence of the last entry in the correlation layer under a 15 cycle window size. Therefore, smaller window sizes are excellent candidates for detecting these types of 

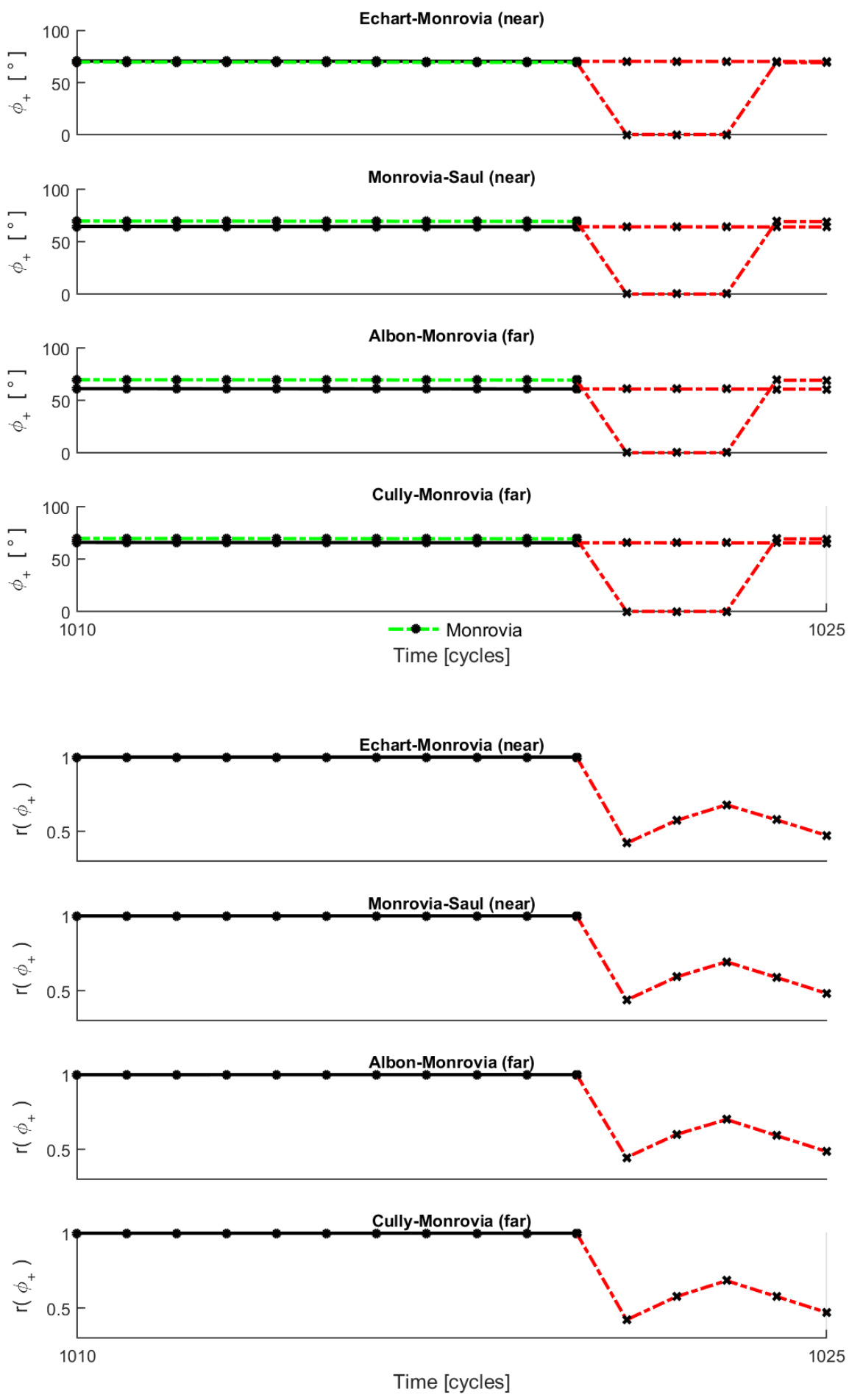

Figure 5.2: (Top) 3 cycle data drop injected into Monrovia data steam. (Bottom) Correlation layer showing sensitivity to data drop occurrence. 
data issues; flagging data streams which exhibit this behavior as a false-positive and not as an indication of an actual event. The visual structure quantitatively depicts these types of occurrences in Chapter 4.10.

In regards to power system events, the case study indicated in Figure 4.4 of Chapter 4.4 will be analyzed. Figure 4.1 depicts the lightning event in the time domain for the Monrovia site and Figure 5.1 portrays the response of the overall cluster. 

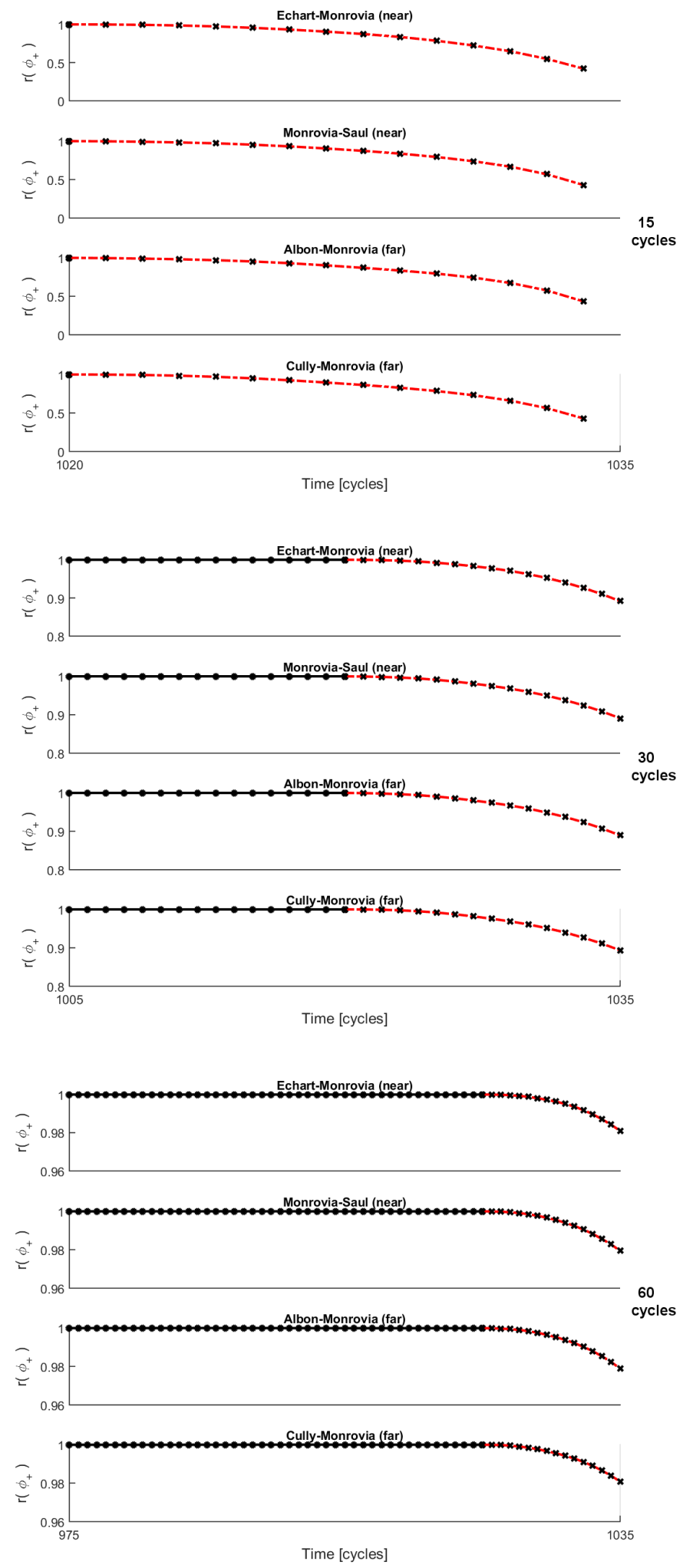

Figure 5.3: Correlation layer during data drift injection into Monrovia data steam. From top to bottom are 15, 30 , and 60 cycle window sizes. Note the absent $r_{1035}$ value for a 15 cycle window size which is otherwise present for the larger window sizes. Note differences in the y-axis scale. 


\subsection{Window Length}

In Section 5.1, varying the window size affected the sensitivity of the algorithm in detecting a data drift event. In this case, a smaller window size may be used to detect these type of data integrity issues, classifying it as a false-positive.

To increase robustness in event detection, varying the window size is a technique for observing the impact on $\mu(x)$ and $\operatorname{var}(x)$ of the case study cluster. Figures 5.4 and 5.5 show how these parameters vary 13 cycles into the event (start of the event indicated by red vertical line). It is observed that as window size increases, both $\mu(x)$ and var $(x)$ decrease.

To quantitatively see this robustness, the correlation visual is used. Figure 5.6 shows how $r$ varies, with window size, 13 cycles into the event. Over larger window sizes the correlation visual is highly correlated during steady-state, represented as all red coordinate squares. During an event, larger window sizes show correlation slowly regressing, as shown in the bottom of Figure 5.6, which indicates an event occurrence. Visuals with smaller window sizes are then used to confirm that this regression in correlation is not due to a data issue but is, in fact, an actual power flow contingency. This demonstrates that using multiple window sizes offers increased robustness in detection of power system events. 

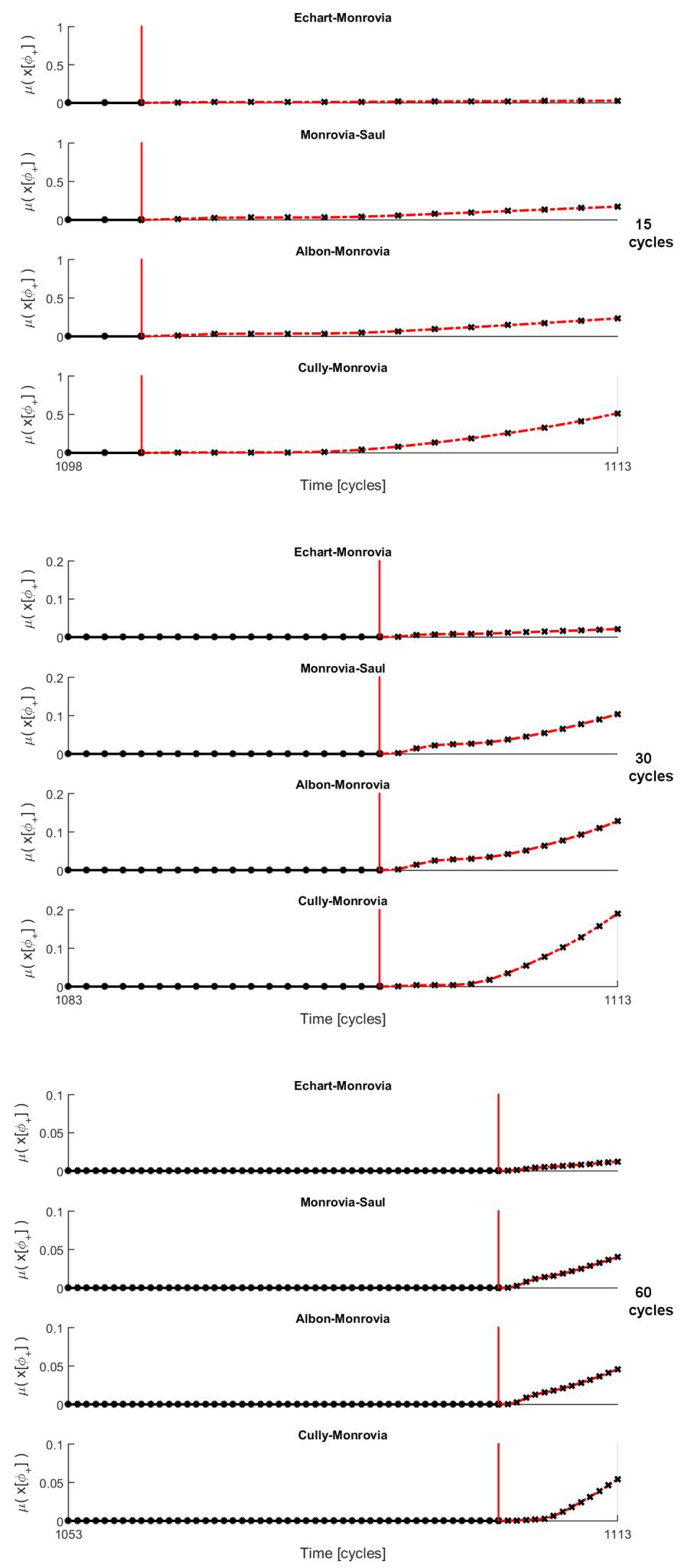

Figure 5.4: $\mu(x)$ of $\phi_{+}$, for the case study PMU cluster, 13 cycles into a lightning event over a 15, 30, and 60 cycle window size. Start of the event indicated by vertical red line. Notable are the changes of scale (y-axis) with window size. 

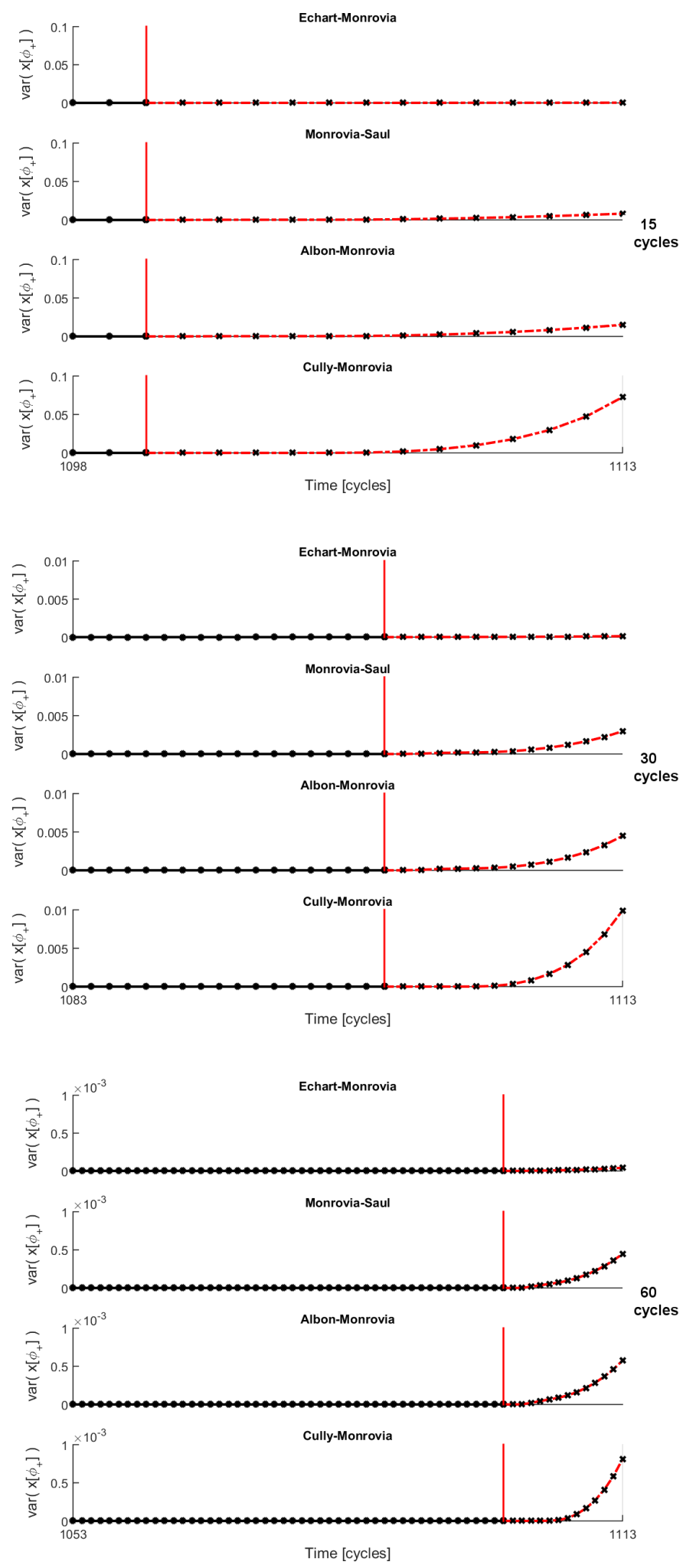

Figure 5.5: $\operatorname{var}(x)$ of $\phi_{+}$, for the case study PMU cluster, 13 cycles into a lightning event over a 15, 30, and 60 cycle window size. Start of the event indicated by vertical red line. Notable are the changes of scale (y-axis) with window size. 

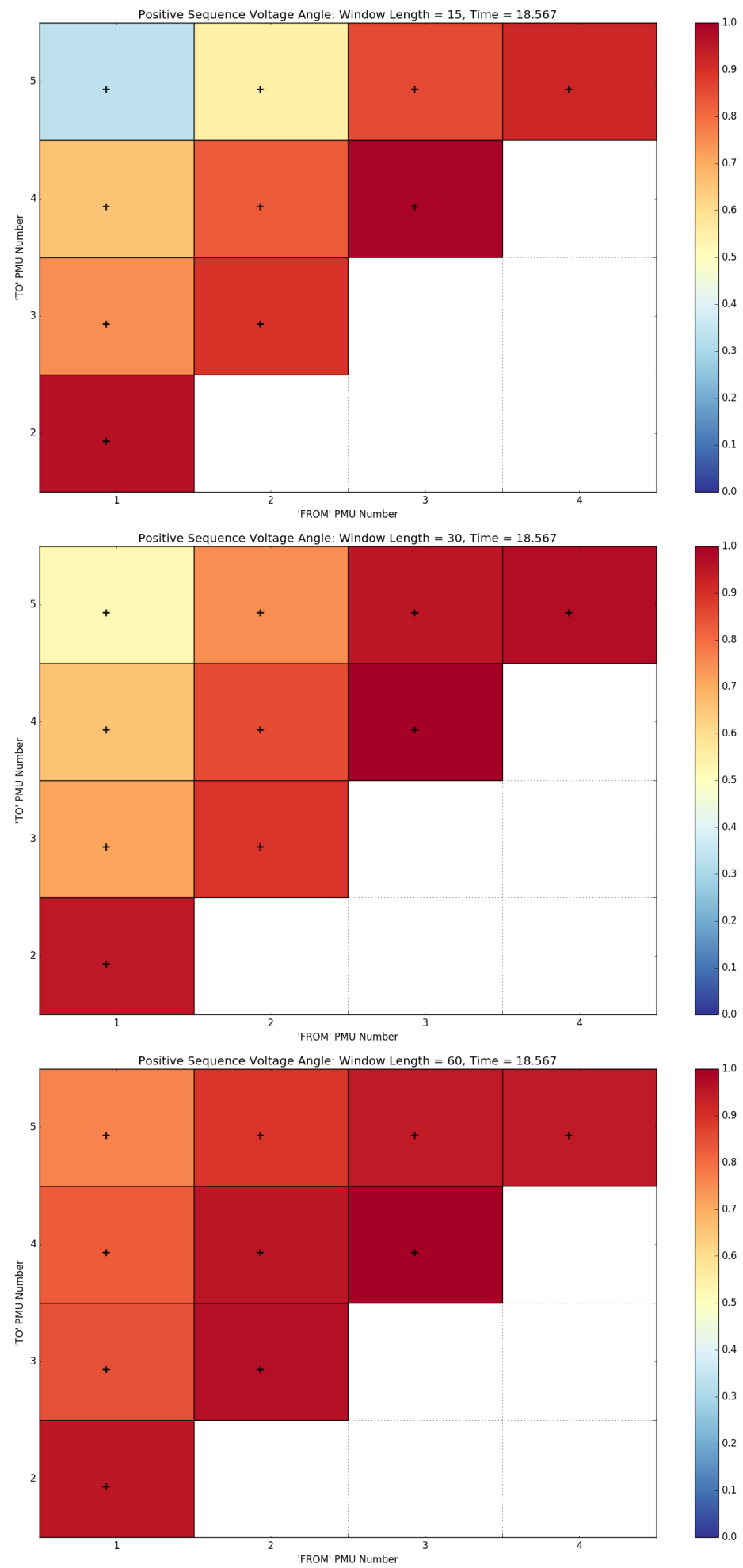

Figure 5.6: Correlation visual of $\phi_{+}$, for the case study PMU cluster, 13 cycles into an event over a 15, 30, and 60 cycle window size. Larger window sizes detect the event occurrence. Smaller window sizes confirm occurrence of an actual power system contingency, rather than a data issue. 
To take full advantage of this robustness, multiple detection algorithms with each using different window sizes should be used to monitor a data stream concurrently. In this fashion, when data events occur they can be detected sooner with smaller window sizes, informing larger window sizes of the data issue. Similarly, when power system events occur they can be detected as such by larger window sizes and confirmed with smaller window sizes. This advantage is only made possible when a minimum of two detection algorithms are used; one using a small window size and the other using a large window size. 


\subsection{Rayleigh Characteristics}

Given the presumed fluctuating nature intrinsic to $V_{+}$, it is expected that $\operatorname{var}(x)$ exhibits more volatility compared to $\phi_{+}$. The difference in scale is stark, $V_{+}$being $1 \times 10^{5}$ times more, as can be observed along the y-axis of $\operatorname{var}(x)$ plots shown in Figure 5.7. As such, analyzing $\phi_{+}$proves more useful for event detection due to the variance exhibiting sensitivity to sudden drastic changes with $\phi_{+}$.

Additionally, Table 4.2 in Chapter 4.5 showed up to $6.44 \%$ of $r$ values are negative for $V_{+}$. Given the above statements about volatility and considerably higher occurrences of negative $r$ entries, analysis of $\mu(x)$ and $\operatorname{var}(x)$ were not performed for $V_{+}$.

When observing the Rayleigh distributions of $\phi_{+}$over nominal data, the distributions (shown in top portion of Figures 5.8, 5.9, and 5.10 for 15, 30, and 60 cycle window sizes, respectively) resemble the expected profile of a general Rayleigh probability density function (PDF). However, when observing 13 cycles into the occurrence of a lightning strike event, $x$ deviates from its previous distribution profile quite drastically, as shown in each bottom portion of the respective figure (distinguished with red ' $x$ ' marker). This rapid occurrence of large outliers is what we use to detect power system events. As a note, both axes on the distribution are held constant for comparative purposes.

To re-enforce understanding of this event detection metric, lets us observe how instances of $x$ outliers in the Rayleigh distribution emerge, as shown at the bottom of Figures 5.8, 5.9, and 5.10. When analyzing $\phi_{+}$between Monrovia and its clustered sites prior to the event, correlation is quantified as very high $(r=1)$ since the system is operating in steady-state. 

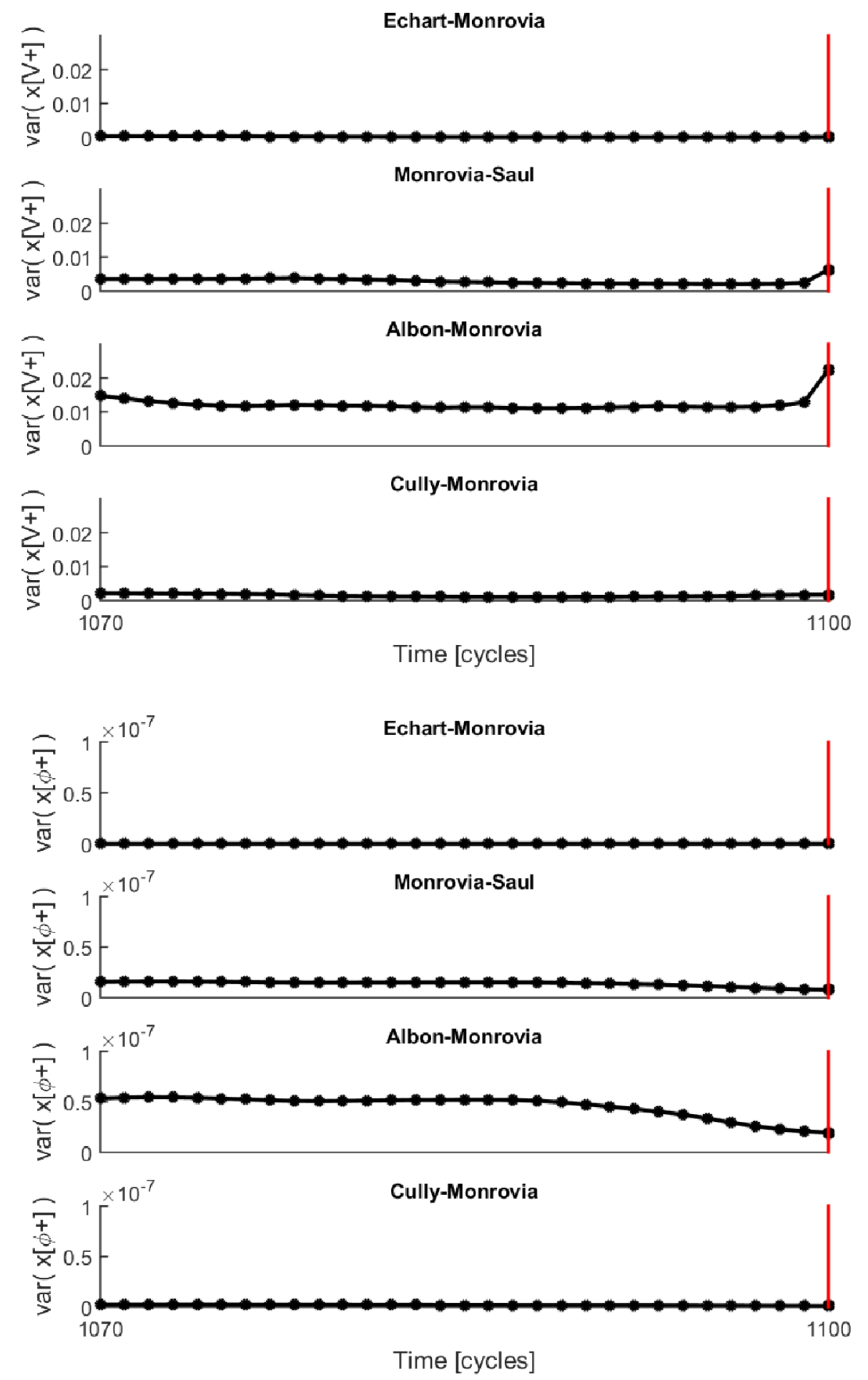

Figure 5.7: $\operatorname{var}(x)$ of $V_{+}$and $\phi_{+}$over 30 cycle window size during steady-state just prior to an event. The difference in $\operatorname{var}(x)$ is several orders of magnitude as shown by the different scales of the y-axes. 
At the instant the lightning strike occurs, a line-to-ground fault takes place near Monrovia, causing a sudden change in real power flow ${ }^{1}$ indicated by the time domain profile of $\phi_{+}$, depicted previously in Figure 4.1 of Chapter 4.2. Recall from Chapter 3.3 the primary reason $\phi_{+}$is selected as the parameter to analyze is because it varies slowly (in contrast to $V_{+}$) and it trends similarly between adjacent PMU sites causing $r\left(\phi_{+}\right)$to be highly sensitive to impulse events that alter real power flow. Observing Equation 4.5,

$$
x=y-1=\frac{1}{|r|}-1
$$

noting that $r\left(\phi_{+}\right)$determines its value, $x$ should remain approximately zero during steadystate. With the sensitive nature of $r\left(\phi_{+}\right)$in mind, a lightning strike event causes an abrupt change in real power flow, and more directly $r\left(\phi_{+}\right)$, which is in fact what is captured and signified by $x$ outliers in the Rayleigh distribution as shown at the bottom of Figures 5.8, 5.9, and 5.10.

During an event, as shown from the bottom of Figures 5.8, 5.9, and 5.10, as window size increases the x-axis decreases. Comparing Figure 5.9 to Figure 5.8, a decrease in scale, as much as $49 \%$, can be seen along the x-axis. Comparing Figure 5.10 to Figure 5.9, a decrease in scale, as much as $38 \%$, can be seen along the $\mathrm{x}$-axis. This implies that, with larger window sizes, the sensitivity of the algorithm decreases when analyzing $\phi_{+}$since $x$ will exhibit small variations, compared to smaller window sizes, which will exhibit much larger variations with $x$.

\footnotetext{
${ }^{1}$ The colloquial phrase 'real power flow' is used in this thesis, because this is the common usage in power engineering circles, as opposed to the grammatically correct phrase 'real power.'
} 

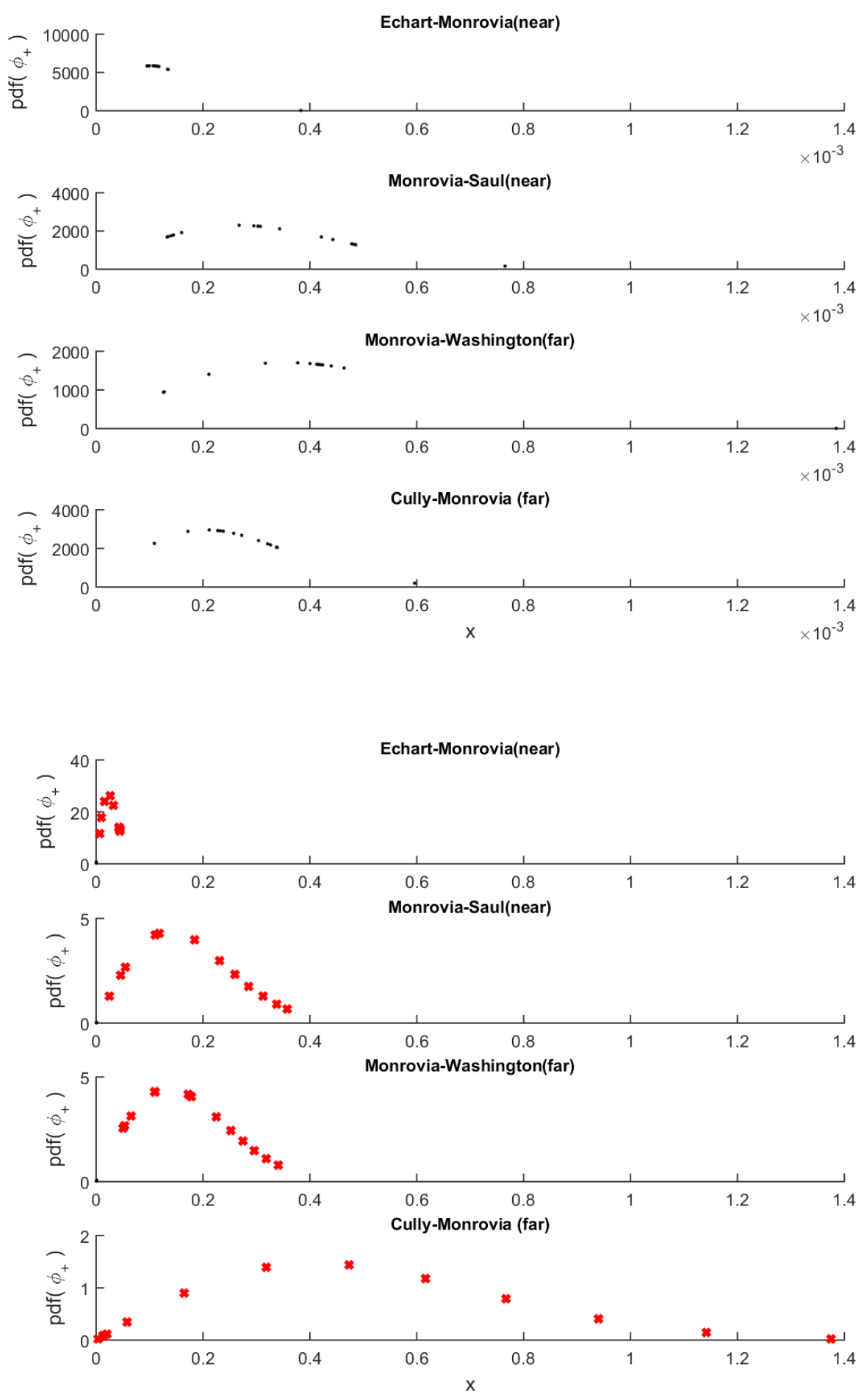

Figure 5.8: Rayleigh distribution of $\phi_{+}$over 15 cycle window size with $\mathrm{x}$-axis held constant for comparative purposes. (Top) Steady-state profile of Rayleigh prior to lightning strike. (Bottom) 13 cycles into a lightning event causing $x$ to deviate from steady-state. Note the change of scale of the $\mathrm{x}$-axis from steady-state data to event occurrence. 

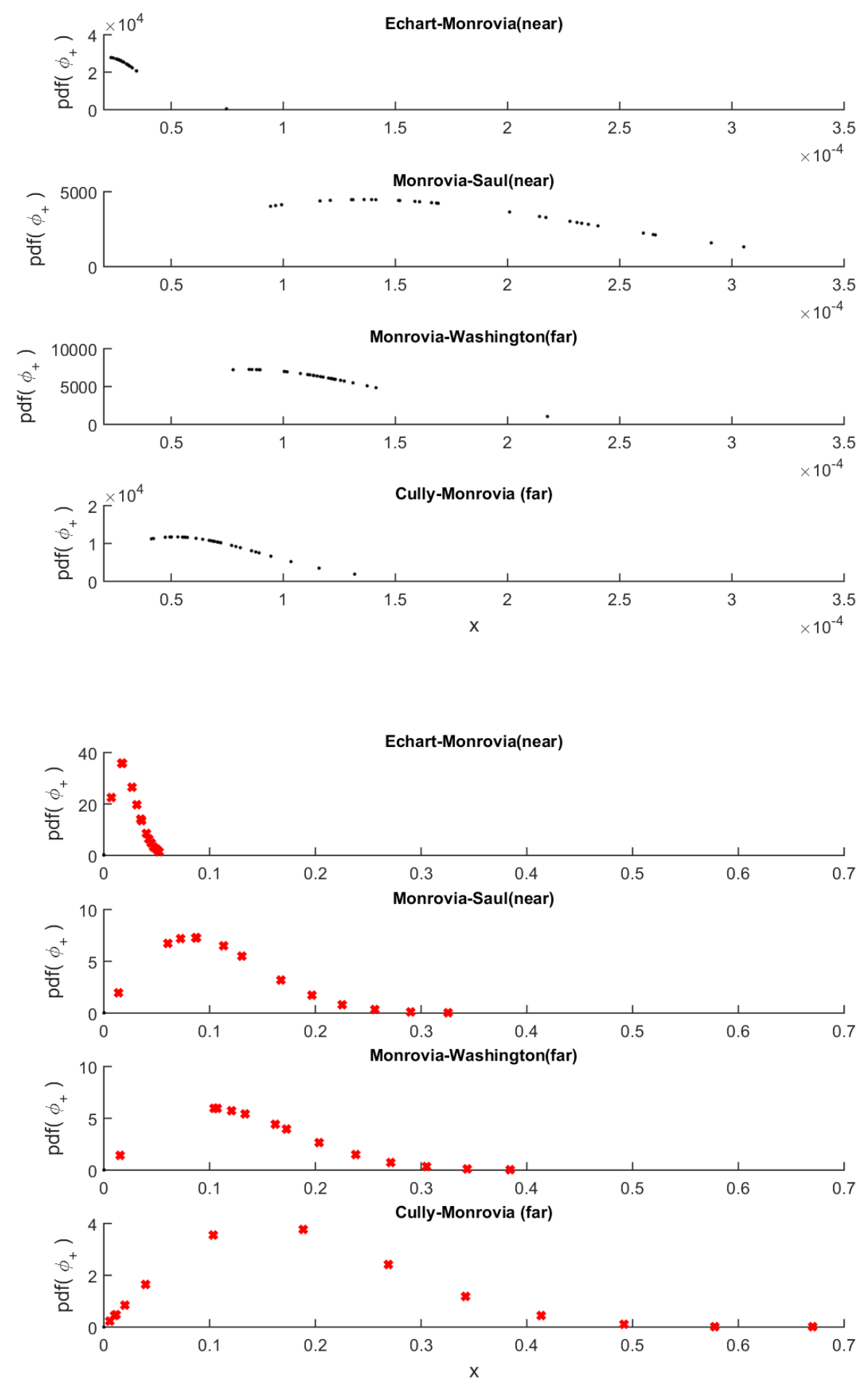

Figure 5.9: Rayleigh distribution of $\phi_{+}$over 30 cycle window size with $\mathrm{x}$-axis held constant for comparative purposes. (Top) Steady-state profile of Rayleigh prior to lightning strike. (Bottom) 13 cycles into a lightning event causing $x$ to deviate from steady-state. Note the decrease of the $\mathrm{x}$-axis scale compared to the previous figure. 

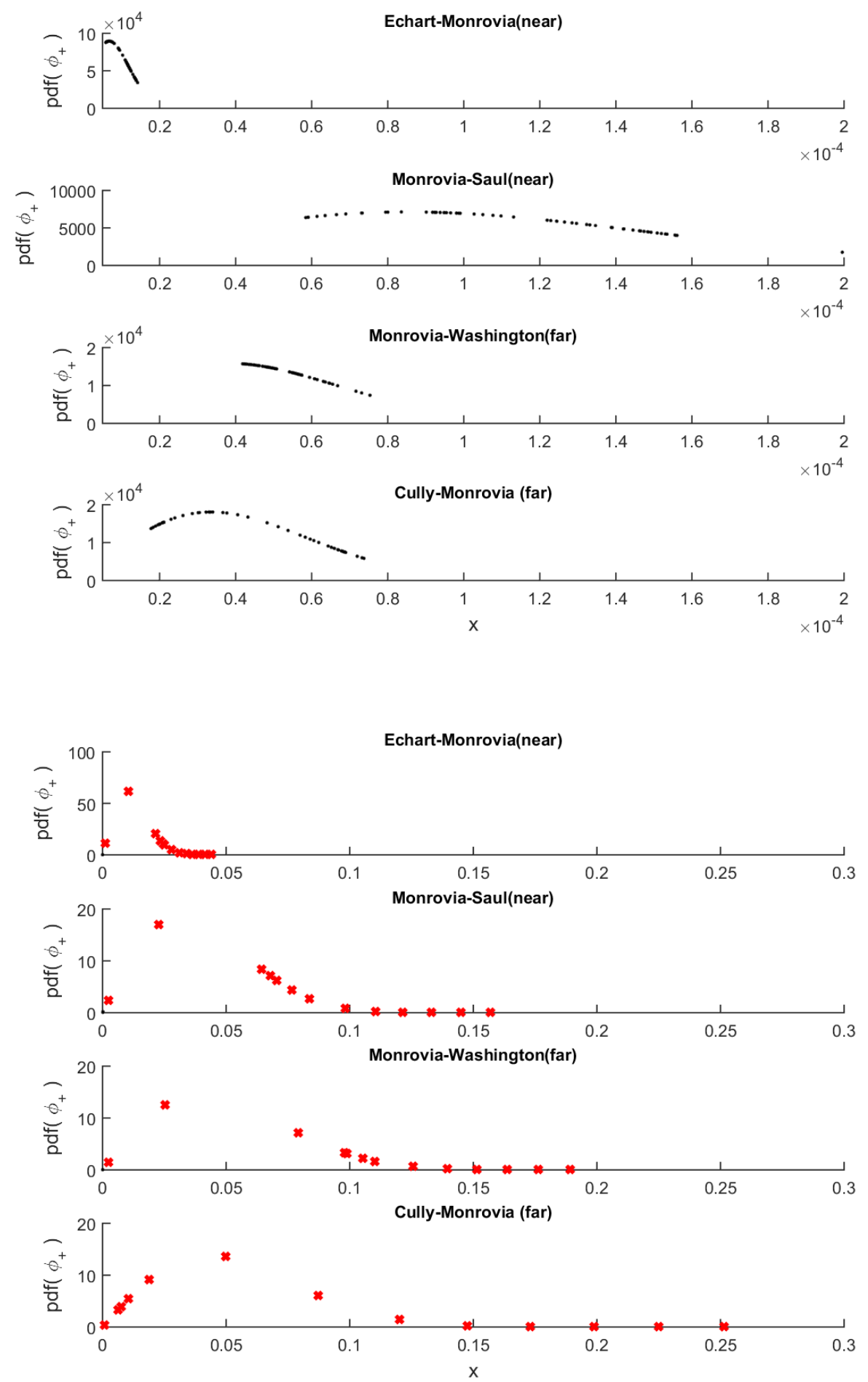

Figure 5.10: Rayleigh distribution of $\phi_{+}$over 60 cycle window size with $\mathrm{x}$-axis held constant for comparative purposes. (Top) Steady-state profile of Rayleigh prior to lightning strike. (Bottom) 13 cycles cycle into a lightning event causing $x$ to deviate from steady-state. Further, note the decrease of the $\mathrm{x}$-axis scale from the above two window sizes. 
To quantify the change in the spread of $x$ from steady-state to the event, when observing each PMU pair for each individual window size, $\sigma$ can be fitted to the Rayleigh distribution during these two instances. First, $\sigma$ is determined during steady-state (denoted $\sigma_{s s}$ ) and 13 cycles into the event $\left(\sigma_{\text {event }}\right)$. Next, the $\frac{\sigma_{\text {event }}}{\sigma_{s s}}$ ratio is determined for all clustered PMU pairs over 15,30 , and 60 cycle window size scenarios. Table 5.1 shows the $\frac{\sigma_{\text {event }}}{\sigma_{s s}}$ ratio for all clustered PMU pairs over 15, 30, and 60 cycle window size scenarios.

Table 5.1: Quantifying the change in the spread of $x$ of the Rayleigh distribution, during the lightning strike event compared to steady-state. Taking the ratio of $\sigma 13$ cycles into an event over $\sigma$ during steady state quantifies the change in the spread of $x$. Based on PMU pair, this ratio trends differently while increasing window size; Echart increases, whereas the rest vary. These, however, possess a smaller $\frac{\sigma_{\text {event }}}{\sigma_{s s}}$ ratio when going from a 15 cycle window size to a 60 cycle window size.

\begin{tabular}{|c|c|c|c|c|}
\hline 15 cycles & Echart & Saul & Washington & Cully \\
\hline & $2.20 \mathrm{E}+02$ & $5.20 \mathrm{E}+02$ & $3.80 \mathrm{E}+03$ & $2.00 \mathrm{E}+03$ \\
\hline 30 cycles & & & & \\
\hline & $7.80 \mathrm{E}+02$ & $6.10 \mathrm{E}+02$ & $4.20 \mathrm{E}+03$ & $2.90 \mathrm{E}+03$ \\
\hline 60 cycles & & & & \\
\hline & $1.40 \mathrm{E}+03$ & $3.80 \mathrm{E}+02$ & $1.10 \mathrm{E}+03$ & $1.30 \mathrm{E}+03$ \\
\hline
\end{tabular}

From Table 5.1, based on the observed PMU pair, $\frac{\sigma_{\text {event }}}{\sigma_{s s}}$ trends differently when increasing window size. For instance, based on Echart, $\frac{\sigma_{\text {event }}}{\sigma_{s s}}$ will increase with larger window sizes, whereas Saul, Washington, and Cully will vary. These sites, however, will remain consistent in that they possess a smaller $\frac{\sigma_{\text {event }}}{\sigma_{s s}}$ ratio when going from a 15 cycle window size to a 60 cycle window size. 


\subsection{Cluster Scheme}

To achieve maximum system monitoring, one might hypothesize that all PMU combinations within a balancing area must be correlated. This is, however, not necessary. To optimize event detection capabilities while preventing unnecessary and/or redundant computation, the algorithm need only monitor a subset of PMUs, so long as that cluster contains including both electrically-near and far PMUs. A near-far cluster of PMUs offers advantages over all-near or all-far clustering schemes. Recall from Chapter 4.9, using an all-near clustering scheme provides no reference to what constitutes an event; similarly for an all-far clustering scheme. By clustering a combination of both near and far PMUs and observing at the correlation layer, an event occurrence can be detected via far sites since correlation becomes decoupled with electrical distance. Furthermore, the event can be confirmed via near sites since adjacent sites experience events in a similar fashion and so their $\phi_{+}$will trend similarly.

In order show viability of this clustering scheme, the case study cluster will be concurrently analyzed with one small and one large window size. Figure 5.11 plots the normalized Rayleigh distribution variable, $x / \mu(x)$, prior to a lightning strike event, as shown with blue '? markers. Each PMU pair has its own predetermined threshold value, shown within the title portion of the PMU pair in parenthesis. Recall from Chapter 4.5.1, these threshold values were established to be the lowest allowable value which does not classify false-positives while preserving event detection. 

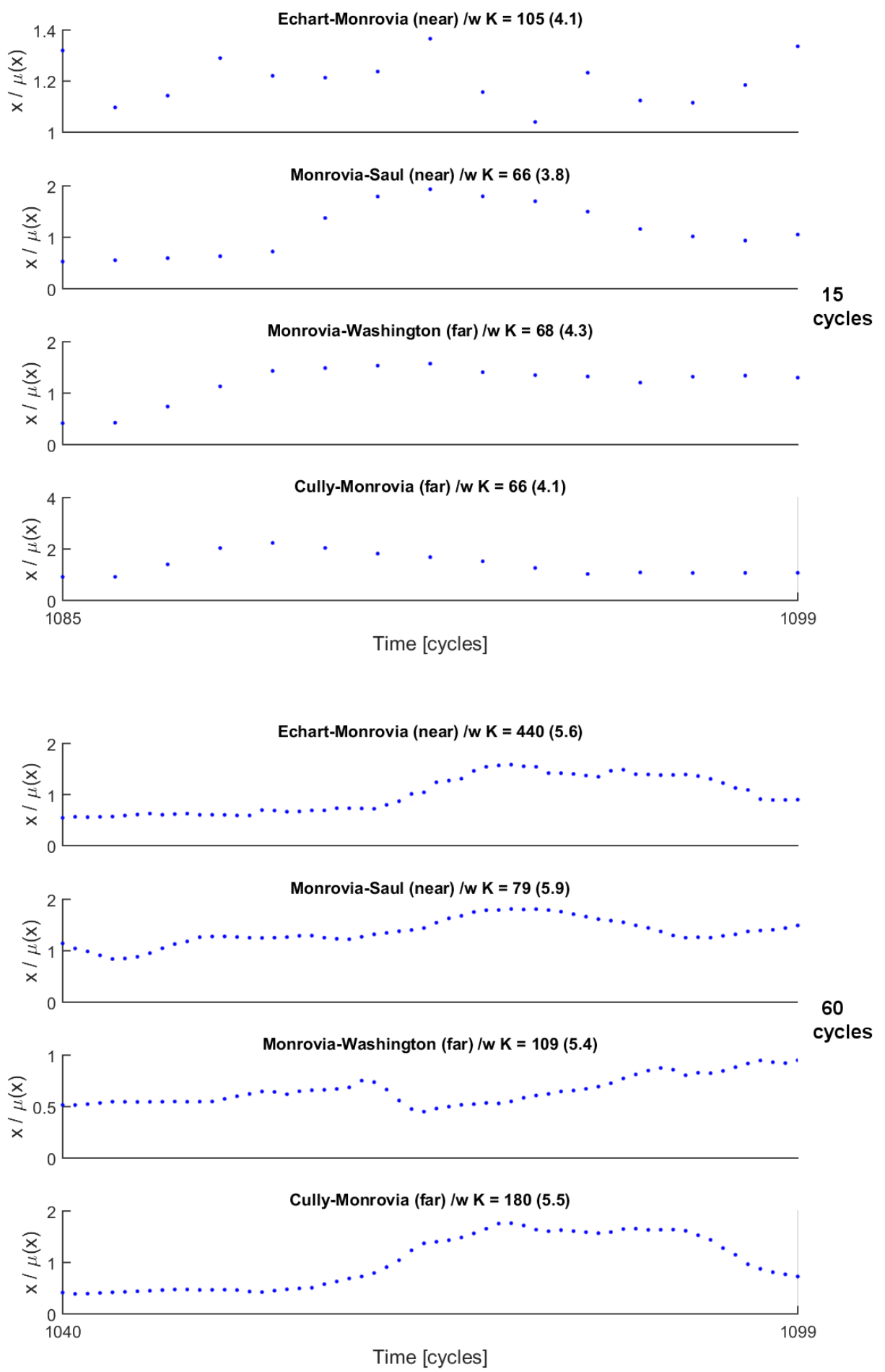

Figure 5.11: Normalized $x$ values, during steady-state, for the case study cluster, prior to a lightning strike occurrence, over a 15 and 60 cycle window size. Threshold values for each individual PMU pair are shown along the title portion in parenthesis. 
Observing 13 cycles into the event, Figure 5.12 imposes these threshold values as a solid black horizontal bar and shows event-related data that does not exceed the threshold value as red '.' markers. Normalized $x$ values that exceed the threshold are shown as red ' $\mathrm{x}$ ' markers.

From Figure 5.12, observing the 15 cycle window size scenario, normalized $x$ values will begin to exceed the threshold value a few cycles into the event. About five cycles in, however, a majority of the normalized $x$ values go below the threshold value. Some sites, namely Cully, will experience normalized $x$ values oscillate about the threshold value repeatedly. Declaring an event based solely on this window size would be inadequate given the limited amount of time to declare the event and the uncertainty caused by the oscillation at Cully.

Now, observing the 60 cycle window size, normalized $x$ values exceed the threshold within a few cycles into the event as well. Moreover, nearly all normalized $x$ values continue to exceed the threshold value 13 cycles into the event for all PMUs. To ensure no oscillations occur, as with Cully in the 15 cycle window size scenario, Figure 5.13 observes the 60 cycle window size scenario 40 cycles into the event. It can be seen that normalized $x$ values gradually decrease towards its steady-state profile. Therefore, a large window size can be used with certainty to detect the event occurrence while a small window size can confirm that an actual power system event. This demonstrates that concurrent analysis with two window sizes over this clustering scheme is viable for detecting these types of events in this area of the synchrophasor network. 

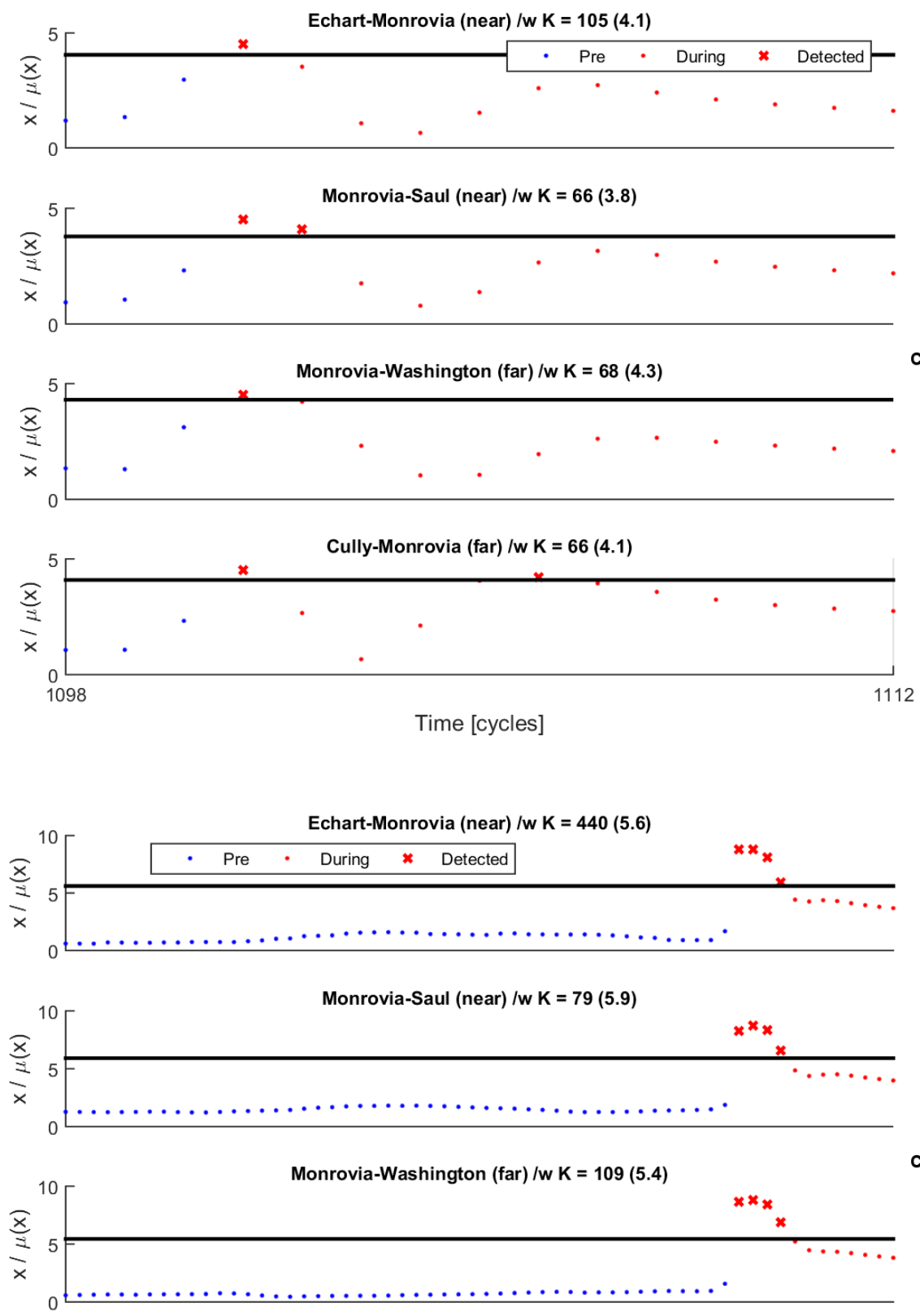

cycles

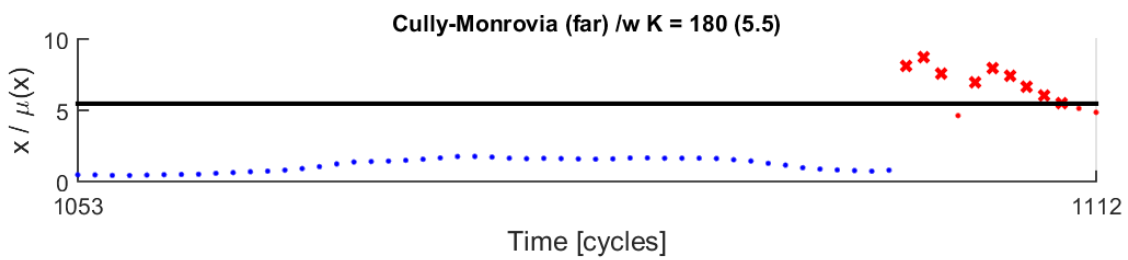

Figure 5.12: Normalized $x$ values, 13 cycles into the lightning strike occurrence, for the case study cluster over a 15 and 60 cycle window size. Note that, when observing with the 60 cycle window size, normalized $x$ values exceed the threshold in a few cycles. Furthermore, nearly all of the normalized $x$ values continue to exceed the threshold value well into the event among all clutered PMUs. 

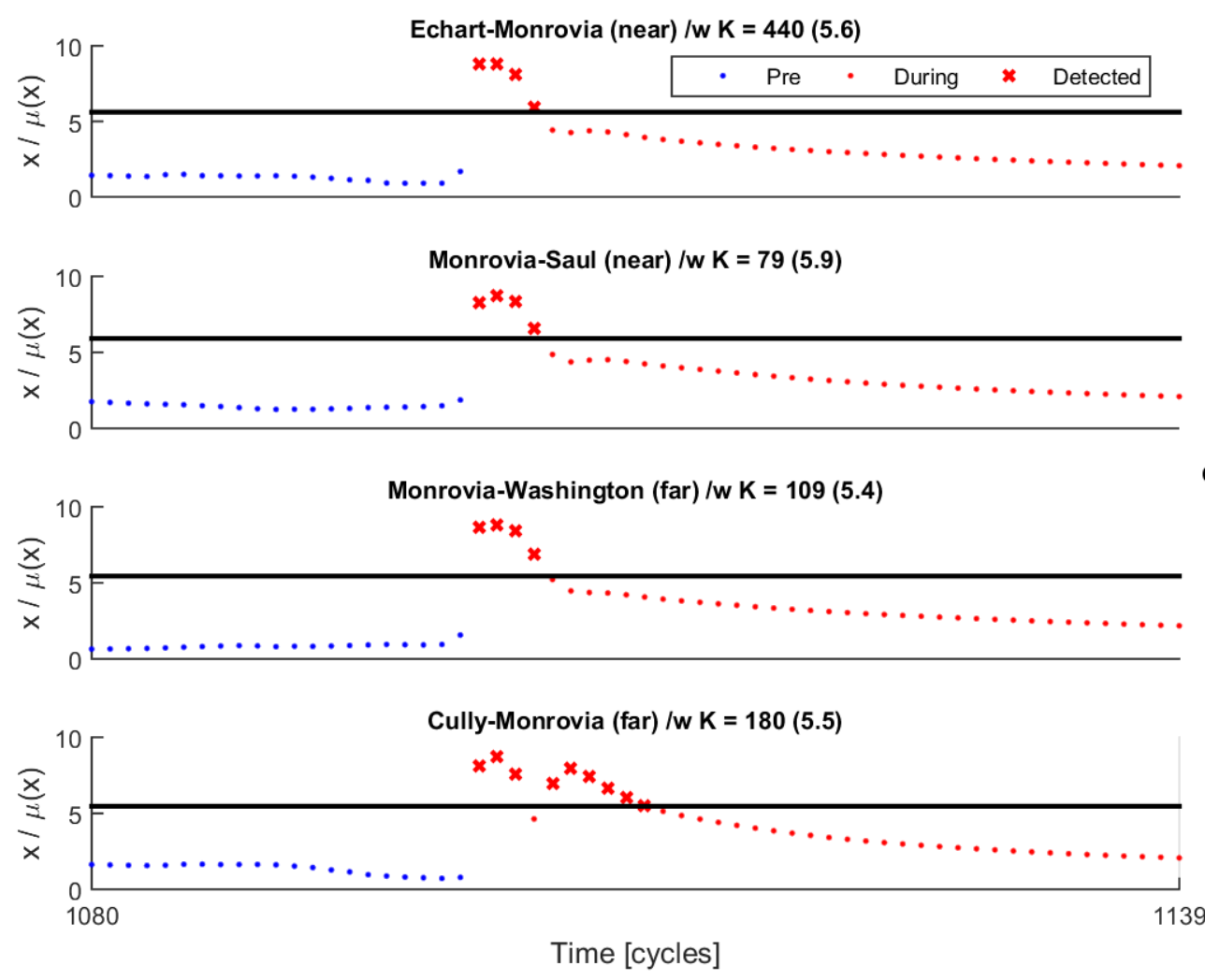

Figure 5.13: Normalized $x$ values, 40 cycles into the lightning strike occurrence, for the case study cluster over a 60 cycle window size. This is done to ensure no oscillations of normalized $x$ occur about the threshold as shown in Figure 5.12 with the 15 cycle window size. 


\subsubsection{Analyzing Other Events}

In order to verify this clustering scheme provides adequate detection, the remaining events were analyzed in a similar fashion to the above. Unfortunately, all of these events occur at or near the same location. Regardless of this limitation, Figure 5.14 shows the time domain response for site Monrovia and Cully during another lightning strike event, dubbed event 2 . Figure 5.15 shows the time domain response over the case study cluster. Figure 5.16 depicts the visual structure 13 cycles into the event over the entire system. Figures 5.17 and 5.18 show the normalized $x$ prior to and 13 cycles into the event, over a 15 and 60 cycle window size. Analysis occurs over the same case study cluster but differs from the previous event in that the established threshold values are unique to this event. 

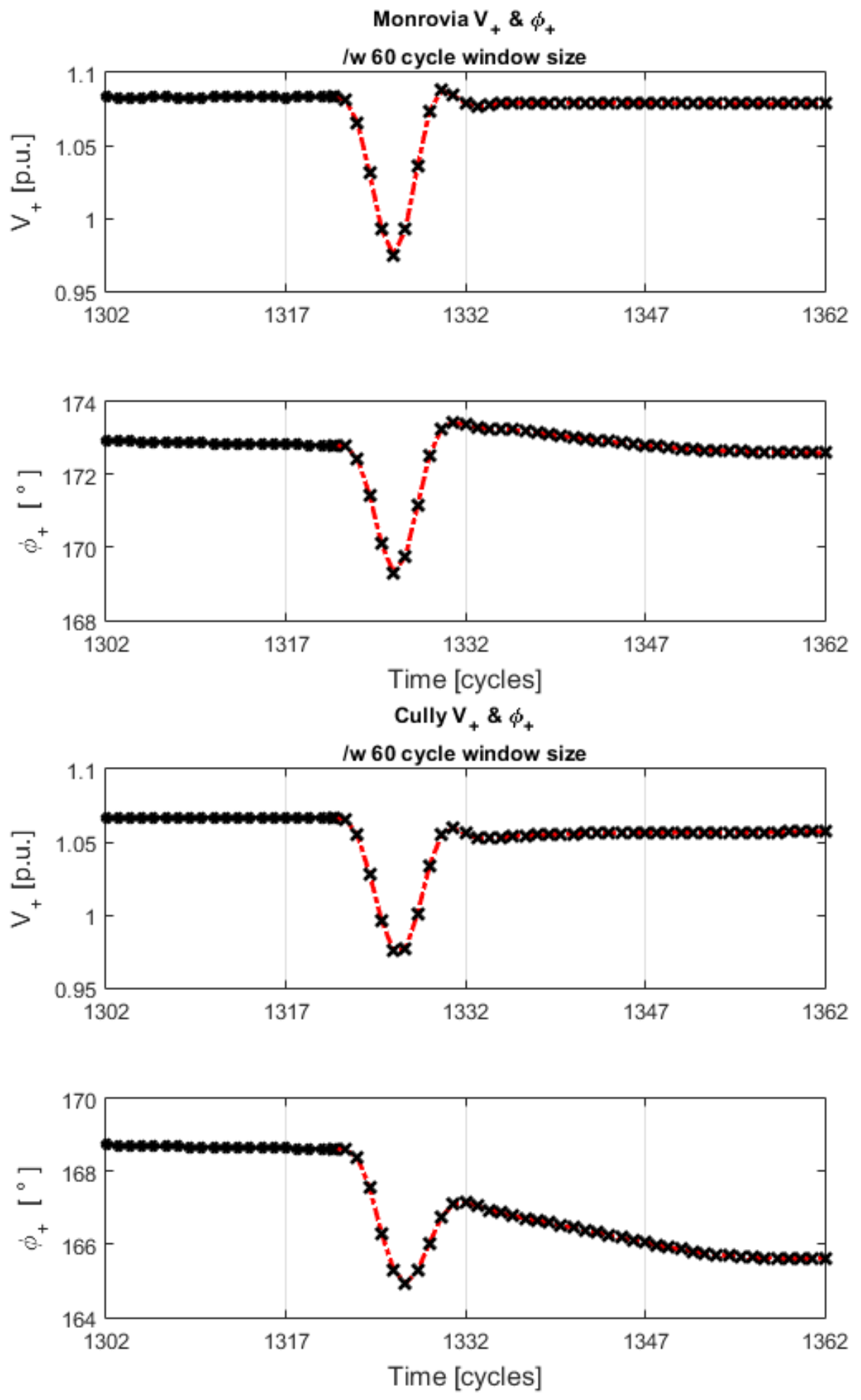

Figure 5.14: Time domain response of $V_{+}$and $\phi_{+}$, for Monrovia and Cully, 40 cycles into event 2 over a 60 cycle window size. 

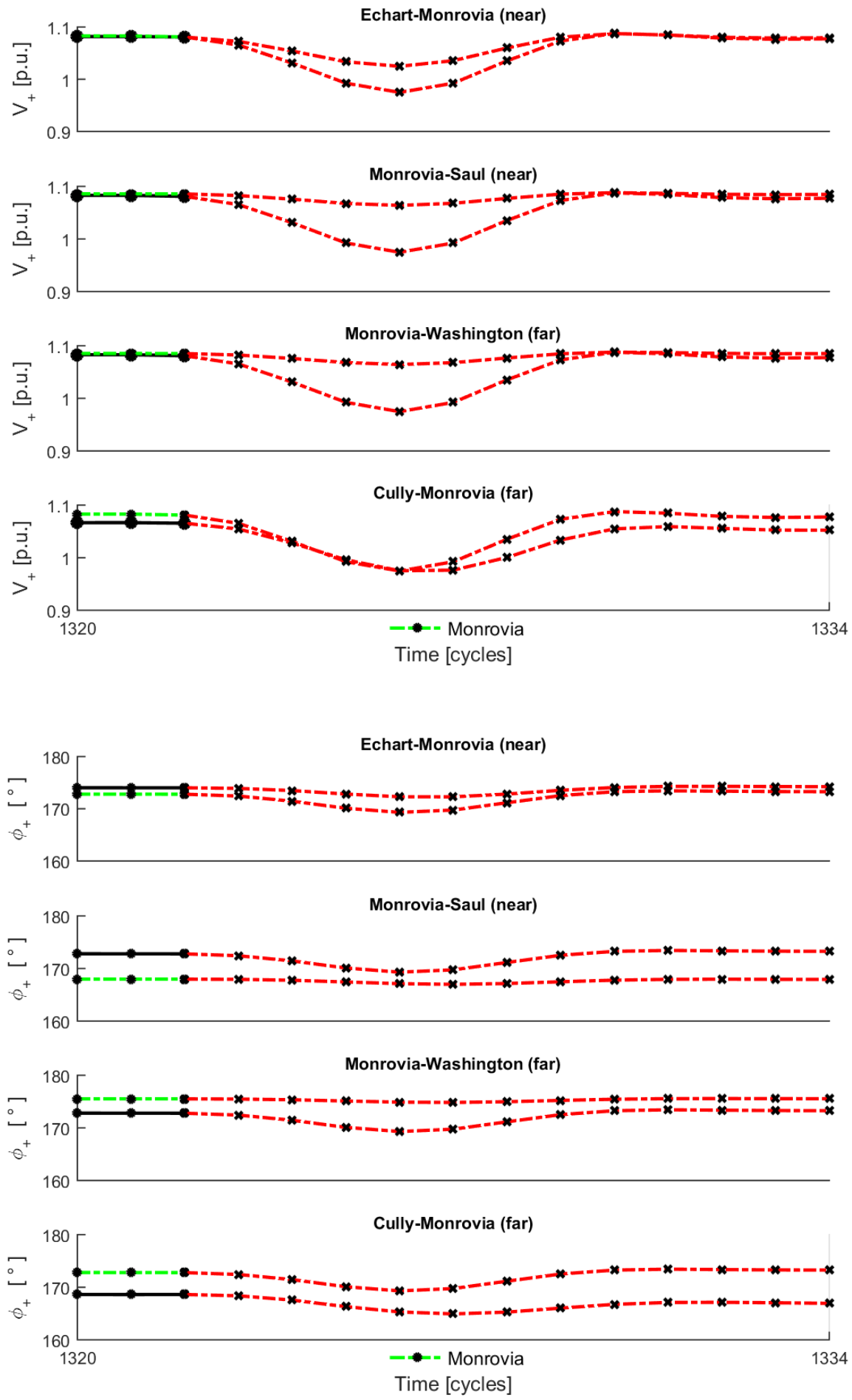

Figure 5.15: Time domain plot of $V_{+}$and $\phi_{+}, 13$ cycles into event 2 over a 15 cycle window size, as captured by case study cluster. 


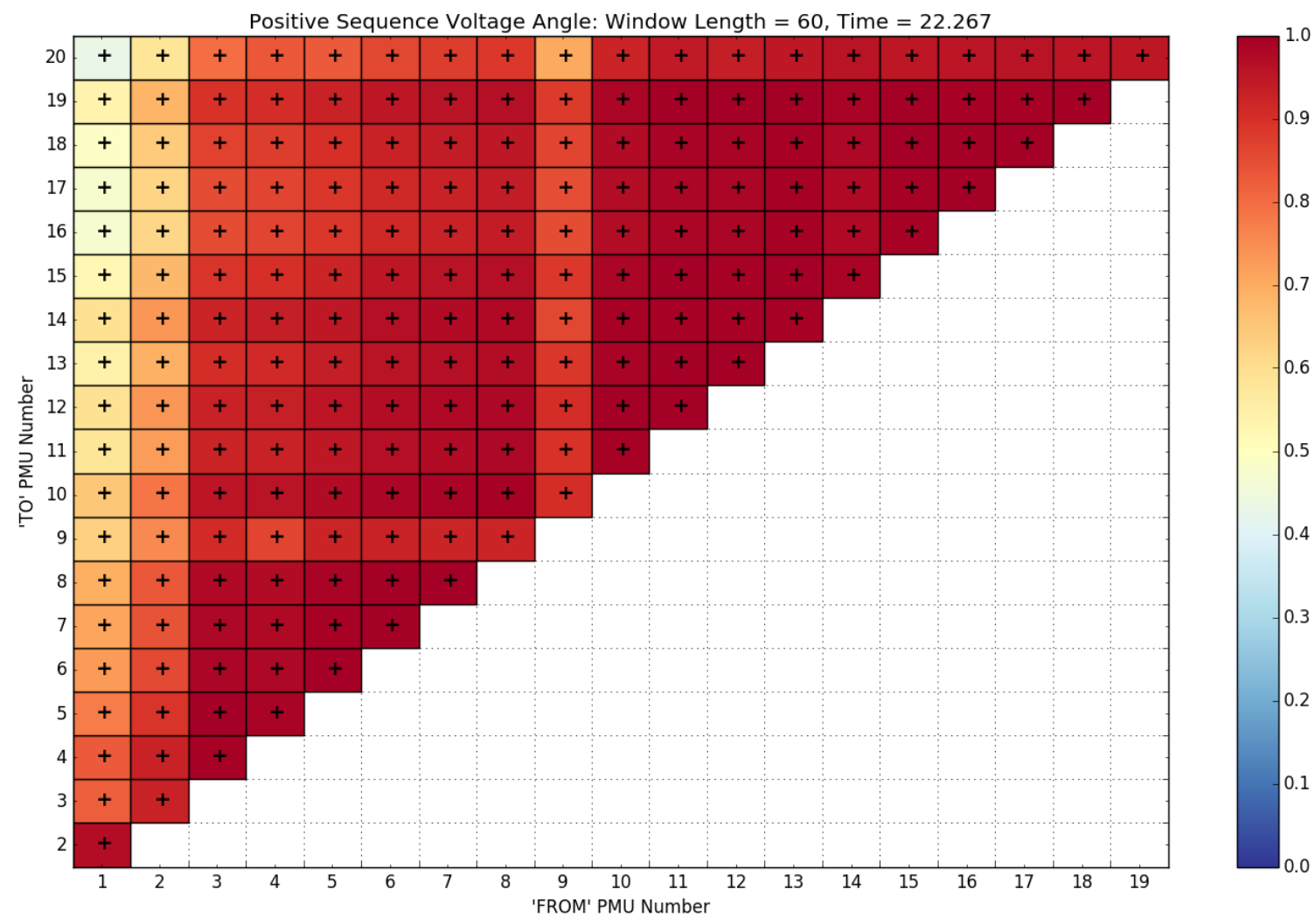

Figure 5.16: Visual structure 13 cycles into event 2 over the entire system. Correlation with the sites experiencing an event most strongly (in this instance PMU Number 1 - Monrovia, PMU Number 2 - Echart, and to a less degree PMU Number 9 - Cully) have become decoupled, indicating an event has occurred between these sites (most likely between Monrovia and Cully since Echart is so electrically close to Monrovia). 

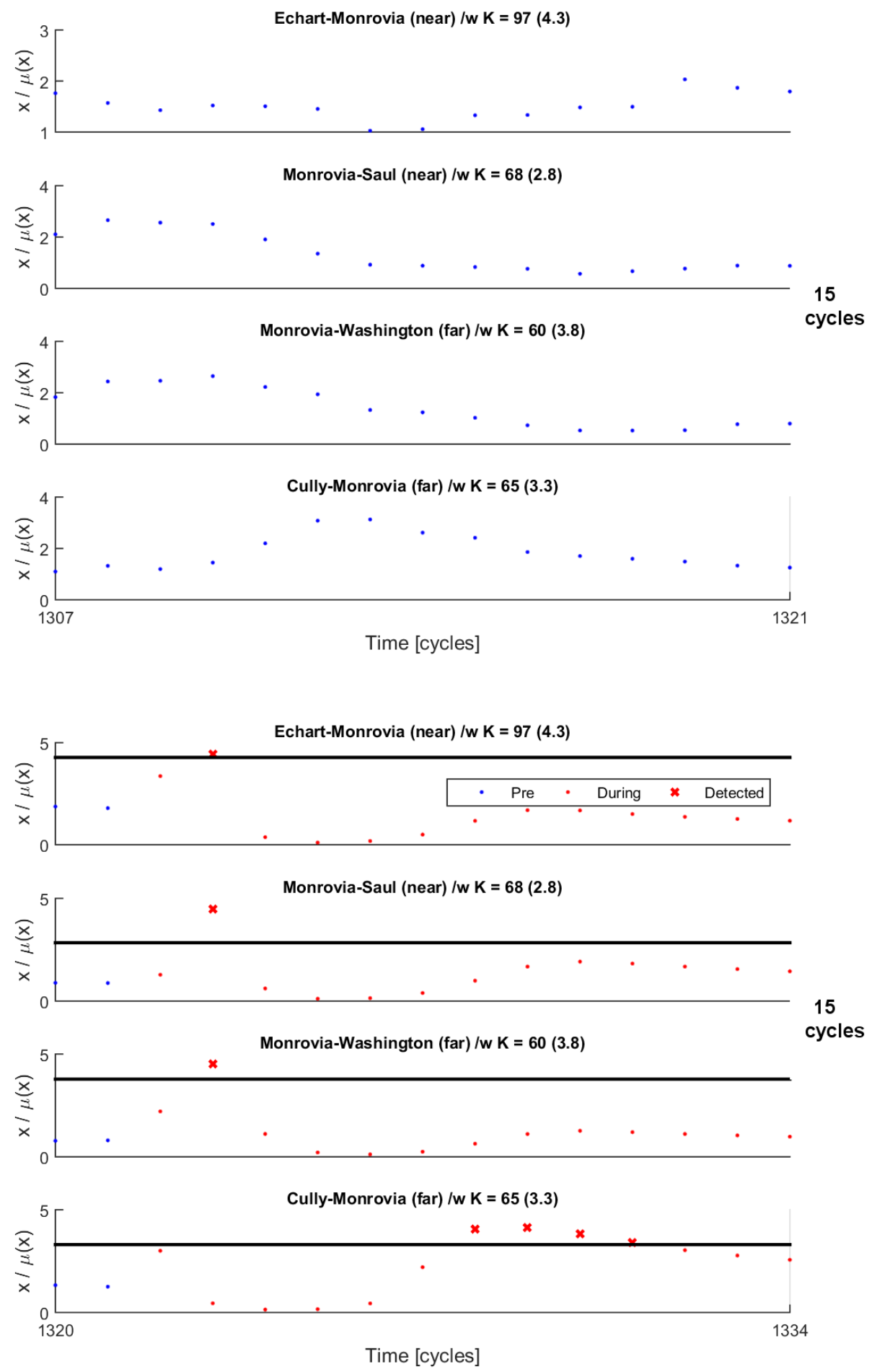

Figure 5.17: Normalized $x$ values during steady-state and 13 cycles into event 2, for the case study cluster over a 15 cycle window size. Threshold values, at this particular window size and event, for each individual PMU pair, are shown along the title portion in parenthesis. 

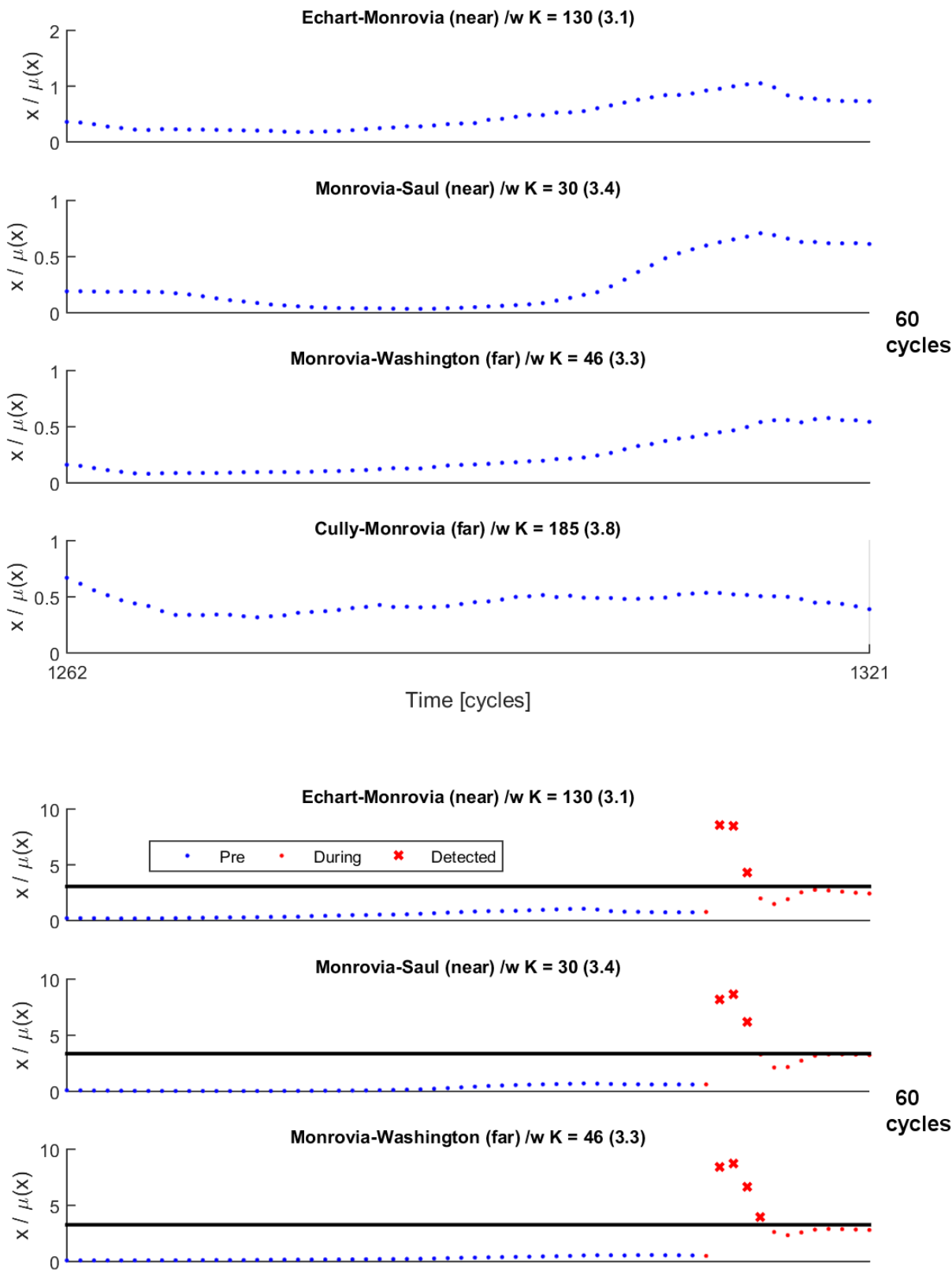

cycles

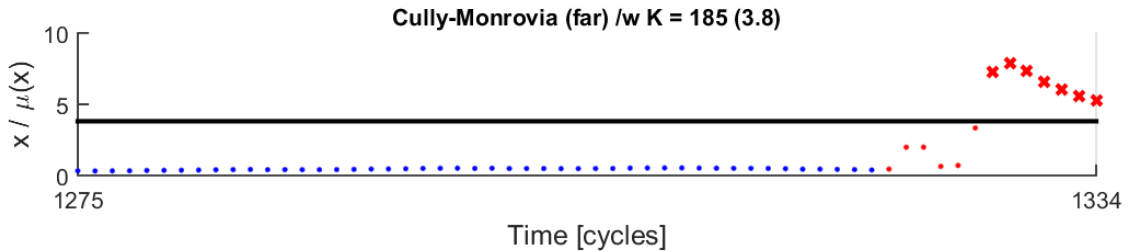

Figure 5.18: Normalized $x$ values during steady-state and 13 cycles into event 2, for the case study cluster over a 60 cycle window size. Threshold values for each individual PMU pair, at this particular window size and event, are shown along the title portion in parenthesis. 
As before, we observe 40 cycles into the event, over a 60 cycle window size, to ensure no oscillations occurs about the threshold value. Figure 5.19 provides the visual structure during this time. From Figure 5.20, normalized $x$ values exceeded the threshold over the entire cluster for an acceptable amount of cycles, but not all at the same time. When the threshold is exceeded for Cully, the threshold is no longer exceeded for remaining PMUs.

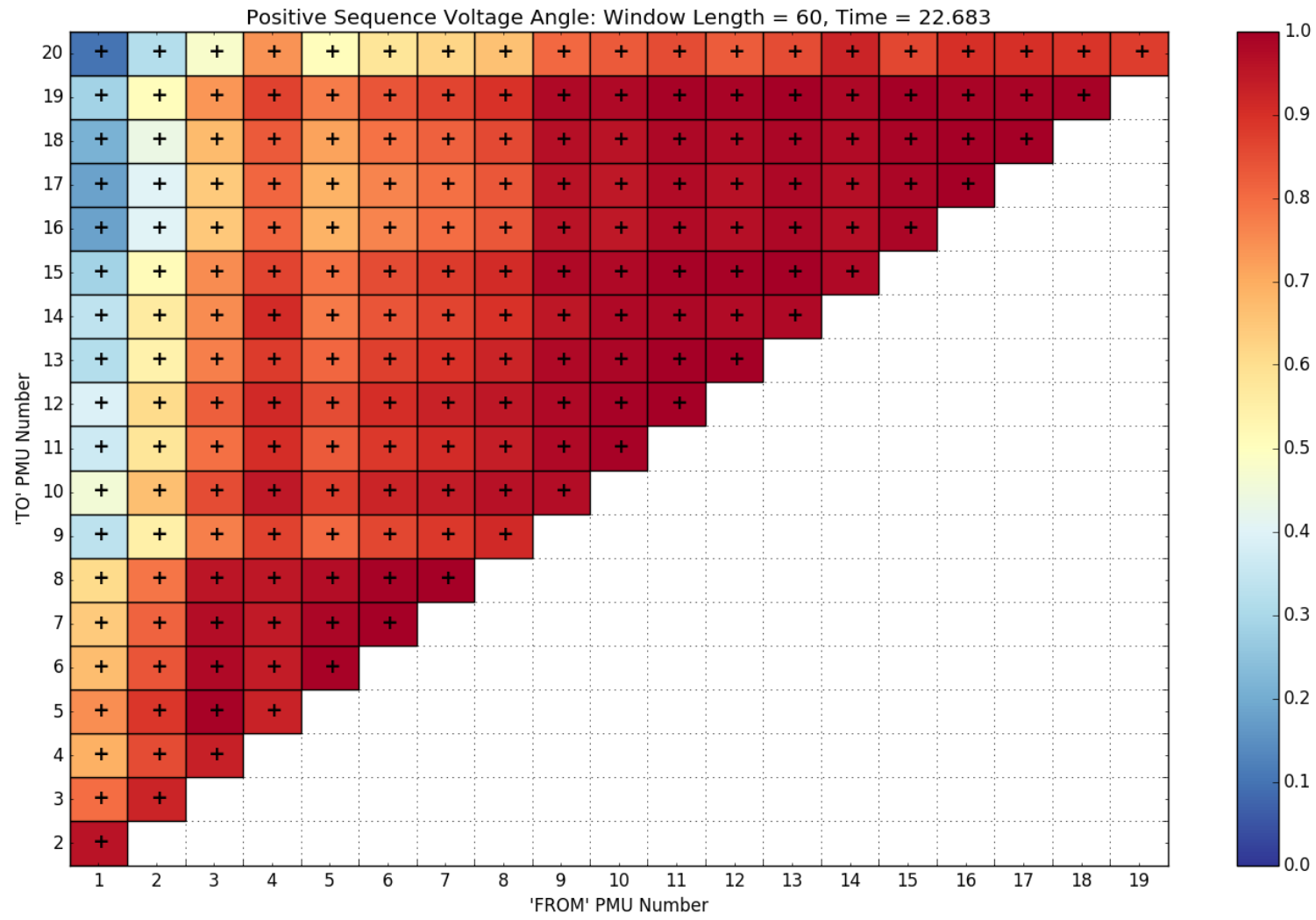

Figure 5.19: Visual structure 40 cycles into event 2 - Monrovia becomes further decorrelated with the system compared to Figure 5.16. 

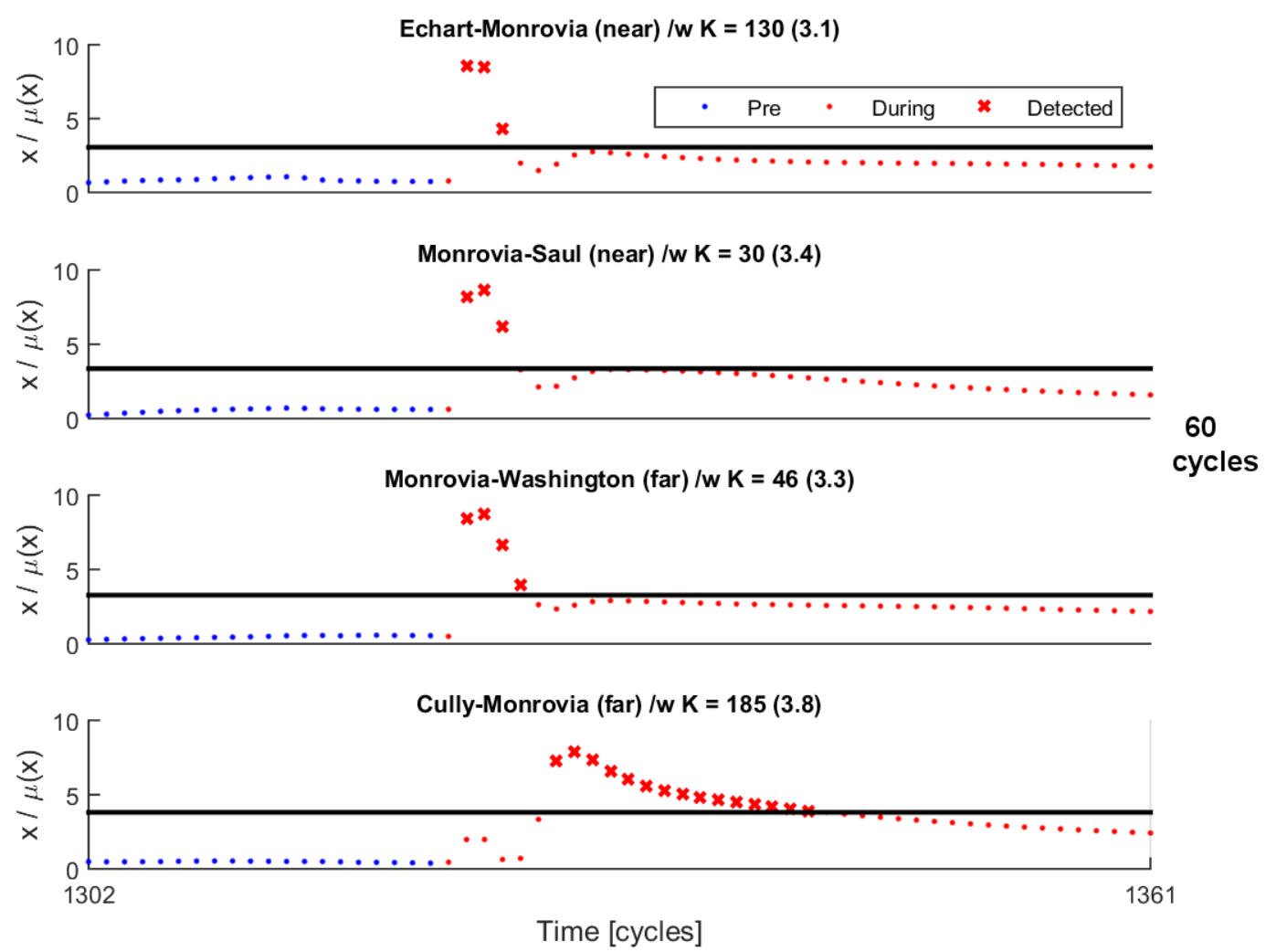

Figure 5.20: Normalized $x$ values, 40 cycles into event 2, for the case study cluster over a 60 cycle window size. Observing 40 cycles into this particular event, no oscillations of normalized $x$ occur about the threshold as shown in Figure 5.17 at Cully over a 15 cycle window size.

To contend with this issue, a minimum amount of PMUs need to be clustered. This way, simple voting schemes can be developed in which a majority of the cluster can vote to declare an event. For instance, for an individual PMU in the cluster, a certain amount of cycles need to consecutively exceed the threshold before declaring an event occurrence. Because this may not happen at the same time, once an event has been declared, a flag is set high and held for a certain amount of cycles in order to wait for other clustered sites to declare an event. If a majority of the cluster declares an event during this period, higher-level applications can be informed of its occurrence. In addition, the majority of the cluster could 
be constrained to require, at minimum, one near and one far PMU to declare an event. This type of scheme would be simple to integrate and would minimally add to computation time. Implementing such as a scheme is advantageous since it would minimize insecure operations from taking place.

Let us observe yet another lightning strike incident around Monoriva, dubbed event 3. Figure 5.21 shows the time domain response for Monrovia 13 cycles into the event. Figure 5.22 shows the response over the entire cluster. Figure 5.23 depicts the visual structure prior to and 13 cycles into the event over the entire system. Figures 5.24, 5.25, and 5.26 provide the same analysis as the above two events. Again, the established threshold values are unique to this particular event. A notable difference here is the Washington site being offline during this period and so was excluded from the cluster. From Figure 5.26, as with the previous analysis, since normalized $x$ values exceeded the threshold over the entire cluster for an acceptable amount of cycles, the event detection metric holds for this event as well. 

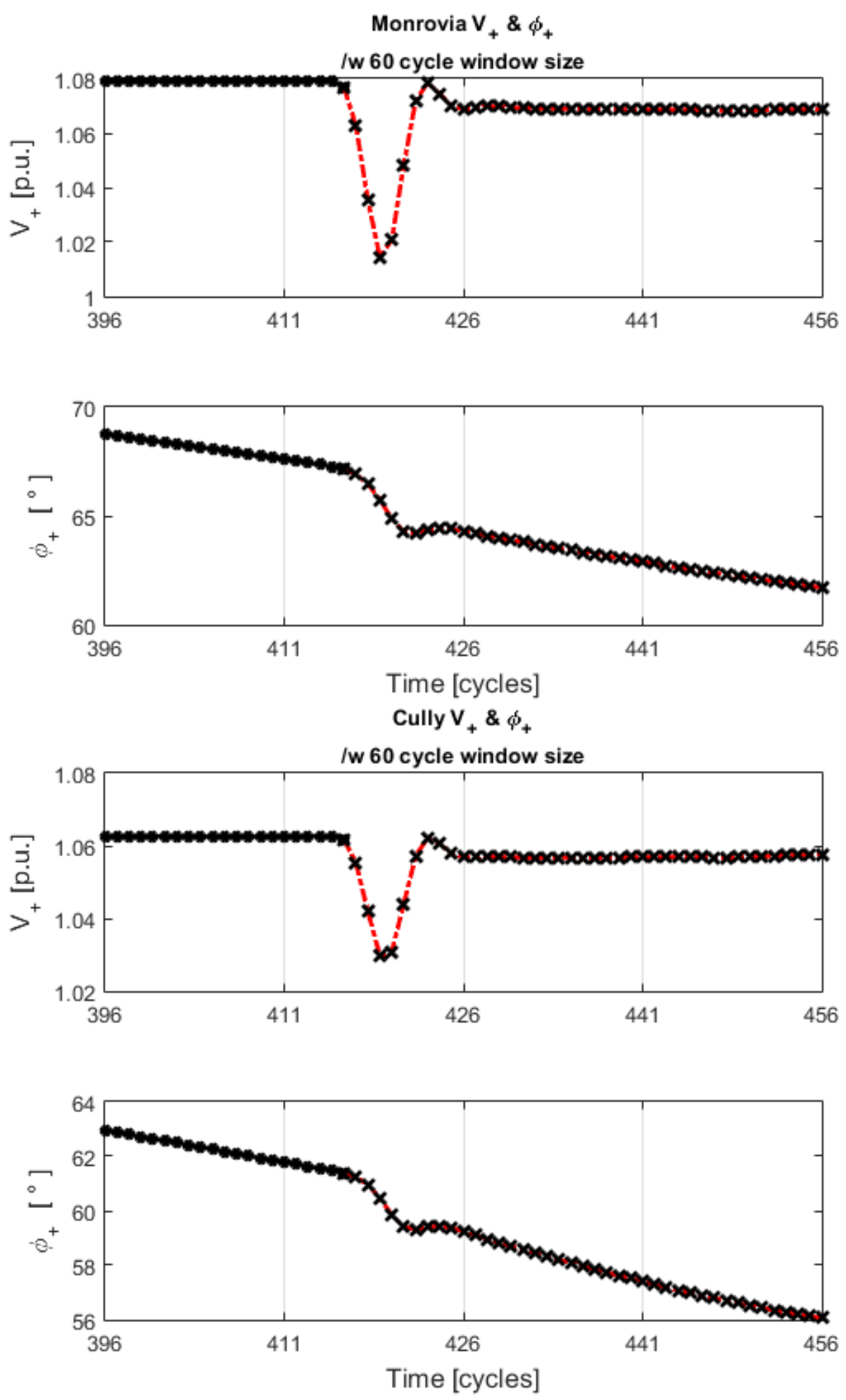

Figure 5.21: Time domain response of $V_{+}$and $\phi_{+}$for Monrovia and Cully, 40 cycles into event 3 over a 60 cycle window size. 

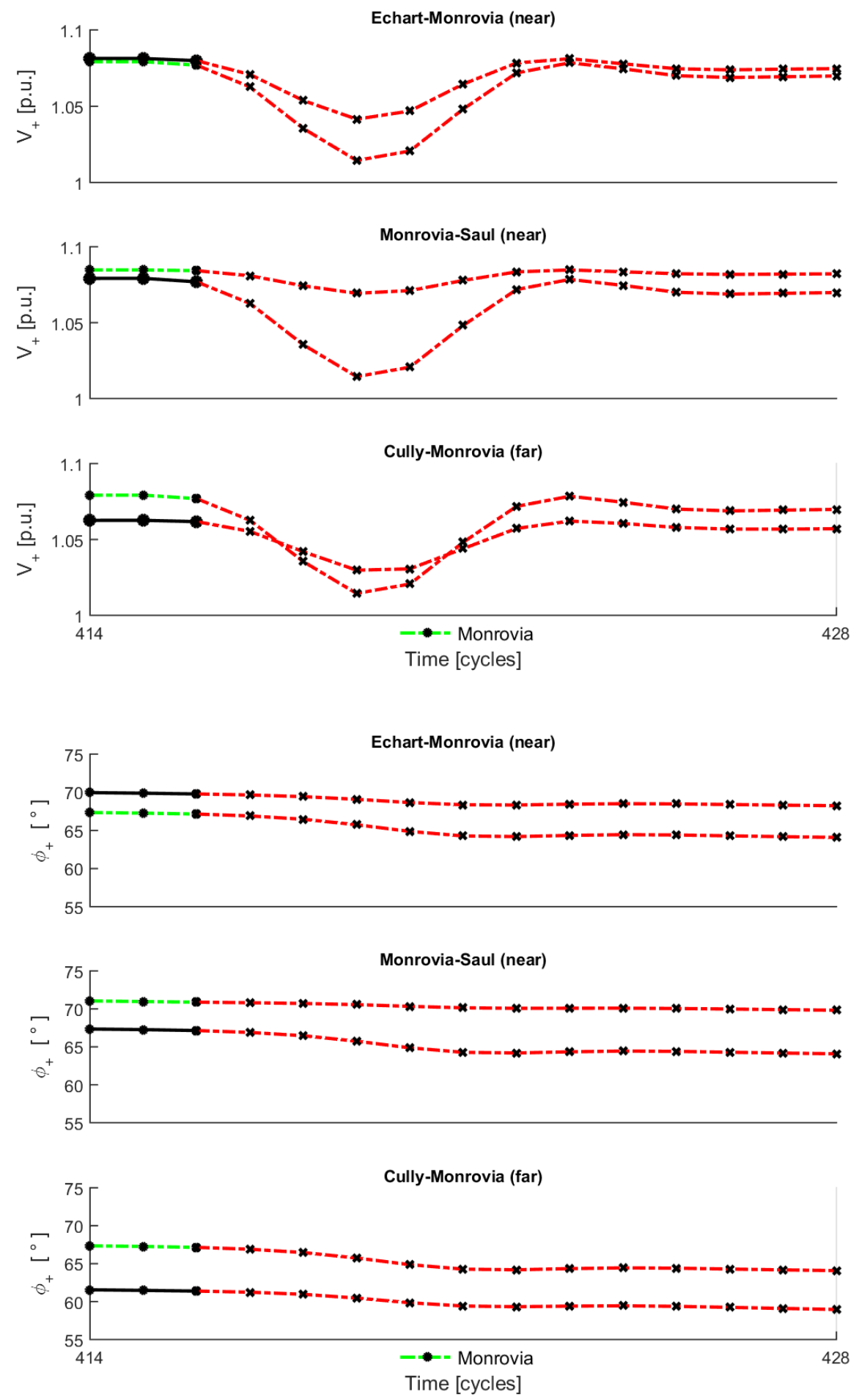

Figure 5.22: Time domain plot of $V_{+}$and $\phi_{+}, 13$ cycles into event 3 , as captured by case study cluster. 


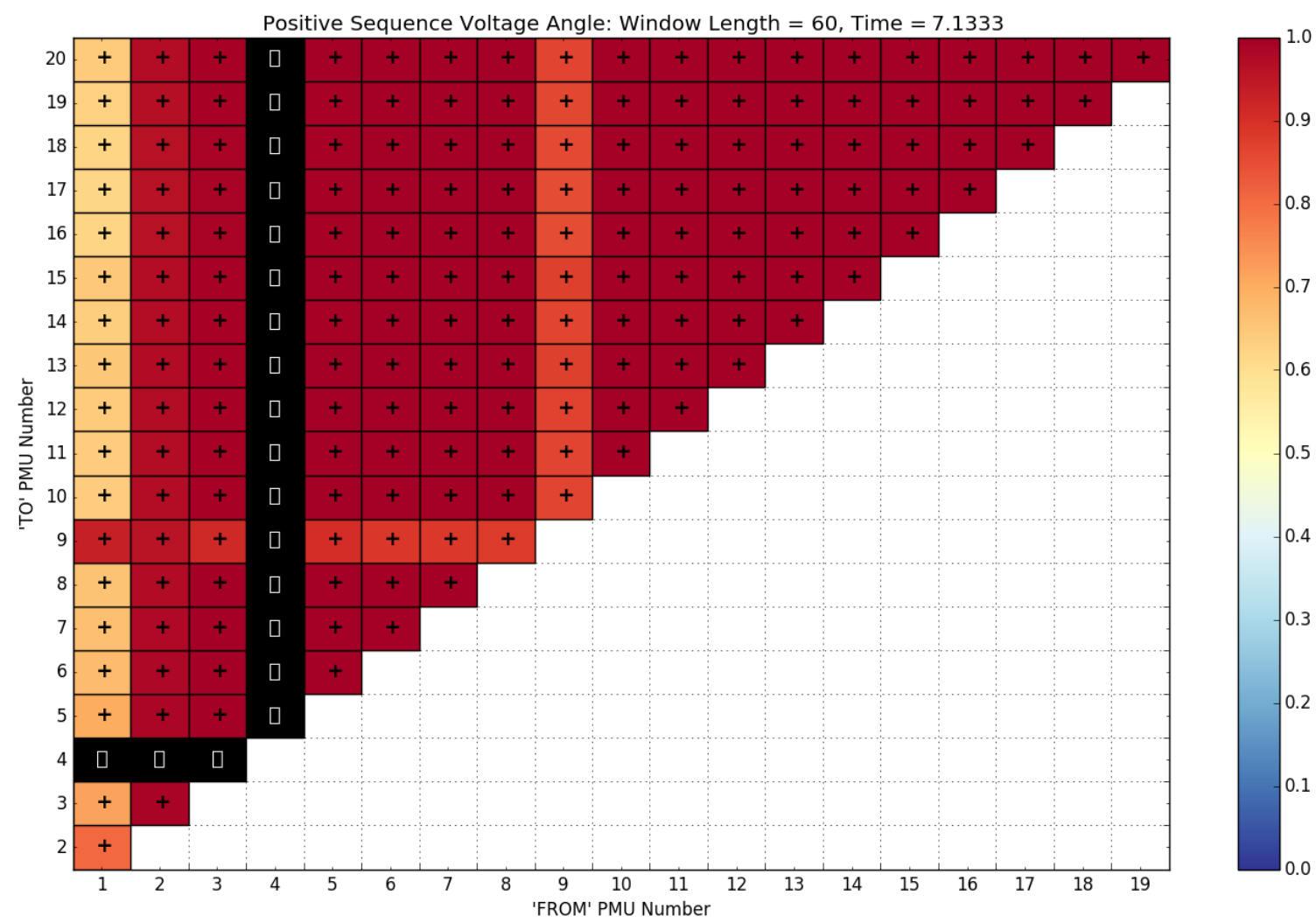

Figure 5.23: Visual structure 13 cycles into event 3 over the entire system. As with event 2, the lightning strike occurrence is between Monrovia and Cully. Because a (slight) gradient of decorrelation is displayed throughout site comparisons less than PMU number 9, and is more uniform throughout site comparisons greater than PMU Number 9, the event must have occurred closer to Cully. Note PMU Number 4 is the offline site, Washington. 

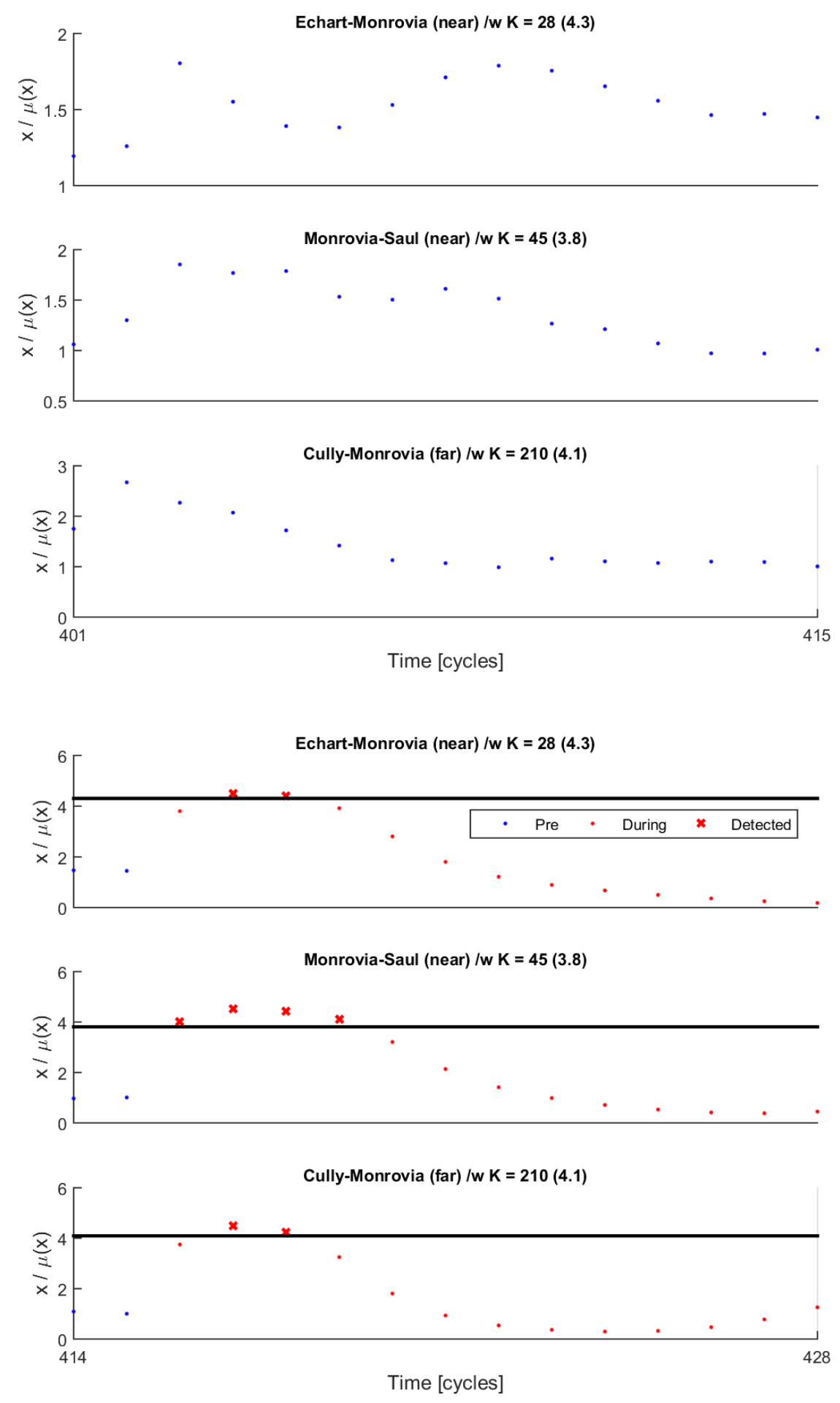

Figure 5.24: Normalized $x$ values during, steady-state and 13 cycles into event 3 , for case study cluster over a 15 cycle window size. Threshold values for each individual PMU pair, at this particular window size and event, are shown along the title portion in parenthesis. 

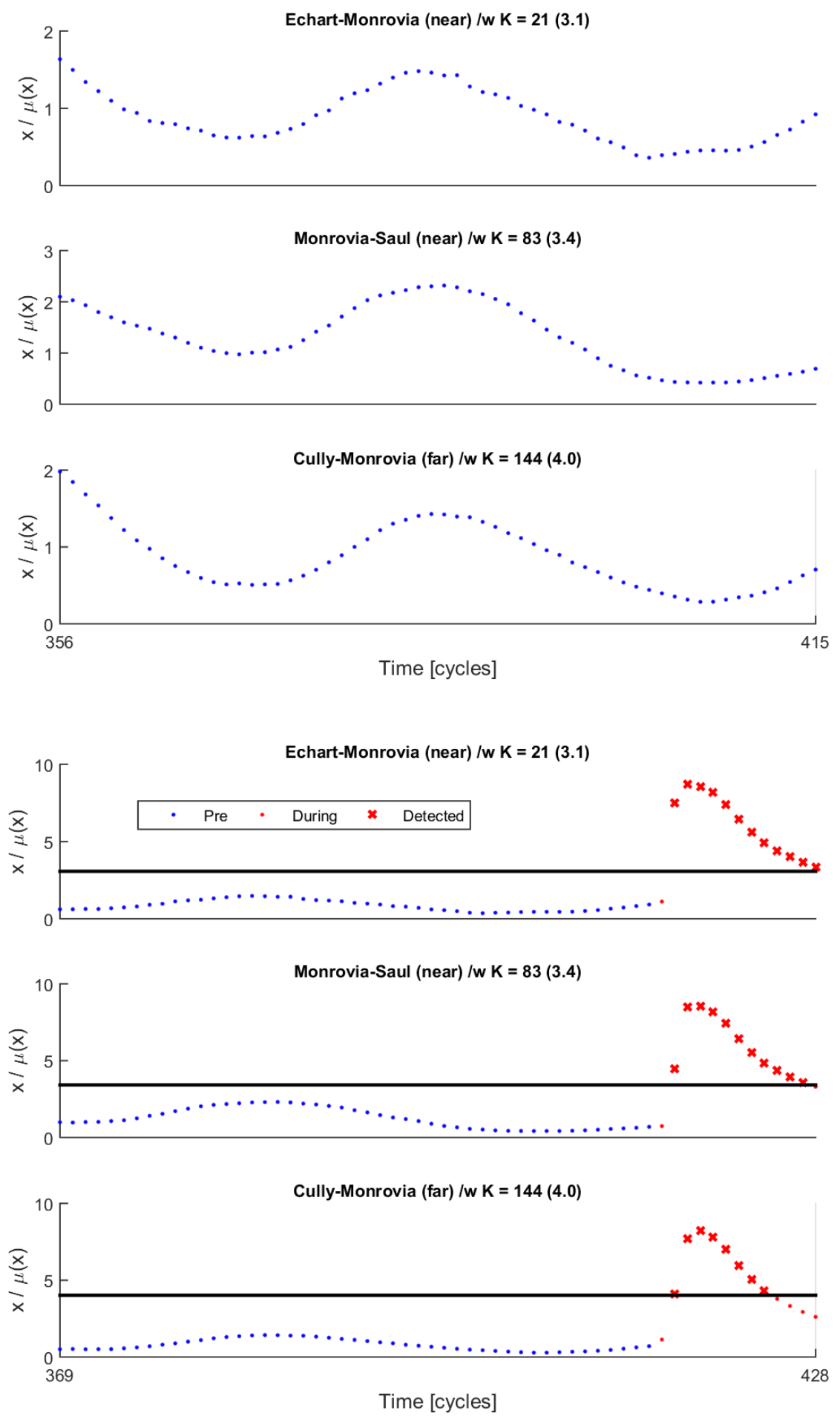

Figure 5.25: Normalized $x$ values during steady-state and 13 cycles into event 3 , for the case study cluster over a 60 cycle window size. Threshold values for each individual PMU pair, at this particular window size and event, are shown along the title portion in parenthesis. 

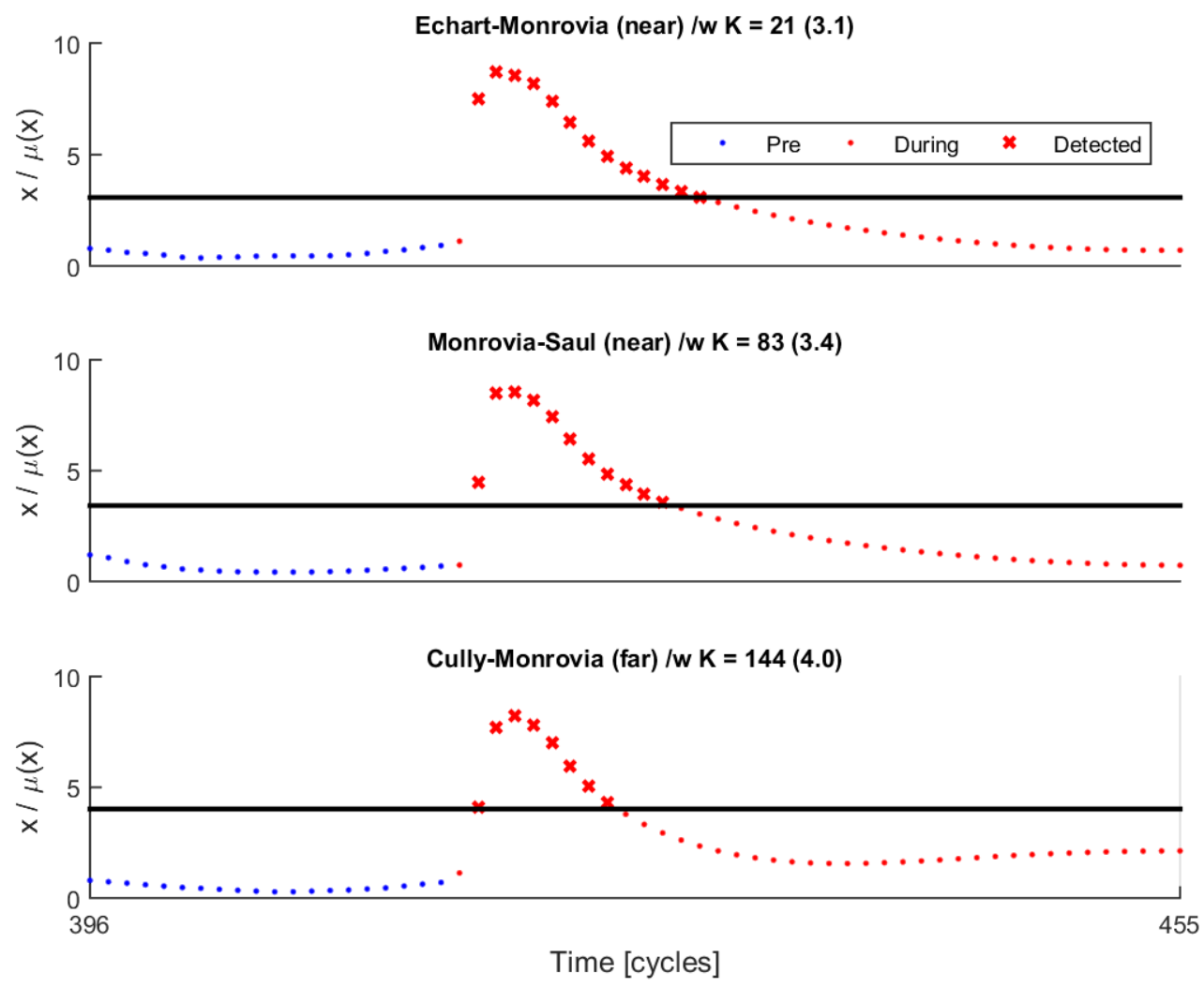

Figure 5.26: Normalized $x$ values, 40 cycles into event 3, for the case study cluster over a 60 cycle window size. Observing 40 cycles into this particular event, no oscillations of normalized $x$ occur about the threshold as shown with previous events. 


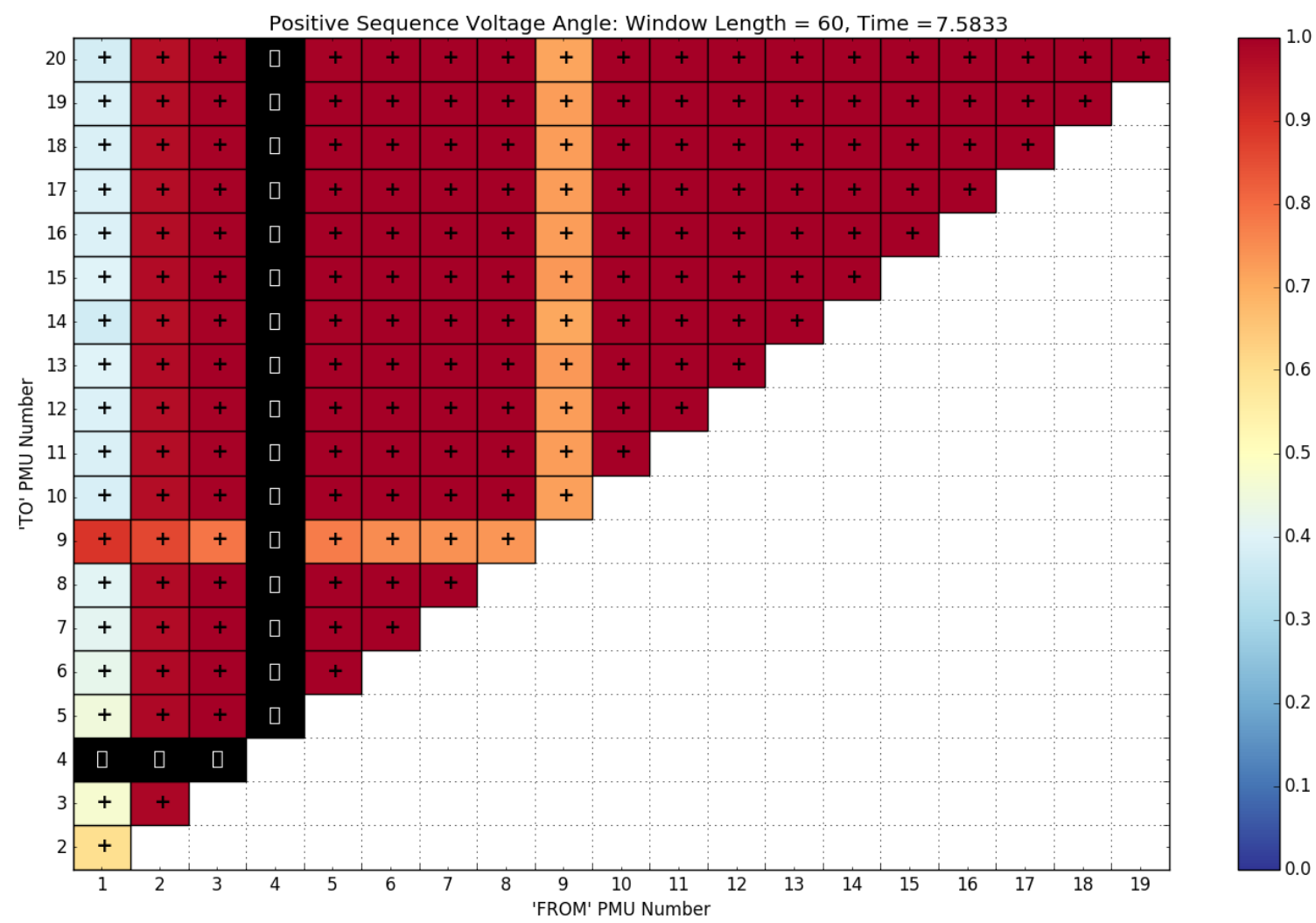

Figure 5.27: Visual structure 40 cycles into event 3. Monrovia and Cully become further decorrelated with the system compared to Figure 5.23. While correlation remains uniform throughout site comparisons greater than PMU Number 9, the gradient of decorrelation becomes more pronounced for site comparisons less than PMU Number 9. 


\section{Discussion}

To contend with data-related issues, Chapter 5.1 demonstrated the capability of Pearson correlation detecting both data drift and drop occurrences. Using the visual structure, we are able to qualitatively depict when these types of issues occur. Similarly, from the Pearson equation, we are able to quantify these types of data-related events.

To increase robustness in event detection, Chapter 5.2 demonstrated the benefits gained when varying the window size. Since the variance decreases as window size increases, we see much less variability with $x$, making our event detection metric much more sensitive to sudden changes in $\phi_{+}$when using large window sizes. Using the visual structure, again, we are able to qualitatively depict when lightning strikes occur within the system with a varying window size. To take advantage of this robustness, concurrent analysis over the same data stream should occur with, at least, a small and large window size.

Using parameters from the Rayleigh distribution, we are able to quantify these lightning strike occurrences. From Chapter 5.3, it has been demonstrated, both quantitatively and qualitatively, signatures of a Rayleigh PDF during these types of events occur. Furthermore, parameters from this distribution are used to detect these type of event occurrences.

From Chapter 5.4, we show that it is possible to monitor a subset of PMUs while still preserving event detection. This monitoring scheme involves clustering PMUs based on their electrical distance, relative to the event, possessing a combination of electrically-near 
and electrically-far PMUs. To establish a cluster for the Northwest region of the network, the MFPTV and MTNTV were empirically determined for all PMUs during an event void of any data-related issues. Using a large window size, PMUs with MFPTV > MTNTV were excluded from the cluster as these PMUs would always flag false-positives (data which exceeds the threshold value not related to an event). From here, PMUs with the lowest MFPTVs were selected to be in the cluster as these PMUs have the lowest possibility of flagging false-positives. Only a large window size is considered in this process since $x$ entries will exhibit lower variability compared to small window sizes, corresponding to lower MFPTVs.

In Chapter 5.4, the viability of this monitoring scheme has been demonstrated over three separate instances of a lightning strike occurrences. Clustered PMUs where monitored concurrently with a small and large window size. During events, detection was made within cycles of the lightning strike occurrence with a large window size. The event was confirmed as a power flow contingency, and not a data event, with a small window size. It was noted that detection did not occur among all sites at the same time. From here, voting schemes should be developed to set site-specific event detection flags high when the threshold is exceeded for a specified amount of cycles over a large window size. If a small window size is absent of data related characteristics, this flag should be held high for a predetermined duration of time. If a majority of the cluster flags for an event, perhaps requiring a minimum of one near and one far PMU, then an event is declared.

When analyzing the three separate lightning strike occurrences, the threshold value over 
these three instances varied from each other. Ideally, a single threshold value would be established for each specific PMU in the cluster. This optimal threshold value would minimize or eliminate classifying false-positives while preserving event detection. Furthermore, this value would be used to detect different types of events. To address this, more event-related data needs to be analyzed to determine a suitable threshold value for this particular cluster of PMUs.

To generalize this event detection metric, analysis needs to be performed over events that occur at different locations in the systems. Since only a select few events were analyzed, all of which occur near the same location, the methods described in this thesis may not be applicable to detecting other event types or at other locations. Furthermore, different types of events should be analyzed to validate that this metric can be used for detecting broader types of events. Due to the limited amount of data available in this research area, and the vast amounts of data that monitoring PMUs generate, validating this event detection metric will be a huge challenge moving forward. 


\section{Conclusion}

This research establishes a monitoring framework that aims to facilitate the use of near real-time PMU data streams for decision-making and improved control over modern power systems by employing statistical correlation and statistical distribution parameters. Given the granularity offered by PMUs, the positive sequence voltage phase angles between a pair of PMU locations can be analyzed to fabricate a correlation layer. By mathematically manipulating the correlation vectors to conform to a Rayleigh distribution, pertinent statistical parameters can be quantified and utilized for detecting both power system and data events.

By monitoring these parameters over a combination of PMU data streams, possessing both electrically-near and far data PMUs, a scheme is created for both detection and confirmation of power system events. However, these methods presented here show to be too slow to be utilized at real-time as protection equipment will have already responded to the event disturbance. Due to ease of computation of both correlation and Rayleigh parameters, and coupled with the computational savings gained by monitoring only select PMUs rather than the entire network, this framework may prove to be highly viable for slow and fast cascading event detection.

Prior to deploying these methods for daily operations, further validation must take place on several fronts. First, for the particular location analysis in this work, models need to be developed in which optimal, site-specific K values can be determined. Second, other events 
should be analyzed outside of lightning strike occurrences. Third, events at other locations need to be investigated to ensure this approach to event detection can be generalized. Since only a select few events were analyzed in this work, the results obtained from this analysis may not necessarily be applicable to other event types or at other locations.

To date, the methods presented in this research are suitable for performing analysis over PMU data types; namely negative and zero sequence voltage magnitude and phase angles data, which allows for better detection of unsymmetrical types of events such as doubleline-to-ground and single-line-to-ground faults. In addition, the use of different correlation methods such as sinusoidal or quadratic correlation might result in better coefficients for different power system and data contingencies. These more sophisticated and potentially informative correlation methodologies should be explored to determine if they are more informative.

Analysis can also be expanded to investigate a myriad of events ranging in type and severity. In addition, correlation can be expanded to analyze other characteristics such as the total vector error (see IEEE 37.118), frequency, and rate of change of frequency (ROCOF). 


\section{Bibliography}

[1] T. Pierce Y. Yang and J. Carbonell. A study of retrospective and online event detection. In Proc. 21st ACM Annu. Int. Conf. Res. Develop. Inf. Retrieval, page 28-36, 1998.

[2] Y. Ge, A. J. Flueck, D. K. Kim, J. B. Ahn, J. D. Lee, and D. Y. Kwon. Power system real-time event detection and associated data archival reduction based on synchrophasors. IEEE Transactions on Smart Grid, 6(4):2088-2097, July 2015.

[3] L. Xie, Y. Chen, and P. R. Kumar. Dimensionality reduction of synchrophasor data for early event detection: Linearized analysis. IEEE Transactions on Power Systems, 29(6):2784-2794, Nov 2014.

[4] A. Sant, W. M. Grady, S. Santoso, and J. Ramos. A screening procedure to detect significant power system events recorded by the texas synchrophasor network. In Power and Energy Society General Meeting, 2012 IEEE, pages 1-5, July 2012.

[5] P. M. Ashton, G. A. Taylor, and A. M. Carter. Transient event detection and analysis of the gb transmission system using synchrophasor measurements. In Power Engineering Conference (UPEC), 2013 48th International Universities', pages 1-6, Sept 2013.

[6] S. W. Sohn and A. J. Allen and S. Kulkarni and W. M. Grady and S. Santoso. Event detection method for the PMUs synchrophasor data. In Power Electronics and Machines in Wind Applications (PEMWA), 2012 IEEE, pages 1-7, July 2012.

[7] V. Guralnik and J. Srivastava. Event detection from time series data. In 5th ACM Int. Conf. Knowl. Disc. Data Min., page 33-42, July 1999.

[8] R. Meier, E. Cotilla-Sanchez, B. McCamish, D. Chiu, M. Histand, J. Landford, and R. B. Bass. Power system data management and analysis using synchrophasor data. In Technologies for Sustainability (SusTech), 2014 IEEE Conference on, pages 225-231, July 2014.

[9] B. McCamish, M. Histand, J. Landford, R. B. Bass, D. Chiu, R. Meier, and E. CotillaSanchez. Managing pmu data sets with bitmap indexes. In Technologies for Sustainability (SusTech), 2014 IEEE Conference on, pages 219-224, July 2014.

[10] R. Langner. Stuxnet: Dissecting a cyberwarfare weapon. IEEE Security Privacy, 9(3):49-51, May 2011. 
[11] Jordan Landford, Rich Meier, Richard Barella, Xinghui Zhao, Eduardo Cotilla Sanchez, Robert B. Bass, and Scott A. Wallace. Fast sequence component analysis for attack detection in synchrophasor networks. CoRR, abs/1509.05086, 2015.

[12] K. E. Martin, G. Benmouyal, M. G. Adamiak, M. Begovic, R. O. Burnett, K. R. Carr, A. Cobb, J. A. Kusters, S. H. Horowitz, G. R. Jensen, G. L. Michel, R. J. Murphy, A. G. Phadke, M. S. Sachdev, and J. S. Thorp. Ieee standard for synchrophasors for power systems. IEEE Transactions on Power Delivery, 13(1):73-77, Jan 1998.

[13] IEEE Standard for Synchrophasor Measurements for Power Systems. IEEE Std C37.118.1-2011 (Revision of IEEE Std C37.118-2005), pages 1-61, Dec 2011.

[14] MM. Mukaka. "a guide to appropriate use of correlation coefficient in medical research.". In Malawi Medical Journal : The Journal of Medical Association of Malawi, September 2012.

[15] E. Cotilla-Sanchez, P.D.H. Hines, C. Barrows, S. Blumsack, and M. Patel. MultiAttribute Partitioning of Power Networks Based on Electrical Distance. IEEE Transactions on Power Systems, 28(4):4978-4987, 2013.

[16] Yansong Wang, Jinli Zhao, Fei Zhang, and Binghui Lei. Study on structural vulnerabilities of power grids based on the electrical distance. In Innovative Smart Grid Technologies - Asia (ISGT Asia), 2012 IEEE, pages 1-5, May 2012.

[17] Jong-Bae Park, Ki-Song Lee, Joong-Rin Shin, K.Y. Lee, and D. Chattopadhyay. A new framework of marginal loss factors with consideration of the electrical distance. In Power Engineering Society General Meeting, 2005. IEEE, pages 538-543 Vol. 1, June 2005.

[18] Eric W. Weisstein. Rayleigh distribution. http: / / mathworld.wol fram.com/ RayleighDistribution.html. Accessed: 2016-04-12.

[19] G. Vikas and N. Deepak. n-rayleigh distribution in mobile computing over flatfading channel. In Methods and Models in Computer Science, 2009. ICM2CS 2009. Proceeding of International Conference on, pages 1-3, Dec 2009.

[20] E. E. Kuruoglu and J. Zerubia. Modeling sar images with a generalization of the rayleigh distribution. IEEE Transactions on Image Processing, 13(4):527-533, April 2004.

[21] S. E. Reza, P. Zaman, A. Ahammad, I. Z. Ifty, and M. F. Nayan. A study on data accuracy by comparing between the weibull and rayleigh distribution function to forecast the wind energy potential for several locations of bangladesh. In 2016 th International Conference on the Development in the in Renewable Energy Technology (ICDRET), pages 1-5, Jan 2016. 


\section{Appendix A: Python / MATLAB Code Repository}

Python code can be found at:

https://github.com/benmccamish/BPA

MATLAB code can be found at:

https://github.com/JD4wg/PMU 